\title{
Quark masses using twisted-mass fermion gauge ensembles
}

\author{
C. Alexandrou, ${ }^{1,2}$ S. Bacchio, ${ }^{2}$ G. Bergner, ${ }^{3}$ M. Constantinou, ${ }^{4}$ M. Di Carlo ${ }^{5,6}$ P. Dimopoulos, ${ }^{7}$ J. Finkenrath, ${ }^{2}$ E. Fiorenza, ${ }^{8}$ \\ R. Frezzotti, ${ }^{9}$ M. Garofalo, ${ }^{10}$ K. Hadjiyiannakou, ${ }^{1,2}$ B. Kostrzewa, ${ }^{11}$ G. Koutsou, ${ }^{2}$ K. Jansen, ${ }^{12}$ V. Lubicz ${ }^{13}$ \\ M. Mangin-Brinet,${ }^{14}$ F. Manigrasso, ${ }^{1,9,15}$ G. Martinelli, ${ }^{16}$ E. Papadiofantous, ${ }^{1,2}$ F. Pittler, ${ }^{2}$ G. C. Rossi, ${ }^{9,17}$ \\ F. Sanfilippo, ${ }^{18}$ S. Simula $\odot,{ }^{18}$ C. Tarantino, ${ }^{13}$ A. Todaro,,${ }^{1,915}$ C. Urbach, ${ }^{10}$ and U. Wenger ${ }^{19}$
}

(Extended Twisted Mass Collaboration)

\author{
${ }^{1}$ Department of Physics, University of Cyprus, 20537 Nicosia, Cyprus \\ ${ }^{2}$ Computation-based Science and Technology Research Center, The Cyprus Institute, \\ 20 Konstantinou Kavafi Street, 2121 Nicosia, Cyprus \\ ${ }^{3}$ University of Jena, Institute for Theoretical Physics, Max-Wien-Platz, 1, D-07743 Jena, Germany \\ ${ }^{4}$ Department of Physics, Temple University, Philadelphia, Pennsylvania 19122-1801, USA \\ ${ }^{5}$ School of Physics and Astronomy, The University of Edinburgh, Edinburgh EH9 3FD, United Kingdom \\ ${ }^{6}$ Istituto Nazionale di Fisica Nucleare, Sezione di Roma La Sapienza, \\ Piazzale Aldo Moro 5, I-00185 Roma, Italy \\ ${ }^{7}$ Dipartimento di Scienze Matematiche, Fisiche e Informatiche, Università di Parma and INFN, Gruppo \\ Collegato di Parma, Parco Area delle Scienze 7/a (Campus), 43124 Parma, Italy \\ ${ }^{8} \mathrm{CP}^{3}$-Origins, University of Southern Denmark, Campusvej 55, 5230 Odense, Denmark \\ ${ }^{9}$ Dipartimento di Fisica and INFN, Università di Roma "Tor Vergata", \\ Via della Ricerca Scientifica 1, I-00133 Roma, Italy \\ ${ }^{10}$ HISKP (Theory), Rheinische Friedrich-Wilhelms-Universität Bonn, \\ Nussallee 14-16, 53115 Bonn, Germany \\ ${ }^{11}$ High Performance Computing and Analytics Lab, Rheinische Friedrich-Wilhelms-Universität Bonn, \\ Friedrich-Hirzebruch-Allee 8, 53115 Bonn, Germany \\ ${ }^{12}$ NIC, DESY, Platanenallee 6, D-15738 Zeuthen, Germany \\ ${ }^{13}$ Dipartimento di Matematica e Fisica, Università Roma Tre and INFN, Sezione di Roma Tre, Via della \\ Vasca Navale 84, I-00146 Rome, Italy \\ ${ }^{14}$ Theory Group, Laboratoire de Physique Subatomique et de Cosmologie, \\ CNRS/IN2P3 38026 Grenoble, France \\ ${ }^{15}$ Institut für Physik, Humboldt-Universität zu Berlin, Newtonstrasse 15, 12489 Berlin, Germany \\ ${ }^{16}$ Dipartimento di Fisica and INFN, Università di Roma "La Sapienza", \\ Piazzale Aldo Moro 5, I-00185 Roma, Italy \\ ${ }^{17}$ Centro Fermi, Museo Storico della Fisica e Centro Studi e Ricerche "Enrico Fermi", \\ Piazza del Viminale 1, I-00184 Roma, Italy \\ ${ }^{18}$ Istituto Nazionale di Fisica Nucleare, Sezione di Roma Tre, Via della Vasca Navale 84, \\ I-00146 Rome, Italy \\ ${ }^{19}$ Institute for Theoretical Physics, Albert Einstein Center for Fundamental Physics, University of Bern, \\ Sidlerstrasse 5, CH-3012 Bern, Switzerland
}

(Received 5 May 2021; accepted 15 September 2021; published 19 October 2021)

We present a calculation of the up, down, strange, and charm quark masses performed within the lattice QCD framework. We use the twisted-mass fermion action and carry out simulations that include in the sea two light mass-degenerate quarks, as well as the strange and charm quarks. In the analysis, we use gauge ensembles simulated at three values of the lattice spacing and with light quarks that correspond to pion masses in the range from $350 \mathrm{MeV}$ to the physical value, while the strange and charm quark masses are tuned approximately to their physical values. We use several quantities to set the scale in order to check for finite lattice spacing effects, and in the continuum limit, we get compatible results. The quark mass renormalization is carried out nonperturbatively using the (modified) Regularization Independent Momentum Subtraction (RI'-MOM) method converted into the $\overline{\mathrm{MS}}$ scheme. For the determination of

Published by the American Physical Society under the terms of the Creative Commons Attribution 4.0 International license. Further distribution of this work must maintain attribution to the author(s) and the published article's title, journal citation, and DOI. Funded by $S C O A P^{3}$. 
the quark masses, we use physical observables from both the meson and the baryon sectors, obtaining $m_{u d}=3.636(66)\left(\begin{array}{c}+60 \\ -57\end{array}\right) \mathrm{MeV}$ and $m_{s}=98.7(2.4)\left({ }_{-3.2}^{+4.0}\right) \mathrm{MeV}$ in the $\overline{\mathrm{MS}}(2 \mathrm{GeV})$ scheme and $m_{c}=$ $1036(17)\left(\begin{array}{c}+15 \\ -8\end{array}\right) \mathrm{MeV}$ in the $\overline{\mathrm{MS}}(3 \mathrm{GeV})$ scheme, where the first errors are statistical and the second ones are combinations of systematic errors. For the quark mass ratios, we get $m_{s} / m_{u d}=27.17(32)\left({ }_{-38}^{+56}\right)$ and $m_{c} / m_{s}=11.48(12)\left(\begin{array}{c}+25 \\ -19\end{array}\right)$.

DOI: 10.1103/PhysRevD.104.074515

\section{INTRODUCTION}

Quark masses are essential inputs of the Standard Model (SM) and play a primary role for the description of a large number of physical processes that can provide insights into the dynamics of the SM as well as in the search of beyond the Standard Model physics. The quark masses together with the strong coupling constant can be regarded as the fundamental parameters of QCD, the renormalizable theory of the strong interactions. Therefore, their determination plays a crucial role in the phenomenological description of the plethora of complex phenomena governed by strong nuclear forces taking place in the Universe as well as at particle colliders. Lattice QCD provides a nonperturbative approach based on first principles and systematically improvable for determining the quark masses and the strong coupling. In this approach, the QCD Lagrangian is defined on a discrete Euclidean four-dimensional spacetime lattice of large but finite extent, which allows for numerical simulation of the theory via Monte Carlo methods. The finite volume and the nonvanishing lattice spacing introduce systematic artifacts, which can be theoretically understood, kept under numerical control, and extrapolated away in order to extract the physical quantities of interest.

Theoretical progress in lattice field theory and improvement in numerical algorithms, accompanied with a continuously increasing computational power, are allowing us to perform simulations using physical values of the light-quark masses. However, most of these simulations are still carried out using a single lattice spacing and volume, although this is rapidly changing as more lattice QCD collaborations gain access to larger computational resources and can produce multiple ensembles of gauge configurations generated with physical values of the light quark masses. Such ensembles will be referred to as physical point ensembles. In this work, we include two physical point ensembles at two different lattice spacings. In order to take the continuum limit, we also employ additional ensembles at a coarser lattice resolution with larger than physical pion masses. Globally, we thus use ensembles with three values of the lattice spacing and spanning pion masses in the range from about 350 to $135 \mathrm{MeV}$, which enable us to perform a combined chiral and continuum extrapolation. In order to study systematic effects in the determination of the quark masses, we use two sets of observables to set the scale and to evaluate the quark masses. One set of observables is based on quantities from the meson sector of QCD, while the other set relies on baryonic observables. In the former case, we use the pion mass and decay constant to set the scale and to determine the average up/down quark mass. The mass of the strange and charm quarks are extracted using the kaon and $D$-meson masses, respectively. In the latter case, instead, the masses of the pion and nucleon are employed to set the scale and fix the average up/down quark mass, while the $\Omega^{-}$and the $\Lambda_{c}$ masses determine the strange and charm quark masses, respectively. In this way, we obtain a valuable consistency check with respect to the results coming from the mesonic sector.

For the renormalization of the quark mass, we employ a dedicated set of gauge ensembles with four massdegenerate sea quarks (having mass around half of the strange quark mass). Such a set ensures a good control of the extrapolation to the massless limit. We perform the computation in an intermediate mass-independent scheme, which is finally converted to the standard $\overline{\mathrm{MS}}$ scheme.

The paper is organized as follows. In Sec. II, we describe the gauge ensembles used in this study and explain our methodology. In Secs. III and IV, we present the methods used to set the lattice spacing $a$ and to carry out a nonperturbative computation of the renormalization constant $Z_{P}$ including a detailed discussion on the control of hadronic contaminations and other systematic errors. In Secs. V and VI, we describe the extraction of the quark masses and their ratios using inputs from the mesonic and baryonic sectors, respectively. In Sec. VII, we discuss our final results and give our conclusions and outlook.

\section{METHODOLOGY}

In twisted-mass lattice QCD [1], the discretized Dirac operator in the physical quark basis is written as

$$
\begin{aligned}
D\left(\mu_{f}\right)= & \frac{1}{2} \gamma_{\mu}\left(\nabla_{\mu}+\nabla_{\mu}^{*}\right)-i \gamma_{5}\left\{m_{\mathrm{cr}}\left(r_{f}\right)-r_{f} \frac{a}{2} \nabla_{\mu} \nabla_{\mu}^{*}\right. \\
& \left.+\frac{c_{S W}\left(r_{f}\right)}{32} \gamma_{\mu} \gamma_{\nu}\left(Q_{\mu \nu}-Q_{\nu \mu}\right)\right\}+\mu_{f},
\end{aligned}
$$

where $r_{f}= \pm 1, \nabla_{\mu}$ and $\nabla_{\mu}^{*}$ are nearest-neighbor forward and backward covariant derivatives, $\mu_{f}$ sets the mass of the quark field $q_{f}$ of flavor $f, c_{S W}$ is the coefficient of the clover-term $Q_{\mu \nu}$ [2], and $m_{c r}$ is the critical value of the "untwisted" mass $m_{0}$, obtained by requiring the vanishing of the partially conserved axial current (PCAC) mass, as 
discussed in Ref. [3]. This condition, referred to as maximal twist, guarantees automatic $\mathcal{O}(a)$ improvement of physical observables [4,5]. In the twisted-mass fermion formulation at maximal twist, the renormalized quark masses are thus given by

$$
m_{f}=\frac{\mu_{f}}{Z_{P}}=\frac{\left(a \mu_{f}\right)}{a Z_{P}}
$$

where $a$ is the lattice spacing and $Z_{P}$ is the pseudoscalar renormalization constant. Therefore, the determination of both $Z_{P}$ and the lattice spacing, combined with inputs from known physical quantities depending on the quark masses, enables us to extract $m_{f}$. Having gauge ensembles with at least three different lattice spacings at several pion masses allows us to take the continuum limit, and by performing a chiral extrapolation, we can determine the quark masses at the physical point.

\section{A. Gauge ensembles}

We use the twisted-mass fermion discretization scheme [1,4] with the inclusion of a clover term [2]. As already explained, twisted-mass fermions (TMFs) provide an attractive formulation for lattice QCD simulations allowing for automatic $\mathcal{O}(a)$ improvement of physical observables as well as renormalization constants $[4,6]$. This is an important property since quantities of interest have lattice artifacts of $\mathcal{O}\left(a^{2}\right)$ and are thus closer to the continuum limit. A clover term is added to the TMF action to suppress
$\mathcal{O}\left(a^{2}\right)$ breaking effects between the neutral and charged pions, which eventually leads to the stabilization of simulations with light quark masses close to the physical pion mass. For more details on the TMF formulation, see Refs. [7,8], and on the simulation and tuning strategies, see Refs. $[9,10]$.

In this study, we analyze ten gauge ensembles simulated at three values of the lattice spacing and at several values of the pion mass, spanning a range from the physical pion mass up to $350 \mathrm{MeV}$. Some parameters of these ensembles and the values of few key physical quantities are listed in Table I. More details are given in Ref. [11]. With respect to Ref. [11], the ensemble cC211.20.48 has been added in order to investigate the light quark mass dependence at the finest lattice spacing.

The ensembles are generated with two mass-degenerate light quarks and the strange and charm quarks in the sea $\left(N_{f}=2+1+1\right.$ ensembles). The strange and charm sea quark mass parameters, $a \mu_{\sigma}$ and $a \mu_{\delta}$ (see, e.g., Eq. (8) of Ref. [12]) have been adjusted so as to reproduce the phenomenological conditions $m_{c} / m_{s} \simeq 11.8$ and $m_{D_{s}} / f_{D_{s}} \simeq$ 7.9 [13], which are easy to implement with few percent level precision even using simulations with larger than physical pion masses and on lattices of linear size $L \simeq 2.5 \mathrm{fm}$, as detailed in Ref. [10]. The condition on $m_{D_{s}} / f_{D_{s}}$ is sensitive to the charm quark mass, while the one on $m_{c} / m_{s}$ fixes the strange quark mass. In this way, the charm and strange sea quark mass parameters have been tuned, separately for each lattice resolution (or $\beta$ ), to bare values that a posteriori turn out to yield values for the renormalized sea quark masses that

TABLE I. Parameters of the $N_{f}=2+1+1$ ensembles analyzed in this study. In the first column, we give the name of the ensemble; in the second, we give the lattice volume; in the third, we give the number of molecular dynamics units simulated per ensemble; in the fourth, we give the twisted-mass parameter, $a \mu_{\ell}$, for the average up/down (light) quark; in the fifth and in the sixth, we give the pion mass $a m_{\pi}$ and decay constant $a f_{\pi}$ in lattice units from Ref. [11]; in the seventh, we give the pion mass times the lattice spatial length, $m_{\pi} L$; in the eighth, we give the ratio $m_{N} / m_{\pi}$ as determined in Sec. VI; and, finally, in the last column, we give the pion mass in physical units, using our determination of the gradient-flow scale $w_{0}$ obtained in Ref. [11] [see later Eq. (32)]. We also include for each set of ensembles with the same lattice spacing the coupling constant $\beta$, the clover-term parameter $c_{S W}$, the parameters of the nondegenerate operator $a \mu_{\sigma}$ and $a \mu_{\delta}$, related to the renormalized strange and charm sea quark masses [5], and the value of the gradient-flow scale $w_{0} / a$ determined at the physical pion mass in Ref. [11].

\begin{tabular}{|c|c|c|c|c|c|c|c|c|}
\hline Ensemble & $L^{3} \times T$ & MDUs & $a \mu_{\ell}$ & $a m_{\pi}$ & $a f_{\pi}$ & $m_{\pi} L$ & $m_{N} / m_{\pi}$ & $m_{\pi}(\mathrm{MeV})$ \\
\hline \multicolumn{9}{|c|}{$\beta=1.726, c_{S W}=1.74, a \mu_{\sigma}=0.1408, a \mu_{\delta}=0.1521, w_{0} / a=1.8352(35)$} \\
\hline cA211.53.24 & $24^{3} \times 48$ & 5026 & 0.00530 & $0.16626(51)$ & $0.07106(36)$ & 3.99 & $\ldots$ & $346.4(1.6)$ \\
\hline cA211.40.24 & $24^{3} \times 48$ & 5298 & 0.00400 & $0.14477(70)$ & $0.06809(30)$ & 3.47 & $\ldots$ & $301.6(2.1)$ \\
\hline cA211.30.32 & $32^{3} \times 64$ & 10234 & 0.00300 & $0.12530(16)$ & $0.06674(15)$ & 4.01 & $4.049(14)$ & $261.1(1.1)$ \\
\hline cA211.12.48 & $48^{3} \times 96$ & 2936 & 0.00120 & $0.08022(18)$ & $0.06133(33)$ & 3.85 & $5.685(28)$ & $167.1(0.8)$ \\
\hline \multicolumn{9}{|c|}{$\beta=1.778, c_{S W}=1.69, a \mu_{\sigma}=0.1246864, a \mu_{\delta}=0.1315052, w_{0} / a=2.1299(16)$} \\
\hline cB211.25.32 & $32^{3} \times 64$ & 3959 & 0.00250 & $0.10475(45)$ & $0.05652(38)$ & 3.35 & $4.104(36)$ & $253.3(1.4)$ \\
\hline cB211.25.48 & $48^{3} \times 96$ & 5246 & 0.00250 & 0.10465 (14) & $0.05726(12)$ & 5.02 & $4.124(17)$ & 253.0 \\
\hline cB211.14.64 & $64^{3} \times 128$ & 6187 & 0.00140 & $0.07848(10)$ & $0.05477(12)$ & 5.02 & $5.119(36)$ & $189.8(0.7)$ \\
\hline cB211.072.64 & $64^{3} \times 128$ & 3161 & 0.00072 & $0.05659(8)$ & $0.05267(14)$ & 3.62 & $6.760(30)$ & $136.8(0.6)$ \\
\hline \multicolumn{9}{|c|}{$\beta=1.836, c_{S W}=1.6452, a \mu_{\sigma}=0.106586, a \mu_{\delta}=0.107146, w_{0} / a=2.5045(17)$} \\
\hline cC211.20.48 & $48^{3} \times 96$ & 2000 & 0.00200 & $0.08540(17)$ & $0.04892(13)$ & 4.13 & $4.244(25)$ & $245.73(98)$ \\
\hline $\mathrm{cC} 211.06 .80$ & $80^{3} \times 160$ & 3207 & 0.00060 & $0.04720(7)$ & $0.04504(10)$ & 3.78 & $6.916(19)$ & $134.3(0.5)$ \\
\hline
\end{tabular}


are consistent within statistical errors of few percents with those we determine in this paper, as discussed in the following, at the physical pion mass point, on large volumes and in the continuum limit.

\section{B. Osterwalder-Seiler fermions}

A naive use of the twisted-mass action for nondegenerate strange and charm quarks would lead to an undesired $\mathrm{O}\left(a^{2}\right)$ mixing of the strange and charm flavors in the correlation functions of interest to determine physical quantities $[14,15]$. In order to avoid such a mixing in the correlation functions, we adopt a nonunitary lattice setup [5] where the twistedmass action for nondegenerate strange and charm quarks is employed only in the sea sector, while the valence strange and charm quarks that enter the correlation functions are regularized as exactly flavor-diagonal Osterwalder-Seiler fermions [16]. Thus, the valence action in the strange and charm sectors $(f=s, c)$ reads

$$
S_{O S}^{f}\left(\mu_{f}\right)=a^{4} \sum_{x} \bar{q}_{f}(x) D\left(\mu_{f}\right) q_{f}(x),
$$

where $D\left(\mu_{f}\right)$ is the twisted-mass Dirac operator in Eq. (1) with the same values of $m_{0}$ and $c_{S W}$ as in the sea sector action used for ensemble generations. As the renormalized strange and charm sea quark masses are matched with few percent relative accuracy to their valence counterparts, no significant unitarity violation is expected in our continuum limit results.

When constructing meson correlation functions, the Wilson parameters of the two valence quarks are always chosen to have opposite values. This choice guarantees that squared pseudoscalar meson masses, generically indicated by $m_{\mathrm{PS}}^{2}$, differ from their continuum counterparts only by terms of order $\mathcal{O}\left(a^{2} \mu\right)$ [4,7]. As we said above, in our lattice setup, the (valence) flavor conservation is guaranteed in all correlation functions, and automatic $\mathcal{O}(a)$ improvement is maintained. Of course, we need to fix the valence strange and charm quark masses, $\mu_{s}$ and $\mu_{c}$, by imposing suitable renormalization conditions. For this purpose, in the present work, we use two different sets of observables. Namely, in one case, we use the mass of the physical masses of kaon and $D$ (or $D_{s}$ ) mesons, and in the other case, we use the masses of the $\Omega$ and $\Lambda_{c}$ baryons. These two different choices will lead to two different determinations of the strange and charm quark masses, which will enable us to check the consistency of our results when using physical inputs from the mesonic and baryonic sectors.

\section{SCALE SETTING}

As already mentioned, we have ensembles at three different lattice spacings. We will refer to the ensembles in Table I that start with $c A$ in their names as A ensembles, those starting with $c B$ as B ensembles, and those with $c C$ as
TABLE II. The values of the lattice spacing extracted from the pion sector (second row) and using the nucleon mass (third row) for the $\mathrm{A}, \mathrm{B}$, and $\mathrm{C}$ ensembles, denoted by $a_{A}, a_{B}$, and $a_{C}$, respectively. In the last row, we show the difference $\Delta a$ between the lattice spacings determined using the nucleon mass and from the pion sector.

\begin{tabular}{lccc}
\hline \hline Sector & $a_{A}(\mathrm{fm})$ & $a_{B}(\mathrm{fm})$ & $a_{C}(\mathrm{fm})$ \\
\hline Pion & $0.09471(39)$ & $0.08161(30)$ & $0.06942(26)$ \\
Nucleon & $0.09295(47)$ & $0.07975(32)$ & $0.06860(20)$ \\
$\Delta a$ & $0.00176(61)$ & $0.00186(44)$ & $0.00082(32)$ \\
\hline \hline
\end{tabular}

C ensembles (the label $c$ stands for clover). Each of these groups has the same lattice spacing, with the A ensembles having the largest lattice spacing and $\mathrm{C}$ ensembles having the smallest one. In what follows, we will use different quantities to determine the three lattice spacings. This will allow us to check consistency while taking the continuum limit when different inputs are used. In the pion sector, the pion mass and decay constant are used as input. Within this approach, one also determines the value of the gradient-flow scale $w_{0}$. We use the isosymmetric values of the pion mass and decay constant, given, respectively, by [17]

$m_{\pi}^{\text {isoQCD }}=135.0(2) \mathrm{MeV}$ and $f_{\pi}^{\text {isoQCD }}=130.4(2) \mathrm{MeV}$.

We also compute the value of $w_{0} / a$ for each ensemble (see Table I) and extrapolate to the physical pion mass and continuum limit. We find $w_{0}=0.17383(63) \mathrm{fm}$ [11], and using this value, one determines the lattice spacings shown in Table II. We refer to this determination of the lattice spacings as coming from the "pion" sector. Details are given in Ref. [11].

Another quantity used for the determination of the lattice spacings is the mass of the nucleon [18,19]. Details on the extraction of the nucleon mass are given in Sec. VI. In order to fit the pion mass dependence of the nucleon mass, we use the well established $\mathrm{SU}(2)$ chiral perturbation theory result to one loop [20,21],

$$
m_{N}\left(m_{\pi}\right)=m_{N}^{0}-4 c_{1} m_{\pi}^{2}-\frac{3 g_{A}^{2}}{16 \pi f_{\pi}^{2}} m_{\pi}^{3} .
$$

The three values of the lattice spacing, which will be denoted by $a_{A}, a_{B}$, and $a_{C}$, can be determined from the lattice data for the nucleon and pion masses by rewriting Eq. (5) as

$$
\left(a_{i} m_{N}\right)=a_{i} m_{N}^{0}-4 c_{1} \frac{\left(a_{i} m_{\pi}\right)^{2}}{a_{i}}-\frac{3 g_{A}^{2}}{16 \pi f_{\pi}^{2}} \frac{\left(a_{i} m_{\pi}\right)^{3}}{a_{i}^{2}},
$$



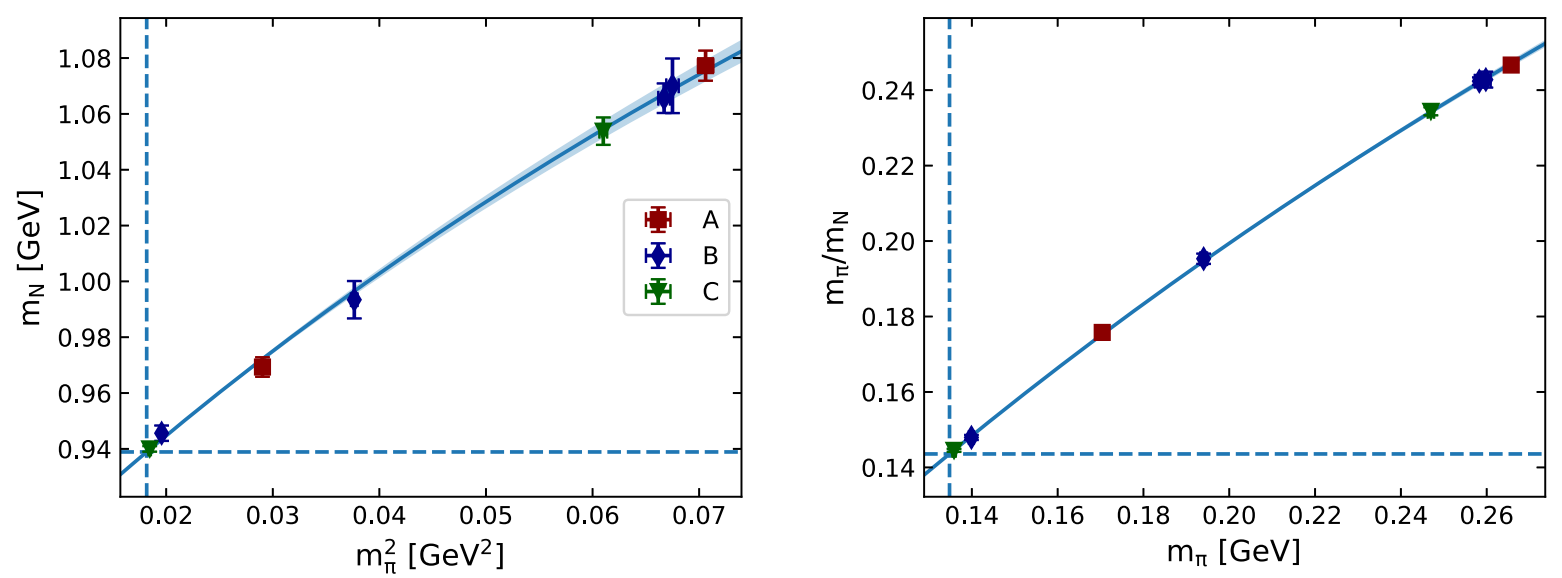

FIG. 1. Determination of the lattice spacing from the nucleon mass. In the left panel, we show the nucleon mass as a function of the pion mass $m_{\pi}$ squared. In the right panel, we show the dimensionless quantity $m_{\pi} / m_{N}$ as a function of $m_{\pi}$, using the lattice spacing extracted from the nucleon mass. The values of $m_{\pi} / m_{N}$ are listed in Table I, while $a m_{N}$ and $a m_{\pi}$ are determined in Sec. VI. The solid line shows the fit to the lattice QCD data using Eq. (5). The value of $\chi^{2} /$ d.o.f. is 0.19 , where the number of degrees of freedom is 5 .

where $\left(a_{i} m_{N}\right)$ and $\left(a_{i} m_{\pi}\right)$ are our lattice QCD results and $i=A, B, C$. The three quantities $a_{i}$ as well as the nucleon mass in the chiral limit, $m_{N}^{0}$, are treated as fitting parameters, while the value of $c_{1}$ is fixed by requiring the reproduction of the physical value of the nucleon mass, $m_{N}^{\text {isoQCD }}$, at the physical pion point (4), namely,

$c_{1}=\left[m_{N}^{0}-\frac{3 g_{A}^{2}}{16 \pi f_{\pi}^{2}}\left(m_{\pi}^{\mathrm{isoQCD}}\right)^{3}-m_{N}^{\mathrm{isoQCD}}\right] /\left[4\left(m_{\pi}^{\mathrm{isoQCD}}\right)^{2}\right]$.

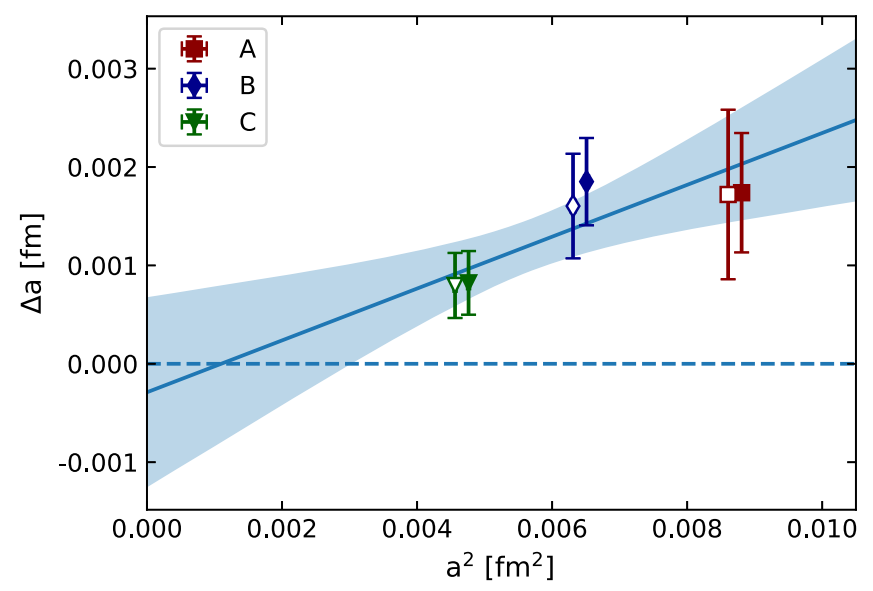

FIG. 2. The difference $\Delta a$ between the lattice spacings determined from the pion sector and the nucleon mass versus $a^{2}$. Full symbols are the lattice spacings determined using all the ensembles for which $m_{\pi}<260 \mathrm{MeV}$. Open symbols, shifted to the left for clarity, are obtained using ensembles for which the pion mass is below $190 \mathrm{MeV}$. The solid line shows the linear fit in $a^{2}$ to the results extracted by using ensembles with $m_{\pi}<$ $260 \mathrm{MeV}$ (full symbols), which is largely consistent with zero in the continuum limit.
We restrict ourselves to using ensembles for which the pion mass is below $260 \mathrm{MeV}$ since chiral perturbation theory to higher orders has larger ambiguities. The simulations of the gauge ensembles use mass-degenerate up and down quarks and include no electromagnetic effects. Thus, we use the average value of the proton and neutron mass as our input for fixing the lattice spacings; namely, we assume $m_{N}^{\text {isoQCD }}=0.9389 \mathrm{GeV}$ in Eq. (7). We also use the physical value for the axial charge, $g_{A}=1.27641$ (56) [22], and for consistency the physical value of $f_{\pi}$ from Eq. (4). The ratio $g_{A} / f_{\pi}$ appears in the $m_{\pi}^{3}$ term, and any residual correction due to strong isospin breaking and electromagnetism is neglected.

The result of the fit to the mass of the nucleon $m_{N}$ is depicted in Fig. 1 and describes very well the data, yielding $\chi^{2} /$ d.o.f. $=0.19$, where d.o.f. are the number of degrees of freedom. We find $m_{N}^{0}=0.8737(14) \mathrm{GeV}$, $c_{1}=-1.090(20) \mathrm{GeV}^{-1}$, and the values of the lattice spacings shown in the third row of Table II. In Fig. 1, we also show the ratio $m_{N} / m_{\pi}$ and the resulting fit using the parameters extracted from the fit to the nucleon mass. As can be seen, the data for $m_{N} / m_{\pi}$ are well described.

The values of the lattice spacing extracted from the pion sector and from the nucleon mass are shown in Table II, and they differ by $\mathcal{O}\left(a^{2}\right)$ effects. Fitting their difference as a function of $a^{2}$ is shown in Fig. 2. We observe that in the continuum limit the difference vanishes, as expected for our $\mathcal{O}(a)$-improved formalism. In what follows, we will use both determinations to extract the quark masses. This provides a cross-check for our procedure and for the magnitude of any residual lattice spacing effect.

\section{COMPUTATION OF $Z_{P}$}

In order to determine the renormalized quark masses, it is crucial to perform an accurate evaluation of the mass 
TABLE III. Parameters for the $N_{f}=4$ ensembles used for the calculation of $Z_{P}$. By $a m_{\mathrm{PS}}$, we denote the pseudoscalar (nonsinglet) meson mass, and by $a m_{\mathrm{PCAC}}$, we denote the PCAC untwisted mass (see Ref. [3]), both given in lattice units. Note that on all the $N_{f}=4$ ensembles, taking into account statistical errors, the values of $a m_{\mathrm{PCAC}}$ are typically 15 to 100 times smaller than the lowest twisted-mass values.

\begin{tabular}{|c|c|c|c|c|c|c|c|c|}
\hline \multicolumn{3}{|c|}{$\beta=1.72624^{3} \times 48$} & \multicolumn{3}{|c|}{$\beta=1.77824^{3} \times 48$} & \multicolumn{3}{|c|}{$\beta=1.83632^{3} \times 64$} \\
\hline$a \mu_{\text {sea }}$ & $a m_{\mathrm{PS}}$ & $a m_{\text {PCAC }}$ & $a \mu_{\text {sea }}$ & $a m_{\mathrm{PS}}$ & $a m_{\mathrm{PCAC}}$ & $a \mu_{\text {sea }}$ & $a m_{\mathrm{PS}}$ & $a m_{\mathrm{PCAC}}$ \\
\hline 0.0060 & $0.1689(15)$ & $-4.1(1.4) \times 10^{-4}$ & 0.0075 & $0.1748(15)$ & $-2.3(0.8) \times 10^{-5}$ & 0.0050 & $0.1276(14)$ & $-4.3(3.1) \times 10^{-5}$ \\
\hline 0.0080 & $0.1905(11)$ & $-4.3(1.1) \times 10^{-5}$ & 0.0088 & $0.1871(18)$ & $-8.6(8.0) \times 10^{-5}$ & 0.0065 & $0.1447(14)$ & $+5.9(2.1) \times 10^{-5}$ \\
\hline 0.0100 & $0.2155(12)$ & $+1.5(1.3) \times 10^{-4}$ & 0.0100 & $0.2006(18)$ & $-1.6(0.8) \times 10^{-4}$ & 0.0080 & $0.1585(14)$ & $+1.6(0.3) \times 10^{-4}$ \\
\hline 0.0115 & $0.2289(12)$ & $+1.7(1.1) \times 10^{-4}$ & 0.0115 & $0.2158(11)$ & $+0.2(9.5) \times 10^{-5}$ & 0.0095 & $0.1744(12)$ & $+2.0(0.3) \times 10^{-4}$ \\
\hline
\end{tabular}

renormalization factor $Z_{m}$, that in the maximally twistedmass formulation is given by $Z_{m}=1 / Z_{P}$ [see Eq. (2)]. For this reason, the details of the procedure we have followed to compute $Z_{P}$ will be given in this section.

For the calculation of $Z_{P}$, we employ the nonperturbative $\mathrm{RI}^{\prime}$-MOM renormalization scheme [23], which is a massindependent scheme since the renormalization constants are defined in the massless limit. The choice of this intermediate scheme is convenient in that the scale evolution for the renormalization constants of the operators with nontrivial anomalous dimension is controlled by the renormalized gauge coupling alone. This requires, however, simulations close enough to the chiral limit, which is not the case of the $N_{f}=2+1+1$ ensembles of Table I, mainly due to the presence of the heavy sea charm quark. In order to safely take the chiral limit in the computation of the renormalization constants, we have thus separately produced gauge field configurations with four mass-degenerate quarks $\left(N_{f}=4\right)$ at the same value of the coupling $\beta$ and with the same clover term included in the fermionic action as for our A, B, and C ensembles of Table I. This ensures that in the chiral limit the same massless $N_{f}=4$ QCD theory underlies both the ensembles used for computing hadronic observables and setting the energy scale (see Table I) and the ensembles dedicated to the evaluation of the renormalization constants, about which details are given in Table III. The four degenerate quarks are taken with masses from approximately 8 to 16 times larger than the average up-down quark mass, which simplifies both the simulations and the tuning to maximal twist. The values of the critical mass $m_{c r}$ have been chosen in order to satisfy the maximal twist condition (which is convenient to reduce lattice artifacts) to a very good accuracy level, as can be deduced from the smallness of the PCAC masses in Table III (actually, the tuning is even slightly better than the one corresponding to the $N_{f}=2+1+1$ ensembles of Table I).

In the $\mathrm{RI}^{\prime}$-MOM scheme, we obtain the renormalization constant of the (flavor nonsinglet) pseudoscalar density operator $O_{P}=i \bar{q} \gamma_{5} q$ with $r_{q}^{\prime}=-r_{q}=-1$ and $\mu_{q^{\prime}}=\mu_{q}$ via the following condition, ${ }^{1}$

$$
\left.Z_{q}^{-1} Z_{P} \frac{1}{12} \operatorname{Tr}\left[\gamma^{5} \mathcal{V}_{P}(p)\right]\right|_{p^{2}=\mu_{0}^{2}}=1
$$

where $\mathcal{V}_{P}$ is the pseudoscalar vertex function between quark and antiquark states with momentum $p$ and $Z_{q}$ is the renormalization constant of the quark field, defined as

$$
Z_{q}=-\left.\frac{i}{12} \operatorname{Tr}\left[\frac{\not p}{p^{2}} S_{q}^{-1}(p)\right]\right|_{p^{2}=\mu_{0}^{2}} .
$$

Here, $S_{q}$ is the quark propagator at momentum $p$, which is identified with the renormalization scale $\mu_{0}$. In this work, we adopt the alternative definition of $Z_{q}$ first proposed in Ref. [6],

$$
Z_{q}=-\left.\frac{i}{12 N_{p}} \sum_{\mu}^{\prime} \operatorname{Tr}\left[\frac{\gamma_{\mu}}{\tilde{p}_{\mu}} S_{q}^{-1}(p)\right]\right|_{p^{2}=\mu_{0}^{2}},
$$

where the sum $\sum^{\prime}$ is over the $N_{p}$ nonvanishing components of the lattice momentum $a \tilde{p}_{\mu}=\sin \left(a p_{\mu}\right)$. The prescription of Eq. (10), unlike the naive RI'-MOM definition (9), has no lattice artifacts at tree level, and beyond tree level, it exhibits quite small $\mathcal{O}\left(a^{2}\right)$ cutoff effects.

The subtraction of the Goldstone pole in the vertex function $\mathcal{V}_{P}$ requires good control of the vertex mass dependence. Therefore, we find it more suitable to adopt a partially quenched (PQ) setup, in which propagators and vertices are computed for multiple values of valence quark masses $\mu_{\mathrm{val}}$ at fixed sea mass $\mu_{\text {sea }}$; namely, we use

\footnotetext{
${ }^{1}$ The relevant fermionic action density is $\bar{q} D\left(\mu_{q}\right) q+$ $\bar{q}^{\prime} D\left(\mu_{q^{\prime}}\right) q^{\prime}=\bar{X}\left[\gamma_{\nu}\left(\nabla+\nabla^{*}\right)_{\nu} / 2+\left(m_{c r}(1)-a / 2 \nabla_{\nu}^{*} \nabla_{\nu}\right)+i \gamma_{5} \tau^{3} \mu_{q}+\right.$ $\left.\left(c_{s w}(1) / 32\right) \gamma_{\mu} \gamma_{\nu}\left(Q_{\mu \nu}-Q_{\nu \mu}\right)\right] X$, where $X=\left(\chi_{q}, \chi_{q^{\prime}}\right)^{T}$ is a valence quark field pair in the canonical quark basis for standard Wilson fermions (after which, as usual, the renormalization constants are named) and $q=\exp \left(i \gamma_{5} \pi / 4\right) \chi_{q}, \quad q^{\prime}=\exp \left(-i \gamma_{5} \pi / 4\right) \chi_{q^{\prime}}$, $\bar{q}=\bar{\chi}_{q} \exp \left(i \gamma_{5} \pi / 4\right)$, and $\bar{q}^{\prime}=\bar{\chi}_{q^{\prime}} \exp \left(-i \gamma_{5} \pi / 4\right)$. Note in particular that $i \bar{q} \gamma_{5} q^{\prime}=i \bar{\chi}_{q} \gamma_{5} \chi_{q^{\prime}}$.
} 


$$
\begin{aligned}
& a \mu_{\mathrm{val}}^{\mathrm{A}}=\{0.0060,0.0080,0.0100,0.0115,0.0130,0.0150,0.0170,0.0190,0.0210\}, \\
& a \mu_{\mathrm{val}}^{\mathrm{B}}=\{0.0050,0.0060,0.0075,0.0090,0.0100,0.0110,0.0130,0.0150,0.0170\}, \\
& a \mu_{\mathrm{val}}^{\mathrm{C}}=\{0.0040,0.0050,0.0065,0.0080,0.0095,0.0110,0.0125,0.0140,0.0155\} .
\end{aligned}
$$

The chiral extrapolation of the pseudoscalar vertex is discussed in details in Sec. IV B below.

For each ensemble, we employ a large number of momenta $a p=2 \pi\left(\frac{n_{t}}{T}, \frac{n_{x}}{L}, \frac{n_{y}}{L}, \frac{n_{z}}{L}\right)$, in the range $(a p)^{2} \in$ $[0.24,6.69]$ for the $24^{3} \times 48$ ensembles and $(a p)^{2} \in$ $[0.13,5.23]$ for the $32^{3} \times 64$ ensembles. Quark propagators and vertices in momentum space are evaluated using $N_{\text {cfg }}=200$ gauge configurations. In order to reduce the effect of Lorentz noninvariant cutoff effects, we filter the momenta selecting the ones that are isotropic ("democratic") in the spatial directions, thus satisfying

$$
\frac{\sum_{\mu} \tilde{p}_{\mu}^{4}}{\left(\sum_{\mu} \tilde{p}_{\mu}^{2}\right)^{2}}<0.28 .
$$

The above constraint ensures that unwanted hypercubic lattice artifacts are suppressed [6]. We further improve the $Z_{P}$ estimator by using results from lattice perturbation theory (for more details see, e.g., Refs. [24,25]). In summary, we calculate the lattice artifacts at one-loop level and to all orders in the lattice spacing, $\mathcal{O}\left(g^{2} a^{\infty}\right)$. The perturbative corrections to the Green's function of the pseudoscalar operator, as well as to the quark propagator, are evaluated for each momentum $a p$ at which the renormalization constants are computed. It should be noted that each value of ap requires a separate calculation of the $\mathcal{O}\left(g^{2} a^{\infty}\right)$ correction, as the perturbative contributions are not analytical and require the numerical evaluation of oneloop integrals. Such contributions also include the leadingorder terms, $\mathcal{O}\left(g^{2} a^{0}\right)$, that have to be separated from the pure $\mathcal{O}\left(a^{n}\right)$ terms $(n \neq 0)$. The computation of perturbative lattice artifacts to $\mathcal{O}\left(g^{2} a^{\infty}\right)$ done in Ref. [25] is adapted for the case of the specific definition of $Z_{q}$ in Eq. (10). Thus, the one-loop perturbative corrections that we use are defined as

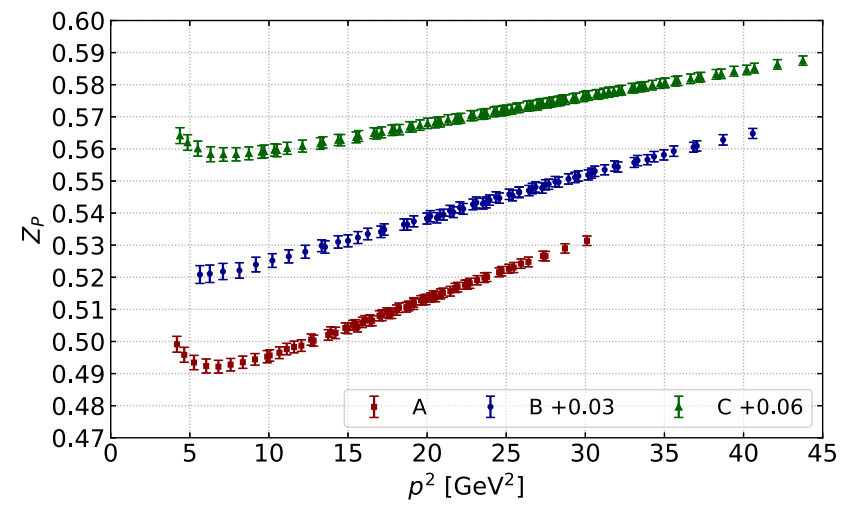

$\Delta Z_{q}^{(1)}(a p)=Z_{q}^{(1)}(\log (a p), a p)-Z_{q}^{(1)}(\log (a p), 0)$,

$\Delta \mathcal{V}_{P}^{(1)}(a p)=\mathcal{V}_{P}^{(1)}(\log (a p), a p)-\mathcal{V}_{P}^{(1)}(\log (a p), 0)$,

where $Z_{q}^{(1)}$ and $\mathcal{V}_{P}^{(1)}$ are the one-loop contributions to the quark field renormalization constant and to the pseudoscalar vertex, respectively.

Using the above quantities, we extract improved nonperturbative estimates for $Z_{q}$ and $Z_{P}$ by modifying the renormalization conditions as

$$
\begin{gathered}
Z_{q}^{\mathrm{impr}}=Z_{q}-g^{2} \Delta Z_{q}^{(1)}\left(a \mu_{0}\right), \\
\left.\left(Z_{q}^{\mathrm{impr}}\right)^{-1} Z_{P}^{\mathrm{impr}} \frac{1}{12} \operatorname{Tr}\left[\gamma^{5}\left(\mathcal{V}_{P}-g^{2} \Delta \mathcal{V}_{P}^{(1)}(a p)\right)\right]\right|_{p^{2}=\mu_{0}^{2}}=1 .
\end{gathered}
$$

\section{A. Analysis method and safety checks against hadronic contaminations}

The improved $Z_{P}$ estimators, namely, $Z_{P}^{\mathrm{impr}}$ obtained from Eq. (16), are evaluated at different values of $p^{2}=\mu_{0}^{2}$ and extrapolated to the chiral limit, a step which is discussed in detail in the following Sec. IV B. Then, the chirally extrapolated lattice estimators of $Z_{P}$ are evolved to a common reference scale $p^{2}=\mu_{\text {ref }}^{2}$ in the $\mathrm{RI}^{\prime}$-MOM scheme using the anomalous dimension known up to next-to-next-to-next leading order $\left(\mathrm{N}^{3} \mathrm{LO}\right)$ according to Ref. [26] and adopting $\Lambda_{\mathrm{QCD}}\left(N_{f}=4\right)=294(12) \mathrm{MeV}$ from Ref. [13]. The curves obtained at the three $\beta$ values are reported in Fig. 3 for two different values of the reference scale, namely, $\mu_{\text {ref }}^{2}=$ $17 \mathrm{GeV}^{2}$ and $\mu_{\text {ref }}^{2}=21 \mathrm{GeV}^{2}$.

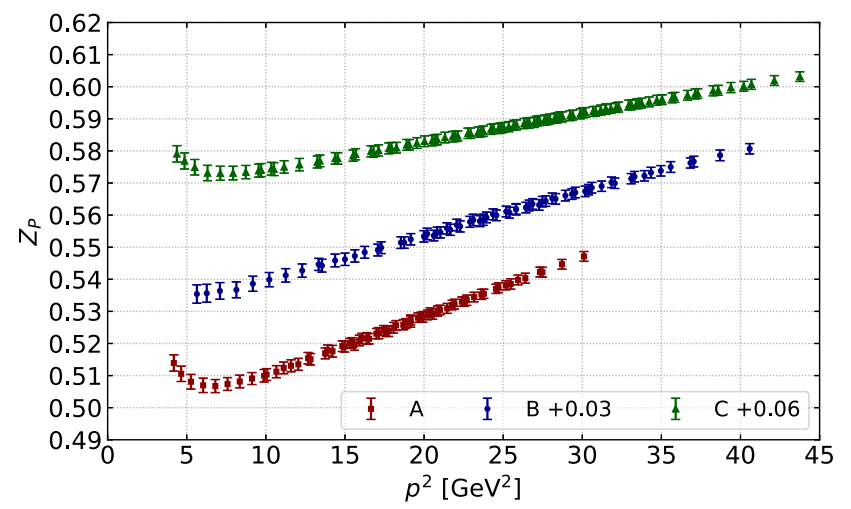

FIG. 3. Dependence of $Z_{P}$ on the momentum $p^{2}$ for two values of the reference scale, $\mu_{\text {ref }}^{2}=17 \mathrm{GeV}^{2}$ (left panel) and $\mu_{\text {ref }}^{2}=21 \mathrm{GeV}^{2}$ (right panel). The vertical offsets shown in the inset are applied to the data of the corresponding ensembles. 
TABLE IV. Results of the two fits on $Z_{P}\left(\mu_{\mathrm{ref}}^{2}=17 \mathrm{GeV}^{2}\right)$, according to the fit ansatz in Eq. (17).

\begin{tabular}{lcccccc}
\hline \hline$\beta$ & Ansatz & $z_{-1}$ & $z_{0}$ & $z_{1}$ & $z_{2}$ & $\chi^{2} /$ d.o.f. \\
\hline 1.726 & 1 & & $0.4762(28)$ & $0.00190(23)$ & $-0.00021(59)$ & 0.4 \\
& 2 & $-0.011(43)$ & $0.4782(52)$ & $0.00178(13)$ & & 0.4 \\
1.778 & 1 & & $0.4828(39)$ & $0.00117(25)$ & $0.00053(56)$ & 0.3 \\
& 2 & $0.033(42)$ & $0.4772(40)$ & $0.00147(10)$ & & 0.3 \\
1.836 & 1 & & $0.4888(29)$ & $0.00101(19)$ & $-0.00032(43)$ & 0.03 \\
& 2 & $-0.021(30)$ & $0.4922(34)$ & $0.00083(08)$ & & 0.04 \\
\hline \hline
\end{tabular}

A dependence on $p^{2}$ of the $Z_{P}\left(\mu_{\text {ref }}^{2}\right)$ estimators in Fig. 3 is expected due to lattice artifacts, i.e., $\mathcal{O}\left(a^{2} p^{2}\right)$ terms, and possibly also to residual $\mathcal{O}\left(a^{0}\right)$ hadronic contaminations, ${ }^{2}$ which, however, must vanish as $1 / p^{2}$ at large $p^{2}$. The plots of $Z_{P}\left(\mu_{\mathrm{ref}}^{2}\right)$ in Fig. 3 show very good linearity in $p^{2}$ within the range $p^{2} \in[15,24] \mathrm{GeV}^{2}$, which is the one relevant for our determination of $Z_{P}$ and hence of the renormalized quark masses. This fact indicates that lattice artifacts other than $a^{2} p^{2}$-terms and possible hadronic contamination effects are negligible within our small statistical errors. Such a property is explicitly checked at each $\beta$ value by performing a fit of $Z_{P}\left(\mu_{\text {ref }}^{2}=17 \mathrm{GeV}^{2}\right)$ (left panel of Fig. 3) with the two Ansätze given by

Ansatz 1: $Z_{P}\left(\mu_{\text {ref }}^{2}\right)=z_{0}+z_{1} p^{2}+z_{2}\left(p^{2}\right)^{2}$,

Ansatz 2: $Z_{P}\left(\mu_{\mathrm{ref}}^{2}\right)=z_{0}+z_{1} p^{2}+\frac{z_{-1}}{p^{2}}$.

The resulting best fit values for $z_{i}$ are given in Table IV. The coefficients $z_{2}$ and $z_{-1}$ are compatible with zero within statistical errors at all $\beta$ values, while the coefficients $z_{1}$ scale nicely with $a^{2}$ (see Table II). From this check, we see that the systematic uncertainties on $Z_{P}$ are negligible within our small statistical errors. We will comment on the value of $z_{0}$ when we present our results in Sec. IV C.

Within the present study of renormalized quark masses, we follow two different methods for determining $Z_{P}$ in the RI' scheme and use data in two different $p^{2}$-ranges. The first method (M1) consists in fitting the $Z_{P}\left(\mu_{\text {ref }}^{2}\right)$ data linearly in $p^{2}$ in a given $p^{2}$-range with the aim of removing $\mathcal{O}\left(a^{2} p^{2}\right)$ discretization effects, while in the second method (M2), the same data are fitted to a constant [12]. Method M2 is by construction much less sensitive than M1 to possible small residual hadronic contaminations but at the expense of leaving some $\mathcal{O}\left(a^{2}\right)$ artifacts in the determination of $Z_{P}$. The ranges of $p^{2}$ used in the present analysis are $p^{2} \in$ $[15,19] \mathrm{GeV}^{2}$ and $p^{2} \in[18,24] \mathrm{GeV}^{2}$ for determining $Z_{P}\left(17 \mathrm{GeV}^{2}\right)$ and $Z_{P}\left(21 \mathrm{GeV}^{2}\right)$, respectively.

As an additional check of our determination of $Z_{P}\left(\mu^{2}\right)$ in the $\mathrm{RI}^{\prime}-\mathrm{MOM}$ scheme, we show in Fig. 4 the results for the nonperturbative step scaling function

\footnotetext{
${ }^{2}$ This point is discussed, e.g., in the Appendix of Ref. [23].
}

$\Sigma_{P}\left(\mu_{A}^{2}, \mu_{B}^{2}\right)=Z_{P}\left(\mu_{A}^{2}\right) / Z_{P}\left(\mu_{B}^{2}\right) \quad$ versus $\left(a / w_{0}\right)^{2}$ for $\left(\mu_{A}^{2}, \mu_{B}^{2}\right)=(21.5,14.3) \mathrm{GeV}^{2}$. We see that the lattice QCD data exhibit small discretization errors and agree in the continuum limit with the perturbative counterpart $\Sigma_{P}^{\mathrm{pt}}\left(\mu_{A}^{2}, \mu_{B}^{2}\right)=1.058$, which is computed to $\mathrm{N}^{3} \mathrm{LO}$ [26].

Moreover, as will be shown in Sec. $\mathrm{V}$, using the four $Z_{P}$ determinations corresponding to the methods M1 and M2 and at the two reference scales $\mu_{\text {ref }}^{2}=17 \mathrm{GeV}^{2}$ and $\mu_{\text {ref }}=21 \mathrm{GeV}^{2}$, we obtain in the continuum limit consistent final results for the renormalized quark masses.

\section{B. Chiral extrapolation and Goldstone boson pole subtraction}

A crucial step in determining the renormalization constant $Z_{P}$ is the extrapolation of its lattice estimators to the chiral limit, where the mass-independent $\mathrm{RI}^{\prime}-\mathrm{MOM}$ scheme is defined.

\section{Hadronic contaminations in the pseudoscalar vertex}

It is well known that the pseudoscalar vertex $\mathcal{V}_{P}$ receives contributions at the nonperturbative level by hadronic contaminations whose leading term scales as approximately $\left(p^{2} m_{\pi}^{2}\right)^{-1}$ [23]. Such a Goldstone boson pole has to be identified and subtracted from the data. In a unitary

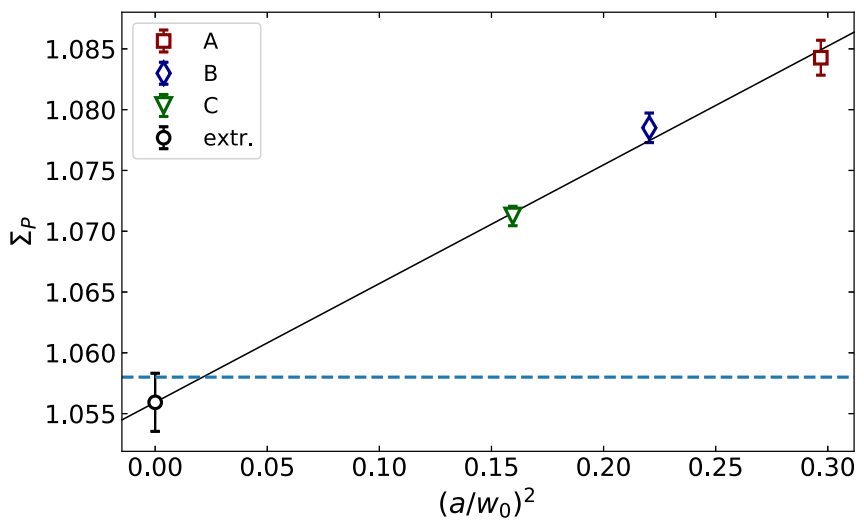

FIG. 4. The scaling function $\Sigma_{P}\left(\mu_{A}^{2}, \mu_{B}^{2}\right)=Z_{P}\left(\mu_{A}^{2}\right) / Z_{P}\left(\mu_{B}^{2}\right)$ vs $\left(a / w_{0}\right)^{2}$ for the three $\beta$-values studied (red square for $\beta=1.726$, blue rhombus for $\beta=1.778$, and green triangle for $\beta=1.836$ ) as well as the continuum extrapolation (black circle). The dashed line shows the perturbative value, $\Sigma_{P}^{\mathrm{pt}}\left(\mu_{A}^{2}, \mu_{B}^{2}\right)=1.058$. 
lattice setup for QCD with $N_{f}=4$ degenerate flavors of mass $m_{q}$, the lattice estimator of the vertex, $v_{P}\left(p^{2}, m_{q}\right)$, is expected to have the form

$$
\begin{aligned}
v_{P}\left(p^{2}, m_{q}\right)= & \mathcal{V}_{P}\left(p^{2}\right)+h \frac{\Lambda_{\mathrm{QCD}}^{4}}{p^{2} m_{\pi}^{2}}+h^{\prime} \frac{\Lambda_{\mathrm{QCD}}^{2}}{p^{2}} \\
& +h^{\prime \prime} m_{q} \frac{\Lambda_{\mathrm{QCD}}}{p^{2}}+\cdots,
\end{aligned}
$$

where the dimensionless quantities $\mathcal{V}_{P}$ (our target vertex), $h, h^{\prime}$, and $h^{\prime \prime}$ depend in general on $a^{2} p^{2}, a^{2} \Lambda_{\mathrm{QCD}}^{2}$, $a^{2} m_{q} \Lambda_{\mathrm{QCD}}$, and $a^{2} m_{q}^{2}$, while the ellipses stand for terms suppressed by higher powers of $1 / p^{2}$ as $p^{2} \rightarrow \infty$. We note that terms linear in $m_{q}$ are either hadronic contaminations suppressed as approximately $1 / p^{2}$ at large $p^{2}$ or lattice artifacts of the form approximately $a^{2} m_{q} \Lambda_{\mathrm{QCD}}$, which are numerically tiny for the $a m_{q}$ values of interest here. Since close to the chiral limit $m_{\pi}^{2} \sim m_{q}$, an equivalent Ansatz for $v_{P}\left(p^{2}, m_{q}\right)$ can be written in the form ${ }^{3}$

$v_{P}\left(p^{2}, m_{q}\right)=\mathcal{V}_{P}\left(p^{2}\right)+\frac{1}{p^{2}}\left(\kappa m_{q}^{-1}+\kappa^{\prime}+\kappa^{\prime \prime} m_{q}\right)+\ldots$,

where we separate the hadronic contaminations decreasing, for large $p^{2}$, like $1 / p^{2}$ from the vertex of interest $\mathcal{V}_{P}\left(p^{2}\right)$.

\section{Choice of a partially quenched setup}

In a PQ setup, such as the one adopted in the present analysis (see Sec. IV A), the lattice action is power counting renormalizable, and the operator vertices evaluated at several values of valence $\left(\mu_{\text {val }}\right)$ and sea $\left(\mu_{\text {sea }}\right)$ quark masses approach, as $\left(\mu_{\text {val }}, \mu_{\text {sea }}\right) \rightarrow(0,0)$, the corresponding unitary vertices from which the $\mathrm{RI}^{\prime}-\mathrm{MOM}$ renormalization constants can be computed. As detailed in Sec. IV A above, at all $\beta$ values, we use nine values of $\mu_{\text {val }}$ for each of the four $\mu_{\text {sea }}$ values. This allows us to have a good control on the mass dependence of the pseudoscalar vertex and to adopt, at fixed $\beta, p^{2}$, and $\mu_{\text {sea }}$ values, the following fit Ansatz for the chiral fit in $\mu_{\mathrm{val}}$,

$$
\begin{aligned}
v_{P}\left(p^{2}, \mu_{\mathrm{val}}, \mu_{\mathrm{sea}}\right)= & \mathcal{V}_{P}\left(p^{2}, \mu_{\mathrm{sea}}\right)+H \frac{\Lambda_{\mathrm{QCD}}^{4}}{p^{2}\left[m_{\pi}^{2}\right]_{\mathrm{val}}} \\
& +H^{\prime} \frac{\Lambda_{\mathrm{QCD}}^{2}}{p^{2}}+H^{\prime \prime} \mu_{\mathrm{val}} \frac{\Lambda_{\mathrm{QCD}}}{p^{2}}+\cdots,
\end{aligned}
$$

where the quantities $\mathcal{V}_{P}\left(p^{2}, \mu_{\text {sea }}\right), H, H^{\prime}$, and $H^{\prime \prime}$ depend, besides on $\mu_{\text {sea }}$ (to a numerically negligible level, as we shall see below), also on $a^{2} p^{2}, a^{2} \Lambda_{\mathrm{QCD}}^{2}, a^{2} m_{q} \Lambda_{\mathrm{QCD}}$, and $a^{2} m_{q}^{2}$, while the ellipses have the same meaning as in Eq. (18). Noting that the hadronic contaminations in the

\footnotetext{
${ }^{3}$ Due to next-to-leading-order terms in the chiral expansion of $m_{\pi}^{2}$, here $\kappa^{\prime}$ does not coincide with $h^{\prime} \Lambda_{\mathrm{QCD}}^{2} / p^{2}$.
}

three-point correlation function of quark, pseudoscalar bilinear, and antiquark fields at fixed 4-momenta (and in the derived quantity $v_{P}$ ) arise from the time orderings where the quark and antiquark fields are located at time distances both before or both after the pseudoscalar density [23], it follows that the Goldstone boson pole contamination is controlled by the mass $\left[m_{\pi}^{2}\right]_{\mathrm{val}}$ of the valence pion that appears as an intermediate state in the aforementioned time orderings. Recalling also that, to leading order in PQ chiral perturbation theory, $\left[m_{\pi}^{2}\right]_{\mathrm{val}} \sim \mu_{\mathrm{val}}$ [27], we choose to use the equivalent Ansatz

$$
\begin{aligned}
& v_{P}\left(p^{2}, \mu_{\text {val }}, \mu_{\text {sea }}\right) \\
& \quad=\mathcal{V}_{P}\left(p^{2}, \mu_{\text {sea }}\right)+\frac{K}{p^{2}} \frac{1}{\mu_{\text {val }}}+\frac{K^{\prime}}{p^{2}}+\frac{K^{\prime \prime}}{p^{2}} \mu_{\text {val }}+\cdots,
\end{aligned}
$$

where again we separate the hadronic contaminations (suppressed like $1 / p^{2}$ as $p^{2} \rightarrow \infty$ ) from the vertex of interest and the dimensionful coefficients $K, K^{\prime}$, and $K^{\prime \prime}$ in general depend on $\mu_{\text {sea }}$ and may be affected by lattice artifacts. From the fit of $v_{P}$ in $\mu_{\text {val }}$ at fixed $p^{2}$ and $\mu_{\text {sea }}$, one can determine the coefficients $K$ and $K^{\prime \prime}$, but it is not possible to disentangle the vertex $\mathcal{V}_{P}\left(p^{2}, \mu_{\text {sea }}\right)$ from the hadronic contamination $K^{\prime} / p^{2}$. At this point, one can safely take the limit $\mu_{\text {sea }} \rightarrow 0$ and determine $\mathcal{V}_{P}\left(p^{2}, 0\right)+\left.K^{\prime}\right|_{\mu_{\text {sea }}=0} / p^{2}$. After taking the full chiral limit, one can check that the residual hadronic contamination $\left.K^{\prime}\right|_{\mu_{\text {sea }}=0} / p^{2}$ is completely negligible in the range of $p^{2}$ used for the extraction of $Z_{P}$, as already detailed in Sec. IVA (see the discussion around Table IV).

\section{Numerical data and intermediate analysis results}

Within the $p^{2}$ ranges used in the present analysis, our data for $v_{P}\left(p^{2}, \mu_{\text {val }}, \mu_{\text {sea }}\right)$ exhibit a tiny linear dependence on $\mu_{\text {val }}$, which is compatible with $K^{\prime \prime}=\mathcal{O}\left(a^{2}\right)$ up to statistical errors. This is checked by fitting the data to the Ansatz of Eq. (21) for each fixed $a \mu_{\text {sea }}$ and $p^{2}$, which

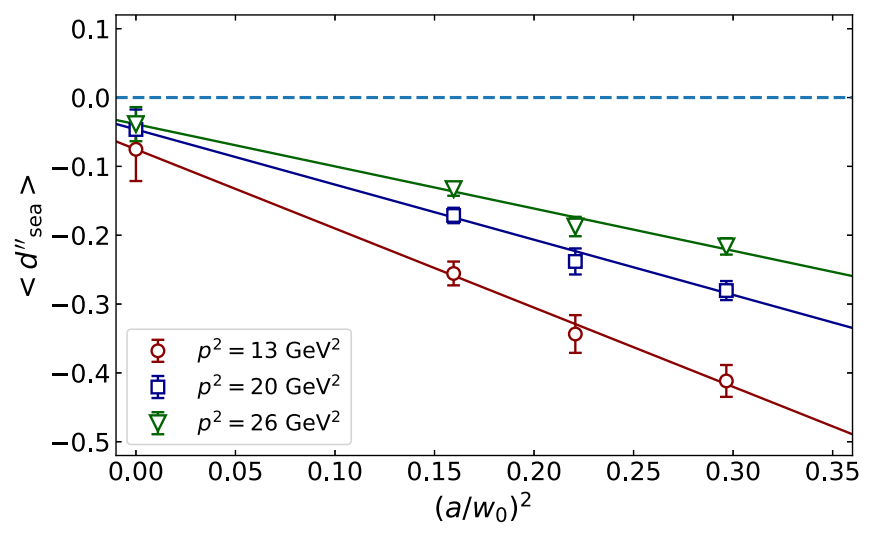

FIG. 5. Scaling of the coefficient $d^{\prime \prime} \equiv K^{\prime \prime} /\left(w_{0} p^{2}\right)$ averaged over $\mu_{\text {sea }},\left\langle d^{\prime \prime}\right\rangle_{\text {sea }}$. The three curves correspond to different values of $p^{2}$, namely, 13,20 , and $26 \mathrm{GeV}^{2}$. 

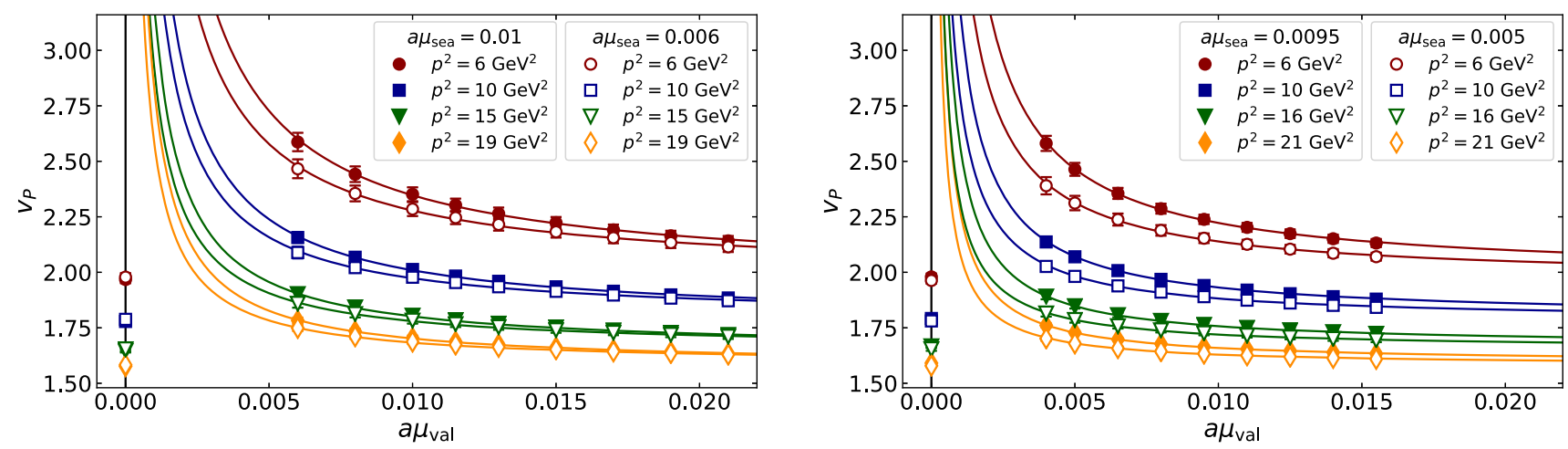

FIG. 6. Fit of the pseudoscalar vertex lattice estimators $v_{P}\left(p^{2}, \mu_{\mathrm{val}}, \mu_{\mathrm{sea}} ; a\right)$ according to the Ansatz of Eq. (22) for $\beta=1.726$ (left panel) and $\beta=1.836$ (right panel). Different colors correspond to different values of $p^{2}$, while full and empty circles correspond to different values of the sea quark mass $a \mu_{\text {sea }}$. The extrapolated values at $a \mu_{\text {val }}=0$ correspond to the quantities $\left[\mathcal{V}_{P}\left(p^{2}, \mu_{\text {sea }}\right)+K^{\prime} / p^{2}\right]$ in Eq. (22).

determines the quantities $\left[\mathcal{V}_{P}\left(p^{2}, \mu_{\text {sea }}\right)+K^{\prime} / p^{2}\right], K / p^{2}$, and $K^{\prime \prime} / p^{2}$, and then studying the dimensionless ratio $d^{\prime \prime} \equiv$ $K^{\prime \prime} /\left(w_{0} p^{2}\right)$ as a function of $\left(a / w_{0}\right)^{2}$. At fixed $\beta$ value, we observe that $d^{\prime \prime}$ changes with the sea quark mass of a given gauge ensemble nonmonotonically in $\mu_{\text {sea }}$ by the same amount as the statistical errors. Therefore, we average over the values of $d^{\prime \prime}$ at each $\mu_{\text {sea }}$ for a fixed $\beta$ value, obtaining the quantity $\left\langle d^{\prime \prime}\right\rangle_{\text {sea }}$, which is shown in Fig. 5 for three representative $p^{2}$ values, namely, 13,20 , and $26 \mathrm{GeV}^{2}$. As one can see, the continuum limit of $d^{\prime \prime}$ is consistent with zero up to statistical errors and/or a small residual term, which, even if present (given the numerical values of $\left.a \mu_{\text {val }} \lesssim 0.02\right)$, would alter $Z_{P}$ only to $\mathcal{O}\left(10^{-4}\right)$. In view of the evidence that $K^{\prime \prime}$ is $\mathcal{O}\left(a^{2}\right)$ or numerically negligible in the $p^{2}$ range of interest here, we can perform the fit in $\mu_{\mathrm{val}}$ on the data for $v_{P}$ excluding the term linear in $\mu_{\mathrm{val}}$; namely, we use

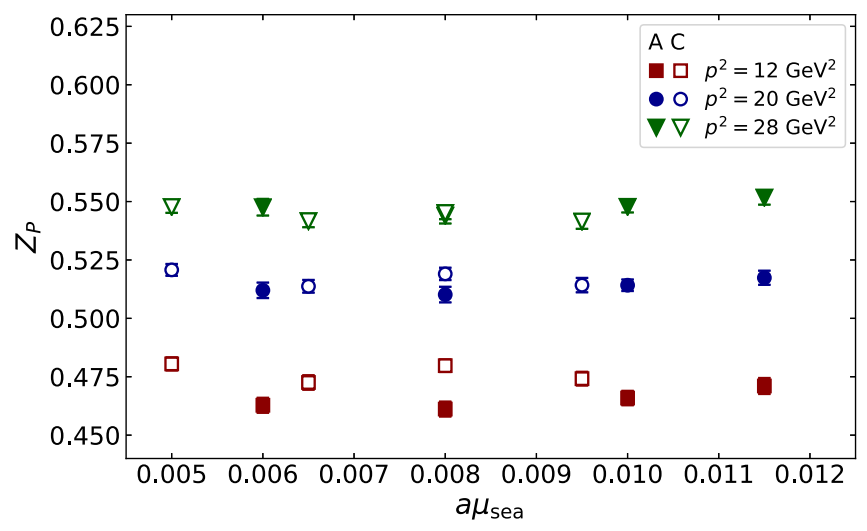

FIG. 7. Dependence of the renormalization constant $Z_{P}\left(\mu_{\text {sea }}, p^{2}\right)$ on the quark sea mass $\mu_{\text {sea }}$ at $\beta=1.726$ (A) and $\beta=1.836$ (C) for different values of the momentum $p^{2}=(12,20,28) \mathrm{GeV}^{2}$.
$v_{P}\left(p^{2}, \mu_{\mathrm{val}}, \mu_{\mathrm{sea}}\right)=\left[\mathcal{V}_{P}\left(p^{2}, \mu_{\mathrm{sea}}\right)+\frac{K^{\prime}}{p^{2}}\right]+\frac{K}{p^{2}} \frac{1}{\mu_{\mathrm{val}}}$.

This procedure has the advantage of yielding small statistical errors at the price of including well-controlled $\mathcal{O}\left(a^{2}\right)$ artifacts in the numerical estimate of $\left[\mathcal{V}_{P}\left(p^{2}, \mu_{\text {sea }}\right)+\right.$ $\left.K^{\prime} / p^{2}\right]$ and hence of $Z_{P}$.

The results of the fit on $v_{P}$ for few values of $p^{2}$ and the two extreme values of $\mu_{\text {sea }}$ are shown in Fig. 6 for the cases $\beta=1.726$ and $\beta=1.836$. Besides the very good quality of the fits, we remark that the resulting estimates of $\left[\mathcal{V}_{P}\left(p^{2}, \mu_{\text {sea }}\right)+K^{\prime} / p^{2}\right]$ at $\mu_{\text {val }}=0$ indeed show a very tiny dependence on $\mu_{\text {sea }}$ as mentioned above. Such a dependence on $\mu_{\text {sea }}$ turns out to be of the same size as the statistical errors (about $0.5 \%$ ), nonmonotonic in $\mu_{\text {sea }}$ at fixed $\beta$ and with different trends at different $\beta$ 's.

This feature is illustrated in Fig. 7, where the resulting estimates of $Z_{P}\left(\mu_{\text {sea }}, p^{2}\right)$, obtained using the RI condition of Eq. (8), are shown at $\beta=1.726$ and $\beta=1.836$. Therefore, as the dependence on $\mu_{\text {sea }}$ of $Z_{P}\left(\mu_{\text {sea }}, p^{2}\right)$ is not statistically significant, we average them in order to estimate $Z_{P}\left(p^{2}\right)$ in the unitary chiral limit.

TABLE V. Results for $Z_{P}$ in $\mathrm{RI}^{\prime}$-MOM for each $\beta$ value for methods M1 (second and fourth columns) and M2 (third and fifth columns), computed at reference scales of $\mu_{\text {ref }}=17 \mathrm{GeV}^{2}$ (indicated by "a") and $\mu_{\text {ref }}=21 \mathrm{GeV}^{2}$ (indicated by "b"). For cases M1a and M2a, results are extracted in the range $p^{2} \in(15,19) \mathrm{GeV}^{2}$, while for $\mathrm{M} 1 \mathrm{~b}$ and M2b, $p^{2} \in(18,24) \mathrm{GeV}^{2}$.

\begin{tabular}{lcccc}
\hline \hline \multicolumn{5}{c}{$\mathrm{RI}^{\prime}-\mathrm{MOM}$} \\
\hline$\beta$ & M1a & M2a & M1b & M2b \\
\hline 1.726 & $0.4774(24)$ & $0.5079(24)$ & $0.4917(26)$ & $0.5301(24)$ \\
1.778 & $0.4812(32)$ & $0.5042(26)$ & $0.4944(27)$ & $0.5255(23)$ \\
1.836 & $0.4899(26)$ & $0.5053(23)$ & $0.5046(27)$ & $0.5240(24)$ \\
\hline \hline
\end{tabular}


TABLE VI. Results for $Z_{P}$ in the RI'-MOM scheme but evolved to the common reference scale $\mu_{\text {ref }}^{2}=19 \mathrm{GeV}^{2}$. The notation is the same as that in Table V, and we report separately the statistical error and the systematic uncertainty related to the evolution factors.

\begin{tabular}{lcccr}
\hline \hline \multicolumn{5}{c}{$\mathrm{RI}^{\prime}-\mathrm{MOM}, \mu_{\mathrm{ref}}^{2}=19 \mathrm{GeV}^{2}$} \\
$\beta$
\end{tabular}

\section{Results for $Z_{P}$ in the $R I^{\prime}$-MOM and $\overline{M S}$ schemes}

In Table $\mathrm{V}$, we give the results of $Z_{P}$ determined in the RI'-MOM scheme using methods M1 and M2 (see Sec. IV A) for the two reference scales $\mu_{\text {ref }}^{2}=17 \mathrm{GeV}^{2}$ (labeled by "a") and $\mu_{\text {ref }}^{2}=21 \mathrm{GeV}^{2}$ (labeled by "b"). We find that at each $\beta$ value the parameter $z_{0}$ appearing in Eq. (17) is found to be compatible with the results for $Z_{P}\left(\mu_{\text {ref }}^{2}=17 \mathrm{GeV}^{2}\right)$ extracted using the method M1, as expected since this method corresponds to a linear fit Ansatz in $p^{2}$. The results are obtained by fitting the data in the momentum ranges $p^{2} \in$ $(15,19) \mathrm{GeV}^{2}$ and $p^{2} \in(18,24) \mathrm{GeV}^{2}$, respectively, for the reference scales 17 and $21 \mathrm{GeV}^{2}$. The values of $Z_{P}$ given in Table V (in the RI'-MOM scheme) are then evolved to the common reference scale $\mu_{\text {ref }}^{2}=19 \mathrm{GeV}^{2}$ and are reported in Table VI. The four determinations of $Z_{P}\left(\mu_{\mathrm{ref}}^{2}\right)(\mathrm{M} 1 \mathrm{a}, \mathrm{M} 2 \mathrm{a}$, $\mathrm{M} 1 \mathrm{~b}$, and M2b) are equally good estimates of the renormalization constant that only differ by $\mathcal{O}\left(a^{2}\right)$ cutoff effects. This implies that using whichever of them leads to equivalent results for the renormalized quark masses and renormalized matrix elements of the pseudoscalar density in the continuum limit. As a check of the good accuracy to which this property

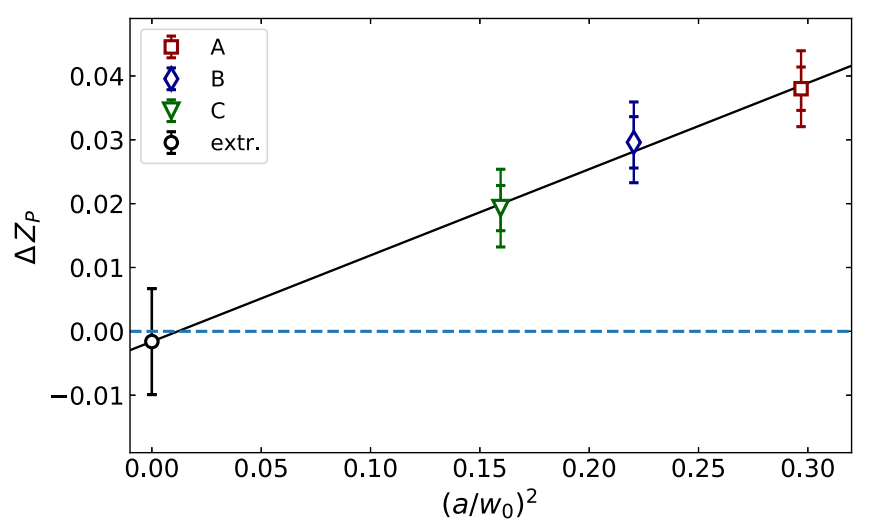

FIG. 8. $\Delta Z_{P}=Z_{P}[\mathrm{M} 2 \mathrm{~b}]-Z_{P}[\mathrm{M} 1 \mathrm{a}]$ vs $\left(a / w_{0}\right)^{2}$ and its (linear) continuum extrapolation. At each finite value of $a$, the smaller error bars correspond to the first (statistical) error in Table VI, while the larger ones also include the second error in the same Table, which is due to the $\mathrm{N}^{3} \mathrm{LO}$ evolution to $19 \mathrm{GeV}^{2}$ and is independent from the lattice spacing. Therefore, the continuum limit value and its uncertainty are obtained by taking into account only the statistical errors at finite $a$. is expected to be satisfied, we show in Fig. 8 the scaling behavior of the difference $\Delta Z_{P}=Z_{P}[\mathrm{M} 2 \mathrm{~b}]-Z_{P}[\mathrm{M} 1 \mathrm{a}]$ (in the $\mathrm{RI}^{\prime}-\mathrm{MOM}$ scheme at $19 \mathrm{GeV}^{2}$ ), for which all logarithmic divergences cancel and the continuum limit vanishes. A similar behavior, but with smaller $\mathcal{O}\left(a^{2}\right)$ artifacts, is observed for all the analogous differences of the four $Z_{P}$ determinations in Table VI. Finally, in Table VII, we show the values of $Z_{P}\left(19 \mathrm{GeV}^{2}\right)$ converted to the $\overline{\mathrm{MS}}$ scheme.

Since quark masses are generally given in the $\overline{\mathrm{MS}}$ scheme at 2 or $3 \mathrm{GeV}$, we obtain the corresponding renormalization constants at these scales by using the following evolution factors:

$$
\begin{aligned}
& Z_{P}^{\overline{\mathrm{MS}}}\left(4 \mathrm{GeV}^{2}\right)=0.83416(86) Z_{P}^{\overline{\mathrm{MS}}}\left(19 \mathrm{GeV}^{2}\right), \\
& Z_{P}^{\overline{\mathrm{MS}}}\left(9 \mathrm{GeV}^{2}\right)=0.92570(34) Z_{P}^{\overline{\mathrm{MS}}}\left(19 \mathrm{GeV}^{2}\right), \\
& Z_{P}^{\overline{\mathrm{MS}}}\left(16 \mathrm{GeV}^{2}\right)=0.98359(19) Z_{P}^{\overline{\mathrm{MS}}}\left(19 \mathrm{GeV}^{2}\right)
\end{aligned}
$$

Our evolution function is accurate at $\mathrm{N}^{3} \mathrm{LO}$ [26], i.e., $\mathcal{O}\left(\alpha_{\mathrm{s}}^{3}\right)$, and therefore we estimate the uncertainty due to higher orders as the last known term raised to the power $4 / 3$ (see the second error in the results of Table VI). When computing conversion factors, which are ratios of evolution functions, we add in quadrature the error coming from the numerator and the denominator. We verified that this procedure provides a good estimate of the uncertainty due to higher orders when applied to the $\mathrm{N}^{2} \mathrm{LO}$ conversion factors in order to estimate the (known) $\mathrm{N}^{3} \mathrm{LO}$ results.

TABLE VII. Results for $Z_{P}$ at the scale $\mu_{\text {ref }}^{2}=19 \mathrm{GeV}^{2}$ converted from $\mathrm{RI}^{\prime}$-MOM (results of Table VI) to the $\overline{\mathrm{MS}}$ scheme.

\begin{tabular}{lcccc}
\hline \hline \multicolumn{5}{c}{$\overline{\mathrm{MS}}, \mu_{\text {ref }}^{2}=19 \mathrm{GeV}^{2}$} \\
\hline$\beta$ & M1a & M2a & M1b & M2b \\
\hline 1.726 & $0.569(3)(5)$ & $0.605(3)(5)$ & $0.569(3)(5)$ & $0.614(3)(5)$ \\
1.778 & $0.574(4)(5)$ & $0.601(3)(5)$ & $0.572(3)(5)$ & $0.608(3)(5)$ \\
1.836 & $0.584(3)(5)$ & $0.602(3)(5)$ & $0.584(3)(5)$ & $0.607(3)(5)$ \\
\hline \hline
\end{tabular}


TABLE VIII. Values of the bare valence quark mass parameters and the corresponding values of $m_{\pi}$ and $f_{\pi}$ for each of the ensembles used in the analysis in the pion sector. In the last column the number of gauge configurations analyzed for each ensemble is presented.

\begin{tabular}{llllrr}
\hline \hline Ensemble & $a \mu_{\ell}$ & $a \mu_{\ell}$ & $a m_{\pi}$ & \multicolumn{1}{c}{$a f_{\pi}$} & Confs \\
\hline cA211.53.24 & 0.0053 & 0.0053 & $0.16626(51)$ & $0.07106(36)$ & 628 \\
cA211.40.24 & 0.0040 & 0.0040 & $0.14477(70)$ & $0.06809(30)$ & 662 \\
cA211.30.32 & 0.0030 & 0.0030 & $0.12530(16)$ & $0.06674(15)$ & 1237 \\
cA211.12.48 & 0.0012 & 0.0012 & $0.08022(18)$ & $0.06133(33)$ & 322 \\
cB211.25.32 & 0.0025 & 0.0025 & $0.10475(45)$ & $0.05652(38)$ & 400 \\
cB211.25.48 & 0.0025 & 0.0025 & $0.10465(14)$ & $0.05726(12)$ & 314 \\
cB211.14.64 & 0.0014 & 0.0014 & $0.07848(10)$ & $0.05477(12)$ & 437 \\
cB211.072.64 & 0.00072 & 0.00072 & $0.05659(8)$ & $0.05267(14)$ & 374 \\
cC211.20.48 & 0.0020 & 0.0020 & $0.08540(17)$ & $0.04892(13)$ & 890 \\
cC211.06.80 & 0.0006 & 0.0006 & $0.04720(7)$ & $0.04504(10)$ & 401 \\
\hline \hline
\end{tabular}

\section{MESON SECTOR ANALYSIS}

In this section, we describe the determination of the quark masses taking as input the isosymmetric values of the pion, kaon, and $D_{(s)}$-meson masses.

\section{A. Methodology}

For each ensemble, we compute the two-point function

$$
C(t)=\frac{1}{L^{3}} \sum_{\vec{x}, \vec{z}}\left\langle 0\left|J_{f f^{\prime}}(\vec{x}, t) J_{f f^{\prime}}^{\dagger}(\vec{z}, 0)\right| 0\right\rangle,
$$

where $J_{f f^{\prime}}(x)=\bar{q}_{f}(x) i \gamma_{5} q_{f^{\prime}}(x)$ is the meson interpolating field with $q_{f}$ being the valence quark field of flavor $f \in\{\ell, s, c\}$. By $\ell$, we denote the average up/down (light) quark. The correlators for the pion and kaon are the same as those used in Ref. [11]. For all mesons, the two valence quarks $q_{f}$ and $q_{f^{\prime}}$ are always taken with opposite Wilson parameters, i.e., $r_{f}=-r_{f^{\prime}}=1$, as this choice is known to suppress $\mathrm{O}\left(a^{2}\right)$ lattice artifacts $\left.[4,7]\right)$. For the valence mass parameters, we evaluate correlators at $\mu_{\ell}$ values equal to its sea counterpart, as well as at three values of the quark mass parameter $\mu_{s}$ in the range of the strange quark masses and four values of the quark mass parameter $\mu_{c}$ in the range of the charm quark masses. The chosen values of valence quark masses are collected in Table VIII and allow for a precise interpolation to the physical strange and charm quark masses as determined by the kaon and $D$-meson masses in the isosymmetric QCD. The latter ones, following the FLAG report [17], are given by

$$
\begin{aligned}
& m_{K}^{\text {isoQCD }}=494.2(3) \mathrm{MeV}, \\
& m_{D}^{\text {isoQCD }}=1867.0(4) \mathrm{MeV}, \\
& m_{D_{s}}^{\text {isoQCD }}=1969.0(4) \mathrm{MeV} .
\end{aligned}
$$

From the correlator given in Eq. (26), the overlap $\mathcal{S}=$ $\left|\left\langle\mathrm{PS}\left|J_{f f^{\prime}}\right| 0\right\rangle\right|^{2}$ can be extracted using an exponential fit at large time distances

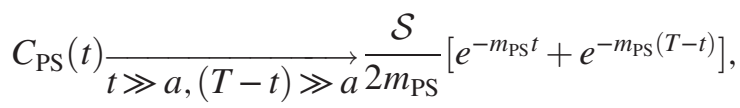

where $m_{\mathrm{PS}}$ is the ground-state mass of a pseudoscalar (PS) meson made of the two valence quarks with flavor $f$ and $f^{\prime}$. For maximally twisted quarks, the value of the matrix element $\mathcal{S}$ determines the PS-meson decay constant with no need of any renormalization constant [1], from the formula

$$
a f_{\mathrm{PS}}=a\left(\mu_{f}+\mu_{f^{\prime}}\right) \frac{\sqrt{a^{4} \mathcal{S}}}{a m_{\mathrm{PS}} \sinh \left(a m_{\mathrm{PS}}\right)} .
$$

The slight deviation from maximal twist of the ensemble cA211.12.48 is corrected according to Appendix C of Ref. [11].

The global energy scale is set using the isosymmetric QCD inputs (4), and data at different lattice spacings are connected by exploiting the gradient-flow (GF) quantities $w_{0}$ [28], $\sqrt{t_{0}}$ [29], and $t_{0} / w_{0}$ measured in lattice units. Their values have been already determined quite precisely in Ref. [11], namely,

$$
\begin{gathered}
w_{0}=0.17383(63) \mathrm{fm}, \\
\sqrt{t_{0}}=0.14436(61) \mathrm{fm}, \\
t_{0} / w_{0}=0.11969(62) \mathrm{fm} .
\end{gathered}
$$

Nevertheless, in order to take properly into account all the correlations with the meson data, the GF scales are determined again in the present analysis (see the next subsection), obtaining results very compatible with Eqs. (32)-(34).

\footnotetext{
${ }^{4}$ The result (32) improves drastically our preliminary value of $w_{0}$ obtained in Ref. [30]. The improvement is mainly related to a better description of discretization effects on the decay constant $f_{\pi}$ [see Eq. (36)] and to the replacement of $f_{\pi}$ with the quantity $\left(f_{\pi} m_{\pi}^{4}\right)^{1 / 5}$, which has been found to be less affected by lattice artifacts [11].
} 


\section{B. Light quark mass}

The lattice QCD data on the pion mass and decay constant are computed in a unitary setup, i.e., with $\mu_{\text {sea }}=\mu_{\text {valence }}=\mu_{\ell}$; the values used in this section are reported in Table VIII.

The lattice QCD data on the pion mass and decay constant are analyzed relying on $\mathrm{SU}(2)$ chiral perturbation theory (ChPT) using the formulas

$$
\begin{aligned}
\left(m_{\pi} w_{0}\right)^{2}= & 2\left(B w_{0}\right)\left(m_{\ell} w_{0}\right) \\
& \times\left[1+\xi_{\ell} \log \xi_{\ell}+P_{1} \xi_{\ell}+P_{2} a^{2} / w_{0}^{2}\right] K_{M^{2}}^{\mathrm{FSE},} \\
\left(f_{\pi} w_{0}\right)= & \left(f w_{0}\right)\left[1-2 \xi_{\ell} \log \xi_{\ell}+P_{3} \xi_{\ell}+P_{4} a^{2} / w_{0}^{2}\right. \\
& \left.+a^{2} m_{\ell} P_{5}\right] K_{f}^{\mathrm{FSE}},
\end{aligned}
$$

where the variable $\xi_{\ell}=2 B m_{\ell} /\left(16 \pi^{2} f^{2}\right)$ is related to the quark renormalized mass $m_{\ell}=\mu_{\ell} / Z_{P}$. The parameters $P_{1}$ and $P_{3}$ are related to the low-energy constants $\bar{\ell}_{3}$ and $\bar{\ell}_{4}$ by

$$
\begin{aligned}
& P_{1}=-\bar{\ell}_{3}-2 \log \left(m_{\pi}^{\text {isoQCD }} /(4 \pi f)\right), \\
& P_{3}=2 \bar{\ell}_{4}+4 \log \left(m_{\pi}^{\text {isoQCD }} /(4 \pi f)\right) .
\end{aligned}
$$

The quantities $K_{M^{2}}^{\mathrm{FSE}}$ and $K_{f}^{\mathrm{FSE}}$ represent the finite size effects (FSEs) on the squared pion mass and the pion decay constant, respectively. In Ref. [11], it was shown that SU(2) ChPT at next-to-leading order (NLO) [31] adequately describes our lattice data, once discretization effects proportional both to $a^{2}$ and to $a^{2} m_{\ell}$ are included in $f_{\pi}$ [see Eq. (36)], while in $m_{\pi}$, the leading lattice artifact is already directly proportional to $a^{2} m_{\ell}$ [see Eq. (35)]. The fit parameters are $B w_{0}, \bar{\ell}_{3}, P_{2}, f w_{0}, \bar{\ell}_{4}, P_{4}$, and $P_{5}$. We repeat the fit procedure adopting the values of the renormalization constant $Z_{P}$ determined using the methods M1a, M1b, M2a, and M2b given in Table VII.

To estimate possible systematics due to the scale setting and the chiral extrapolation, we repeat the analysis using:

(i) the ratio $t_{0} / w_{0}$ to set the scale;

(ii) the GF scale $\sqrt{t_{0}}$ to set the scale;

(iii) only a combination of two lattice spacing, ${ }^{5}$ namely, $\beta=1.726$ and $\beta=1.778$,

$\beta=1.726$ and $\beta=1.836$,

$\beta=1.778$ and $\beta=1.836$;

(iv) only ensembles with pion mass less than $190 \mathrm{MeV}$ and excluding the term $P_{5}$ in Eq. (36) from the fit.

The results for the light quark mass $m_{u d}$ are reported in Table IX, where we also include the values of the leading low-energy constants $B, f$, and $\Sigma^{1 / 3}=\left(B f^{2} / 2\right)^{1 / 3}$ as well as the values of $\chi^{2} /$ d.o.f. The chiral and continuum extrapolations are illustrated in Fig. 9.

We need now to average the results coming from the different analyses collected in Table IX. To this end,

\footnotetext{
${ }^{5}$ When considering the two finest lattice spacings $\beta=1.778$ and $\beta=1.836$, we exclude the term $P_{5}$ from the fit of Eq. (36) because all the ensembles have $m_{\pi}<260 \mathrm{MeV}$.
}

TABLE IX. The values of the light quark mass, $m_{u d}, B, f$, and $\Sigma$ in the $\overline{\mathrm{MS}}$ scheme at $2 \mathrm{GeV}$ obtained using the different determinations of $Z_{P}$, labeled M1a, M1b, M2a, and M2b. Results using the GF scale $w_{0}$ and all the ensembles of Table VIII are given in the top most panel, using $t_{0} / w_{0}$ in the second panel,

\begin{tabular}{|c|c|c|c|c|c|}
\hline$Z_{P}$ & $m_{u d}(\mathrm{MeV})$ & $B(\mathrm{MeV})$ & $f(\mathrm{MeV})$ & $\Sigma^{1 / 3}$ & $\chi^{2} /$ d.o.f. \\
\hline M1a & $3.677(65)$ & $2523(42)$ & $124.02(48)$ & 268.7(1.4) & 1.97 \\
\hline M2a & $3.694(64)$ & 2512(40) & $124.04(50)$ & $268.3(1.4)$ & 1.56 \\
\hline M1b & $3.677(66)$ & 2522(43) & $124.05(48)$ & $268.7(1.5)$ & 2.53 \\
\hline $\mathrm{M} 2 \mathrm{~b}$ & $3.694(65)$ & $2512(41)$ & $124.02(51)$ & $268.3(1.4)$ & 1.15 \\
\hline \multicolumn{6}{|c|}{$t_{0} / w_{0}$} \\
\hline M1a & $3.722(74)$ & $2493(47)$ & $124.40(49)$ & $268.2(1.6)$ & 2.91 \\
\hline M2a & $3.766(73)$ & $2465(45)$ & $124.48(52)$ & $267.3(1.5)$ & 2.54 \\
\hline M1b & $3.724(75)$ & 2492(48) & $124.42(49)$ & $268.2(1.6)$ & 3.72 \\
\hline $\mathrm{M} 2 \mathrm{~b}$ & $3.771(74)$ & 2462(45) & $124.48(52)$ & $267.2(1.6)$ & 1.95 \\
\hline \multicolumn{6}{|c|}{$\sqrt{t_{0}}$} \\
\hline M1a & $3.696(69)$ & $2510(45)$ & $124.19(48)$ & $268.5(1.5)$ & 2.48 \\
\hline M2a & $3.726(68)$ & 2491(43) & $124.24(51)$ & $267.9(1.5)$ & 2.06 \\
\hline M1b & $3.697(70)$ & $2509(45)$ & $124.22(48)$ & $268.5(1.5)$ & 3.18 \\
\hline $\mathrm{M} 2 \mathrm{~b}$ & $3.729(69)$ & 2490(43) & $124.23(51)$ & $267.8(1.5)$ & 1.55 \\
\hline \multicolumn{6}{|c|}{$w_{0}, m_{\pi}<190 \mathrm{MeV}, P_{5}=0$} \\
\hline M1a & $3.629(81)$ & $2572(55)$ & $122.71(45)$ & $268.5(2.3)$ & 4.72 \\
\hline M2a & $3.656(81)$ & $2554(53)$ & $122.56(48)$ & $267.7(2.2)$ & 3.4 \\
\hline M1b & $3.623(82)$ & $2577(56)$ & $122.72(45)$ & $268.7(2.3)$ & 6.32 \\
\hline $\mathrm{M} 2 \mathrm{~b}$ & $3.663(82)$ & $2547(54)$ & $122.51(49)$ & $267.4(2.3)$ & 2.2 \\
\hline \multicolumn{6}{|c|}{$w_{0}, \beta=1.726$ and $\beta=1.778$} \\
\hline M1a & $3.83(15)$ & $2420(93)$ & $124.54(83)$ & $265.8(3.2)$ & 0.0953 \\
\hline M2a & $3.83(14)$ & $2425(84)$ & $124.57(88)$ & $266.0(2.7)$ & 0.0892 \\
\hline M1b & $3.86(14)$ & 2402(86) & $124.54(84)$ & $265.1(2.8)$ & 0.0936 \\
\hline $\mathrm{M} 2 \mathrm{~b}$ & $3.80(13)$ & $2446(81)$ & $124.57(88)$ & $266.7(2.6)$ & 0.0895 \\
\hline \multicolumn{6}{|c|}{$w_{0}, \beta=1.726$ and $\beta=1.836$} \\
\hline M1a & $3.660(69)$ & $2539(44)$ & $122.30(49)$ & $266.8(1.5)$ & 0.104 \\
\hline M2a & $3.682(67)$ & $2525(43)$ & $122.22(51)$ & $266.2(1.5)$ & 0.0861 \\
\hline M1b & $3.657(70)$ & 2541(46) & $122.30(49)$ & $266.9(1.6)$ & 0.105 \\
\hline $\mathrm{M} 2 \mathrm{~b}$ & $3.685(68)$ & $2523(43)$ & $122.21(52)$ & $266.1(1.5)$ & 0.0851 \\
\hline \multicolumn{6}{|c|}{$w_{0}, \beta=1.778$ and $\beta=1.836, P_{5}=0$} \\
\hline M1a & $3.55(11)$ & $2614(77)$ & $122.64(16)$ & $269.9(2.8)$ & 0.356 \\
\hline M2a & $3.587(97)$ & $2588(68)$ & $122.49(17)$ & $268.8(2.5)$ & 0.323 \\
\hline M1b & $3.53(10)$ & 2631(73) & $122.66(16)$ & $270.5(2.6)$ & 0.362 \\
\hline $\mathrm{M} 2 \mathrm{~b}$ & $3.612(98)$ & $2571(67)$ & $122.44(17)$ & $268.1(2.5)$ & 0.315 \\
\hline
\end{tabular}
using $\sqrt{t_{0}}$ in the third panel, using $w_{0}$ and limiting $m_{\pi}<$ $190 \mathrm{MeV}$ in the fourth panel, using $w_{0}$ and only the two coarser lattice spacings in the fifth panel, using $w_{0}$ and only the coarser and finest lattice spacings in the sixth panel, and using $w_{0}$ and only the two finest lattice spacings in the last panel.

we adopt a simple generalization of Eq. (28) of Ref. [12]. For a given observable $x$, we assume that its probability distribution $f(x)$ is given by

$$
f(x)=\sum_{i=1}^{N} w_{i} f_{i}(x),
$$



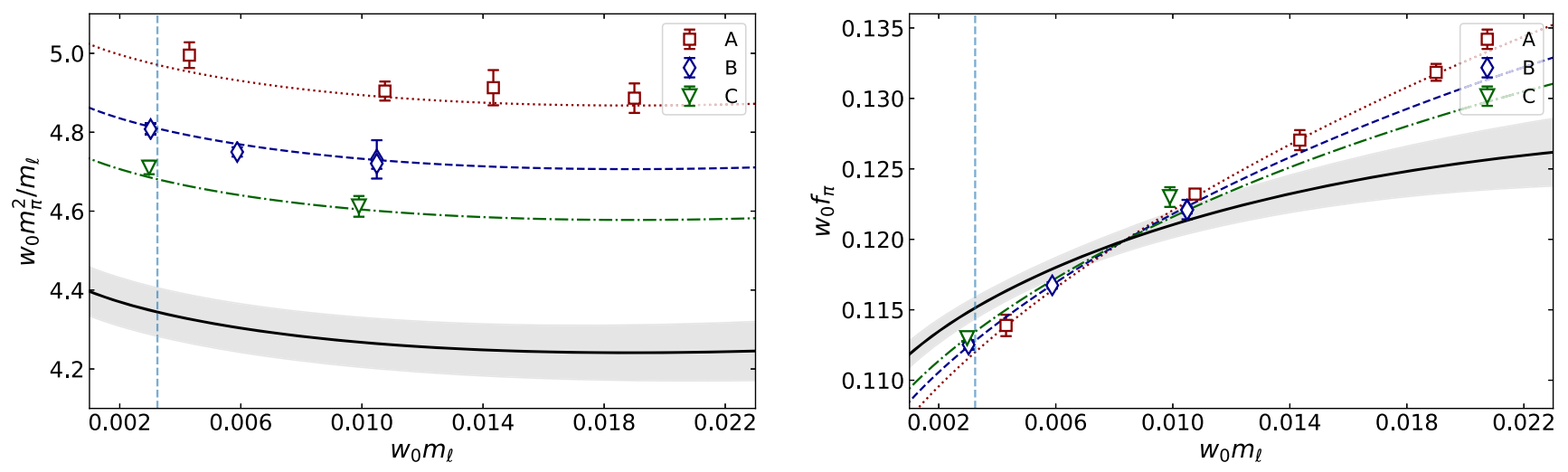

FIG. 9. Chiral and continuum extrapolation of $w_{0} m_{\pi}^{2} / m_{\ell}$ (left) and $w_{0} f_{\pi}$ (right) as function of $w_{0} m_{\ell}$ using Eqs. (35) and (36) and $Z_{P}$ for the M2b method. Different colored bands correspond to different lattice spacings (red for the A ensembles, blue for the B, and green for the C). The gray band is the extrapolation to the continuum limit. Note that for $w_{0} f_{\pi}$ discretization effects proportional both to $a^{2}$ and to $a^{2} m_{\ell}$ are visible (see the text).

where $w_{i}$ are weights to be specified and $f_{i}(x)$ are the probability distributions corresponding to the individual analyses (labeled with $i=1,2, \ldots, N$ ). It is not necessary to specify the form of the individual distributions. It suffices to know that $\bar{x}_{i}$ and $\sigma_{i}$ are the mean value and standard deviation of the distribution $f_{i}(x)$.

Thus, using Eq. (38), we can represent the combination of the $N$ results of the various analyses in the form

$$
\bar{x} \pm \sigma_{\text {stat }} \pm \sigma_{\text {syst }},
$$

where

$$
\begin{gathered}
\bar{x}=\sum_{i=1}^{N} w_{i} \bar{x}_{i}, \\
\sigma_{\text {stat }}^{2}=\sum_{i=1}^{N} w_{i} \sigma_{i}^{2}, \\
\sigma_{\text {syst }}^{2}=\sum_{i=1}^{N} w_{i}\left(\bar{x}_{i}-\bar{x}\right)^{2} .
\end{gathered}
$$

Equation (41) represents the square of a "statistical" error given by the weighted average of the individual variances, while Eq. (42) corresponds to the square of a "systematic" error related to the spread among the results of the different analyses. The total error $\sigma$ is given by the sum in quadrature of $\sigma_{\text {stat }}$ and $\sigma_{\text {syst }}$.

Given the limited number of data points, we refrain in using the values of $\chi^{2}$, shown in Table IX, as a quantitative estimate of the quality of the various fits. Instead, since the results of Table IX suggest the dominance of the statistical uncertainties over the systematic ones, a reasonable choice for the weights $w_{i}$ is $w_{i} \propto 1 / \sigma_{i}^{2}$, namely,

$$
w_{i}=\frac{1}{\sigma_{i}^{2}} \cdot \frac{1}{\sum_{j=1}^{N} 1 / \sigma_{j}^{2}} .
$$

Thus, to obtain the value of $m_{u d}$, we combine the values of Table IX using Eq. (40) for the mean and Eqs. (41)-(42) for the error excluding the analyses with $\chi^{2} /$ d.o.f. $>2.5$ (which leads to a total of 21 analyses). We get in this way

\begin{tabular}{|c|c|c|c|c|c|c|c|}
\hline \multirow{2}{*}{$\frac{\text { Ensemble }}{\mathrm{cA} 211.53 .24}$} & \multirow{2}{*}{$\frac{a \mu_{\ell}}{0.00530}$} & \multicolumn{3}{|c|}{$a \mu_{s}$} & \multicolumn{3}{|c|}{$a m_{K}$} \\
\hline & & 0.0176 & 0.022 & 0.0264 & $0.24134(47)$ & $0.26316(47)$ & $0.28340(47)$ \\
\hline cA211.40.24 & 0.00400 & 0.0176 & 0.022 & 0.0264 & $0.23419(51)$ & $0.25650(52)$ & $0.27709(52)$ \\
\hline cA211.30.32 & 0.00300 & 0.0176 & 0.022 & 0.0264 & $0.22810(21)$ & $0.25089(22)$ & $0.27185(22)$ \\
\hline cA211.12.48 & 0.00120 & 0.0176 & 0.022 & 0.0264 & $0.21789(26)$ & $0.24153(29)$ & $0.26319(34)$ \\
\hline cB211.25.32 & 0.00250 & 0.0148 & 0.0185 & 0.0222 & $0.19212(45)$ & $0.21143(46)$ & $0.22920(47)$ \\
\hline cB211.25.48 & 0.00250 & 0.0148 & 0.0185 & 0.0222 & $0.19141(19)$ & $0.21067(20)$ & $0.22838(23)$ \\
\hline cB211.14.64 & 0.00140 & 0.0148 & 0.0185 & 0.0222 & $0.18484(13)$ & $0.20460(15)$ & $0.22268(17)$ \\
\hline cB211.072.64 & 0.00072 & 0.0148 & 0.0185 & 0.0222 & $0.18038(14)$ & $0.20044(16)$ & $0.21875(18)$ \\
\hline cC211.20.48 & 0.00200 & 0.0128 & 0.0161 & 0.0193 & $0.16179(16)$ & $0.17878(17)$ & $0.19390(18)$ \\
\hline cC211.06.80 & 0.00060 & 0.0128 & 0.0161 & 0.0193 & $0.153321(99)$ & $0.17098(11)$ & $0.18656(13)$ \\
\hline
\end{tabular}

$m_{u d}=3.689(80)_{\text {stat }}(63)_{\text {syst }} \mathrm{MeV}=3.689(102) \mathrm{MeV}$,

TABLE X. Values of the bare valence quark mass parameters and the corresponding values of $a m_{K}$ for each of the ensembles used in the analysis in the kaon. The number of configuration analysed for each ensemble is the reported in Table VIII. 


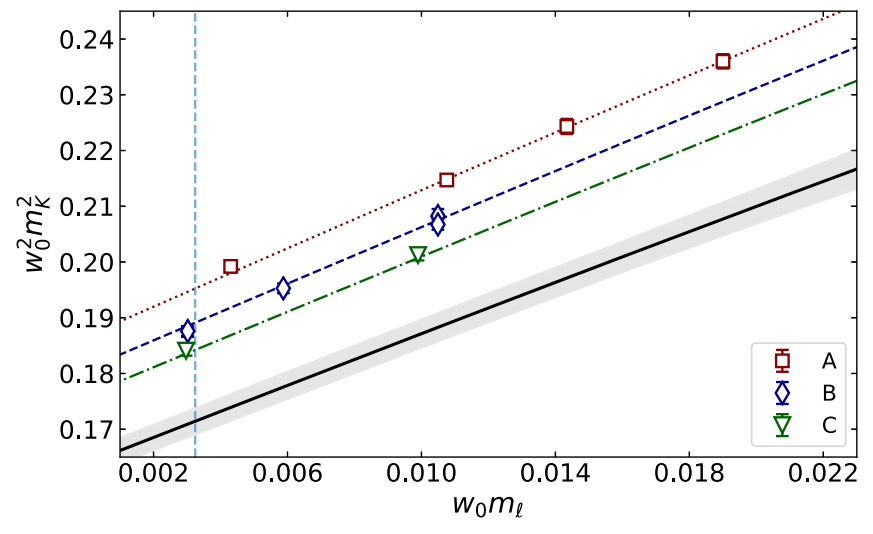

FIG. 10. The red, blue, and green solid lines show the resulting fits using Eq. (49) for ensembles A, B, and C respectively. The gray line shows the continuum extrapolation. We use $\left(m_{s} w_{0}\right)_{\text {ref }}=$ 0.080 and the $Z_{P}$ computed with method M2b.

$$
\begin{aligned}
& B=2516(51)_{\text {stat }}(42)_{\text {syst }} \mathrm{MeV}=2516(67) \mathrm{MeV} \\
& f=122.82(32)_{\text {stat }}(65)_{\text {syst }} \mathrm{MeV}=122.82(73) \mathrm{MeV}, \\
& \Sigma^{1 / 3}=267.6(1.8)_{\text {stat }}(1.1)_{\text {syst }} \mathrm{MeV}=267.6(2.1) \mathrm{MeV}
\end{aligned}
$$

\section{Strange quark mass}

In this section, we present our determination of the strange quark mass $m_{s}$. For the valence mass parameters, we evaluate correlators for $\mu_{\ell}$ values equal to its sea counterpart, as well as at three values of the quark mass parameter $\mu_{s}$ in the range of the strange quark masses shown in Table X.

For each ensemble, we perform a linear interpolation of the kaon mass to three reference values of $\left(m_{s} w_{0}\right)_{\text {ref }}=$ 0.064, 0.080, 0.095 using the Ansatz

$$
m_{K}^{2}=a+b m_{s} w_{0}
$$

A similar interpolation is also performed for the other GF scales $t_{0} / w_{0}$ and $\sqrt{t_{0}}$. Then, for each value of $\left(m_{s} w_{0}\right)_{\text {ref }}$, we extrapolate to the continuum limit and to the isosymmentric QCD point using the value of $m_{\ell}=m_{u d}$ determined in the previous section and our best fit to the data for $m_{K}$ according to the Ansatz

$$
\begin{aligned}
\left(m_{K} w_{0}\right)^{2}= & P_{0}\left(m_{\ell} w_{0}+m_{s} w_{0}\right) \\
& \times\left[1+P_{1} m_{\ell} w_{0}+P_{2} m_{\ell}^{2} w_{0}^{2}+P_{3} a^{2} / w_{0}^{2}\right] .
\end{aligned}
$$

At NLO order of SU(2) ChPT, there are no finite volume effects on the kaon mass, and in Ref. [11], it has been shown that the lattice QCD data on the kaon masses agree with this prediction. The fit parameters in Eqs. (49) are $P_{0}$, $P_{1}, P_{2}$, and $P_{3}$, while the LO low-energy constants $f$ and $B$ are taken from our pion sector fit. The quality of the resulting fit to Eq. (49) is shown in Fig. 10 as an example
TABLE XI. The values of the strange quark mass, $m_{s}$, in the $\overline{\mathrm{MS}}$ scheme at $2 \mathrm{GeV}$ and the ratio $m_{s} / m_{u d}$ obtained using the different determinations of $Z_{P}$, labeled M1a, M1b, M2a, and M2b. Results using the GF scale $w_{0}$ and all the ensembles of Table $\mathrm{X}$ are given in the top most panel, using $t_{0} / w_{0}$ in the second panel, using $\sqrt{t_{0}}$ in the third panel, using $w_{0}$ and limiting $m_{\pi}<$

\begin{tabular}{|c|c|c|c|}
\hline$Z_{P}$ & $m_{s}(\mathrm{MeV})$ & $m_{s} / m_{u d}$ & $\chi^{2} /$ d.o.f. \\
\hline M1a & $100.4(1.6)$ & $27.32(21)$ & 1 \\
\hline M2a & $100.9(1.5)$ & $27.32(22)$ & 1 \\
\hline M1b & $100.6(1.6)$ & $27.37(22)$ & 1.7 \\
\hline \multirow[t]{2}{*}{$\mathrm{M} 2 \mathrm{~b}$} & $101.0(1.5)$ & $27.34(22)$ & 0.76 \\
\hline & \multicolumn{3}{|c|}{$t_{0} / w_{0}$} \\
\hline M1a & $101.6(1.8)$ & $27.28(23)$ & 1.1 \\
\hline M2a & $102.8(1.7)$ & $27.30(24)$ & 1.3 \\
\hline M1b & $101.8(1.8)$ & $27.34(23)$ & 1.9 \\
\hline \multirow[t]{2}{*}{$\mathrm{M} 2 \mathrm{~b}$} & $103.1(1.7)$ & $27.34(24)$ & 1 \\
\hline & \multicolumn{3}{|c|}{$\sqrt{t_{0}}$} \\
\hline M1a & $101.0(1.7)$ & $27.31(22)$ & 1.1 \\
\hline M2a & $101.8(1.6)$ & $27.32(23)$ & 1.2 \\
\hline M1b & 101.2(1.7) & $27.37(22)$ & 1.8 \\
\hline \multirow[t]{2}{*}{$\mathrm{M} 2 \mathrm{~b}$} & $102.0(1.6)$ & $27.35(23)$ & 0.9 \\
\hline & \multicolumn{3}{|c|}{$w_{0}, m_{\pi}<190 \mathrm{MeV}, P_{2}=0$} \\
\hline M1a & $100.9(1.8)$ & $27.82(40)$ & 3.6 \\
\hline M2a & $101.4(1.7)$ & $27.73(37)$ & 4 \\
\hline M1b & 101.1(1.8) & $27.90(38)$ & 6.1 \\
\hline \multirow[t]{2}{*}{$\mathrm{M} 2 \mathrm{~b}$} & $101.4(1.7)$ & $27.68(37)$ & 2.9 \\
\hline & \multicolumn{3}{|c|}{$w_{0}, \beta=1.726$ and $\beta=1.778$ only, $P_{2}=0$} \\
\hline M1a & $103.4(3.7)$ & $26.96(29)$ & 0.34 \\
\hline M2a & $103.1(3.2)$ & $26.94(31)$ & 0.46 \\
\hline M1b & $104.1(3.4)$ & $26.95(29)$ & 0.42 \\
\hline \multirow[t]{2}{*}{$\mathrm{M} 2 \mathrm{~b}$} & $102.3(3.0)$ & $26.94(31)$ & 0.52 \\
\hline & \multicolumn{3}{|c|}{$w_{0}, \beta=1.726$ and $\beta=1.836$ only, $P_{2}=0$} \\
\hline M1a & $99.8(1.6)$ & $27.26(22)$ & 0.18 \\
\hline M2a & $100.4(1.5)$ & $27.26(22)$ & 0.18 \\
\hline M1b & $99.7(1.7)$ & $27.26(22)$ & 0.16 \\
\hline \multirow[t]{2}{*}{$\mathrm{M} 2 \mathrm{~b}$} & $100.5(1.6)$ & $27.26(22)$ & 0.17 \\
\hline & \multicolumn{3}{|c|}{$w_{0}, \beta=1.778$ and $\beta=1.836$ only, $P_{2}=0$} \\
\hline M1a & $97.7(2.8)$ & $27.54(29)$ & 0.21 \\
\hline M2a & $98.8(2.4)$ & $27.55(30)$ & 0.32 \\
\hline M1b & $97.1(2.6)$ & $27.53(29)$ & 0.28 \\
\hline $\mathrm{M} 2 \mathrm{~b}$ & $99.5(2.5)$ & $27.55(30)$ & 0.37 \\
\hline
\end{tabular}
$190 \mathrm{MeV}$ in the fourth panel, using $w_{0}$ and only the two coarser lattice spacings in the fifth panel, using $w_{0}$ and only the coarser and finest lattice spacings in the sixth panel, and using $w_{0}$ and only the two finest lattice spacings in the last panel. To determine the ratio $m_{s} / m_{u d}$, we use the values of $m_{u d}$ from Table IX.

for the specific determination of $Z_{P}$. Other determinations yield similar results.

The last step of the analysis is an interpolation using Eq. (48) to find the value of $m_{s}$ that reproduces $m_{K}^{\text {isoQCD }}=$ 494.2(3) MeV given in Eq. (27). As in the case of the pion, to estimate the systematic errors related to the scale setting, in the chiral extrapolation and the continuum limit, we repeat the analyses using two different GF scales, excluding the 
TABLE XII. Values of the bare valence quark mass parameters and the corresponding values of $a m_{D}$ from the GEVP analysis [32] for each of the ensembles used in the analysis in the $D$ meson. The number of configuration analyzed for each ensemble is the reported in Table VIII.

\begin{tabular}{lcccc}
\hline \hline Ensemble & $a \mu_{\ell}$ & $a \mu_{c}$ & $a m_{D}$ \\
\hline $\mathrm{cA} 211.53 .24$ & 0.00530 & 0.20770 .23360 .25960 .2856 & $0.7694(12) 0.8207(12) 0.8703(13) 0.9179(14)$ \\
$\mathrm{cA} 211.40 .24$ & 0.00400 & 0.20770 .23360 .25960 .2856 & $0.7676(13) 0.8190(15) 0.8686(16) 0.9164(18)$ \\
$\mathrm{cA} 211.30 .32$ & 0.00300 & 0.20770 .23360 .25960 .2856 & $0.76284(67) 0.81428(73) 0.86387(80) 0.91167(86)$ \\
$\mathrm{cA} 211.12 .48$ & 0.00120 & 0.20770 .23360 .25960 .2856 & $0.7567(17) 0.8078(20) 0.8570(23) 0.9044(26)$ \\
cB211.25.48 & 0.00250 & 0.17450 .19620 .21810 .2399 & $0.6477(11) 0.6919(12) 0.7349(12) 0.7762(13)$ \\
cB211.14.64 & 0.00140 & 0.17450 .19620 .21810 .2399 & $0.64373(70) 0.68816(79) 0.73133(87) 0.77284(96)$ \\
cB211.072.64 & 0.00072 & 0.17450 .19620 .21810 .2399 & $0.6415(13) 0.6860(15) 0.7292(16) 0.7709(18)$ \\
cC211.20.48 & 0.00200 & 0.15260 .17160 .19070 .2098 & $0.55934(60) 0.59821(64) 0.63589(68) 0.67237(73)$ \\
cC211.06.80 & 0.00060 & 0.15260 .17160 .19070 .2098 & $0.5533(11) 0.5921(12) 0.6297(13) 0.6660(15)$ \\
\hline \hline
\end{tabular}

ensembles with pion mass larger than $190 \mathrm{MeV}$ and the term proportional to $P_{2}$ in Eq. (49), and with only pairs of values of the lattice spacing. The results are shown in Table XI.

We use the same procedure as for $m_{u d}$ to obtain the mean value, statistical, and systematic errors for $m_{s}$ using Eqs. (40)-(42) excluding the analyses with $\chi^{2} /$ d.o.f. $>$ 2.5. We find in the $\overline{\mathrm{MS}}$ scheme at $2 \mathrm{GeV}$

$$
\begin{gathered}
m_{s}=101.0(1.9)_{\text {stat }}(1.3)_{\text {syst }} \mathrm{MeV}=101.0(2.3) \mathrm{MeV}, \\
\frac{m_{s}}{m_{u d}}=27.30(24)_{\text {stat }}(14)_{\text {syst }}=27.30(28)
\end{gathered}
$$

\section{Charm quark mass}

In this section, we present our determination of the mass of the charm quark obtained by analyzing $D$ - and $D_{s^{-}}$ meson masses, following a strategy similar to the one presented for the determination of $m_{s}$. For the valence mass parameters, we evaluate correlators for $\mu_{\ell}$ values equal to its sea counterpart, as well as at four values of the quark mass parameter $\mu_{c}$ in the range of the charm mass. In the case of the $D_{s}$ meson, we also use three values of the quark mass parameter $\mu_{s}$ equal to the values used in the kaon analysis (see Table $\mathrm{X}$ ). The values for the $D$-meson masses are given in Table XII, while the ones for the $D_{s}$-meson are given in Table XIII. The $D$ - and $D_{s}$-meson correlators are computed using both smeared and local interpolating fields. Using the four combinations of smeared-smeared, smearedlocal, and local-local correlators, we construct a $2 \times 2$ matrix and perform a generalized eigenvalue problem (GEVP) analysis [32] to extract the mass of the $D$ - and $D_{s}$-mesons. ${ }^{6}$ We employ Jacobi smearing for the quark

\footnotetext{
${ }^{6}$ As discussed in Ref. [32], the mass of the ground state is estimated through an average over the values in the plateau region using the principal correlator corresponding to the smallest eigenvalue $\lambda_{0}\left(t, t_{0}\right)$ obtained from the GEVP for a suitable choice of the reference time $t_{0}$. In other words, we fit $\lambda_{0}\left(t, t_{0}\right)=C\left(e^{-m_{D, D_{s}}\left(t-t_{0}\right)}+e^{-m_{D, D_{s}}\left((T-t)-t_{0}\right)}\right)$.
}

fields [33], combined with array processor experiment (APE) smearing of the gauge links [34] used in the Jacobi smearing function. The values of $m_{D}$ and $m_{D_{s}}$ used in this analysis are reported in Tables XII and XIII.

Analogously to the case of the analysis for the strange quark mass determination, we interpolate the $D$ and $D_{s}$ masses to three reference values given by $\left(m_{c} w_{0}\right)_{\text {ref }}=0.94$, 1.04, 1.08 using the Ansatz

$$
m_{D_{s}}=a+b m_{c} w_{0}
$$

For the $D_{s}$ meson, we also perform an interpolation to the mass $m_{s}$ given in Table XI. At each of the reference charm quark masses, we extrapolate to the continuum and to the isospin-symmetric QCD (isoQCD) light quark mass $m_{\ell}=m_{u d}$ using the following polynomials in $m_{\ell}$,

$$
\begin{aligned}
& m_{D}=P_{0}+P_{1} m_{\ell} w_{0}+P_{2} a^{2} / w_{0}^{2}, \\
& m_{D_{s}}=P_{0}^{s}+P_{1}^{s} m_{\ell} w_{0}+P_{2}^{s} a^{2} / w_{0}^{2},
\end{aligned}
$$

where $P_{j}$ and $P_{j}^{s}, j=0,1,2$, are fit parameters. For each reference mass $\left(m_{c} w_{0}\right)_{\text {ref }}$, we compute the masses $m_{D}$ and $m_{D_{s}}$ in the continuum limit at the isoQCD value of $m_{\ell}=$ $m_{u d}$ given in Table IX. We then perform an interpolation in $m_{c}$ with the Ansatz given in Eq. (52) to compute the value of $m_{c}$ that reproduces the isoQCD masses of the $D$ of $D_{s}$ mesons, given in Eqs. (28) and (29). We note that the analysis is done separately using either the $D$ or the $D_{s}$ meson.

The resulting fits to Eqs. (53) and (54) for the $D$ and $D_{s}$ mesons are shown in Fig. 11 for the case where $Z_{P}$ is determined from the M2b method.

The values in physical units that we obtain for the $m_{c}$ mass are shown in Table XIV.

We combine all the values given in Table XIV excluding the analyses with $\chi^{2} /$ d.o.f. $>2.5$ as in the case of the pion and kaon with Eqs. (40)-(42). We find 
TABLE XIII. Values of the bare valence quark mass parameters and the corresponding values of $a m_{D_{s}}$ from the GEVP analysis [32] for each of the ensembles used in the analysis in the $D_{s}$ meson. The strange quark masses $m_{s}$ are the same used in the kaon sector Table X. The number of configurations analyzed for each ensemble is the reported in Table VIII.

\begin{tabular}{|c|c|c|c|}
\hline Ensemble & $a \mu_{\ell}$ & $a \mu_{c}$ & $a m_{D_{s}}$ \\
\hline \multirow[t]{3}{*}{ cA211.53.24 } & 0.0176 & 0.20770 .23360 .25960 .2856 & $0.79724(78) 0.84790(81) 0.89693(85) 0.94414(88)$ \\
\hline & 0.0220 & 0.20770 .23360 .25960 .2856 & $0.80696(72) 0.85738(75) 0.90619(78) 0.95323(81)$ \\
\hline & 0.0264 & 0.20770 .23360 .25960 .2856 & $0.81656(67) 0.86673(69) 0.91534(72) 0.96220(75)$ \\
\hline \multirow[t]{3}{*}{ cA211.40.24 } & 0.0176 & 0.20770 .23360 .25960 .2856 & $0.79838(58) 0.84918(63) 0.89825(69) 0.94561(75)$ \\
\hline & 0.0220 & 0.20770 .23360 .25960 .2856 & $0.80789(50) 0.85844(54) 0.90729(59) 0.95447(64)$ \\
\hline & 0.0264 & 0.20770 .23360 .25960 .2856 & $0.81733(44) 0.86763(48) 0.91626(52) 0.96326(56)$ \\
\hline \multirow[t]{3}{*}{ cA211.30.32 } & 0.0176 & 0.20770 .23360 .25960 .2856 & $0.79610(28) 0.84675(30) 0.89567(32) 0.94289(34)$ \\
\hline & 0.0220 & 0.20770 .23360 .25960 .2856 & $0.80573(26) 0.85613(27) 0.90484(28) 0.95189(30)$ \\
\hline & 0.0264 & 0.20770 .23360 .25960 .2856 & $0.81527(24) 0.86544(25) 0.91395(26) 0.96082(27)$ \\
\hline \multirow[t]{3}{*}{ cA211.12.48 } & 0.0176 & 0.20770 .23360 .25960 .2856 & $0.79416(39) 0.84469(42) 0.89347(45) 0.94055(48)$ \\
\hline & 0.0220 & 0.20770 .23360 .25960 .2856 & $0.80385(35) 0.85417(37) 0.90278(39) 0.94973(42)$ \\
\hline & 0.0264 & 0.20770 .23360 .25960 .2856 & $0.81345(32) 0.86355(33) 0.91199(35) 0.95878(37)$ \\
\hline \multirow[t]{3}{*}{ cB211.25.48 } & 0.0148 & $\begin{array}{llll}0.1745 & 0.1962 & 0.2181 & 0.2399\end{array}$ & $0.67488(25) 0.71849(26) 0.76093(27) 0.80181(29)$ \\
\hline & 0.0185 & $\begin{array}{llll}0.1745 & 0.1962 & 0.2181 & 0.2399\end{array}$ & $0.68310(20) 0.72652(21) 0.76881(22) 0.80956(23)$ \\
\hline & 0.0222 & $\begin{array}{llll}0.1745 & 0.1962 & 0.2181 & 0.2399\end{array}$ & $0.69127(17) 0.73451(17) 0.77664(18) 0.81726(19)$ \\
\hline \multirow[t]{3}{*}{ cB211.14.64 } & 0.0148 & $\begin{array}{llll}0.1745 & 0.1962 & 0.2181 & 0.2399\end{array}$ & $0.67415(20) 0.71771(21) 0.76010(22) 0.80094(24)$ \\
\hline & 0.0185 & $\begin{array}{llll}0.1745 & 0.1962 & 0.2181 & 0.2399\end{array}$ & $0.68243(18) 0.72579(19) 0.76803(20) 0.80873(21)$ \\
\hline & 0.0222 & $\begin{array}{llll}0.1745 & 0.1962 & 0.2181 & 0.2399\end{array}$ & $0.69064(16) 0.73381(17) 0.77589(18) 0.81646(18)$ \\
\hline \multirow[t]{3}{*}{ cB211.072.64 } & 0.0148 & 0.17450 .19620 .21810 .2399 & $0.67351(22) 0.71707(24) 0.75948(27) 0.80035(31)$ \\
\hline & 0.0185 & $\begin{array}{llll}0.1745 & 0.1962 & 0.2181 & 0.2399\end{array}$ & $0.68188(19) 0.72526(21) 0.76752(23) 0.80826(26)$ \\
\hline & 0.0222 & $\begin{array}{llll}0.1745 & 0.1962 & 0.2181 & 0.2399\end{array}$ & $0.69016(17) 0.73336(18) 0.77547(21) 0.81608(23)$ \\
\hline \multirow[t]{3}{*}{ cC211.20.48 } & 0.0128 & 0.15260 .17160 .19070 .2098 & $0.58322(25) 0.62162(26) 0.65893(27) 0.69511(28)$ \\
\hline & 0.0161 & 0.15260 .17160 .19070 .2098 & $0.59050(22) 0.62874(23) 0.66592(24) 0.70199(25)$ \\
\hline & 0.0193 & 0.15260 .17160 .19070 .2098 & $0.59752(20) 0.63561(21) 0.67266(21) 0.70863(22)$ \\
\hline \multirow[t]{3}{*}{ cC211.06.80 } & 0.0128 & 0.15260 .17160 .19070 .2098 & $0.58181(21) 0.62019(23) 0.65748(25) 0.69365(28)$ \\
\hline & 0.0161 & 0.15260 .17160 .19070 .2098 & $0.58919(19) 0.62741(20) 0.66457(22) 0.70063(24)$ \\
\hline & 0.0193 & 0.15260 .17160 .19070 .2098 & $0.59629(17) 0.63436(18) 0.67139(20) 0.70734(21)$ \\
\hline
\end{tabular}
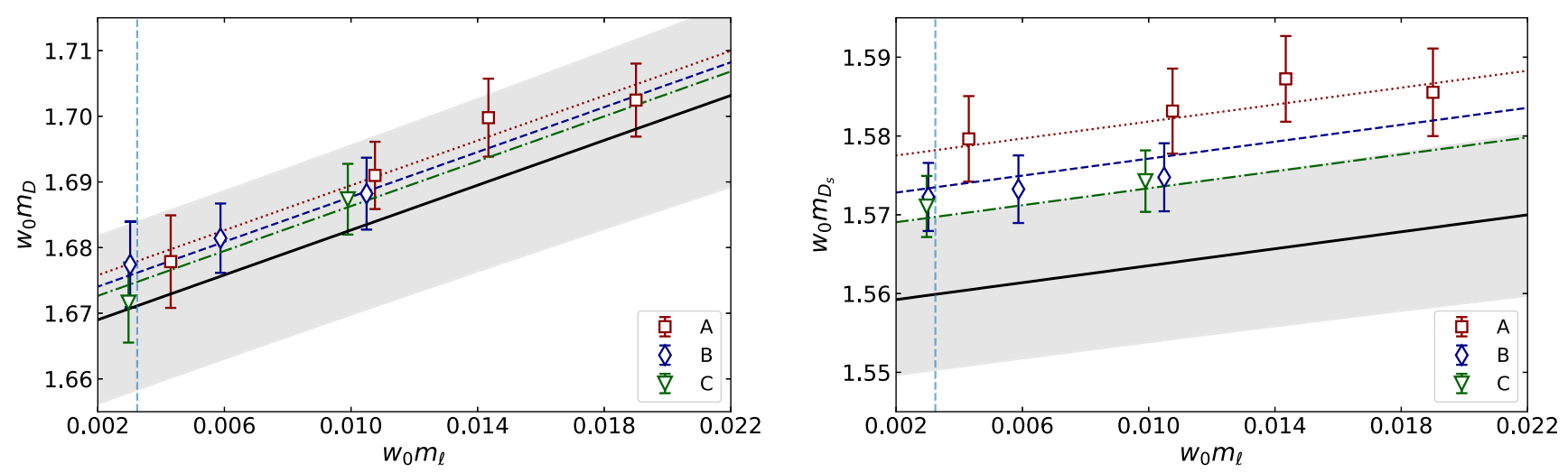

FIG. 11. Results obtained by fitting to Eq. (53) for the $D$ meson (left panel) and Eq. (54) for the $D_{s}$ meson (right panel) using the value of $Z_{P}$ extracted from the $\mathrm{M} 2 \mathrm{~b}$ method and using $w_{0}$ to set the scale. The notation is the same as that of Fig. 10. 
TABLE XIV. Values of the charm quark mass $m_{c}$ in the $\overline{\mathrm{MS}}$ scheme at $3 \mathrm{GeV}$ and the ratio $m_{c} / m_{s}$ from the analysis of the $D$ and $D_{s}$ meson, for different determinations of $Z_{P}$ and GF scales. Results using the GF scale $w_{0}$ and all the ensembles of Table XII are given in the top most panel, using $t_{0} / w_{0}$ in the second panel, using $\sqrt{t_{0}}$ in the third panel, using $w_{0}$ and limiting $m_{\pi}<190 \mathrm{MeV}$ in the fourth panel, using $w_{0}$ and only the two coarser lattice spacings in the fifth panel, using $w_{0}$ and only the coarser and finest lattice spacings in the sixth panel, and using $w_{0}$ and only the two finest lattice spacings in the last panel. To determine the ratio $m_{c} / m_{s}$ we use the values of $m_{s}$ from Table XI.

\begin{tabular}{|c|c|c|c|c|c|c|}
\hline \multirow[b]{2}{*}{$Z_{P}$} & \multicolumn{3}{|c|}{$D$} & \multicolumn{3}{|c|}{$D_{s}$} \\
\hline & $m_{c}(\mathrm{MeV})$ & $m_{c} / m_{s}$ & $\chi^{2} /$ d.o.f. & $m_{c}(\mathrm{MeV})$ & $m_{c} / m_{s}$ & $\chi^{2} /$ d.o.f. \\
\hline M1a & 1041(14) & $11.504(76)$ & 0.25 & $1039(13)$ & $11.476(70)$ & 0.62 \\
\hline $\mathrm{M} 2 \mathrm{a}$ & 1041(13) & $11.443(76)$ & 0.16 & $1038(12)$ & $11.414(69)$ & 0.45 \\
\hline M1b & $1043(14)$ & $11.496(77)$ & 0.45 & 1040(13) & $11.463(71)$ & 1.2 \\
\hline $\mathrm{M} 2 \mathrm{~b}$ & 1039(13) & $11.418(78)$ & 0.14 & $1037(12)$ & $11.397(71)$ & 0.21 \\
\hline $\begin{array}{l}\text { M1a } \\
\text { M2a } \\
\text { M1b } \\
\text { M2b }\end{array}$ & $\begin{array}{l}1042(15) \\
1045(14) \\
1043(15) \\
1045(14)\end{array}$ & $\begin{array}{l}11.385(84) \\
11.283(85) \\
11.375(86) \\
11.248(86)\end{array}$ & $\begin{array}{l}0.12 \\
0.15 \\
0.2 \\
0.25\end{array}$ & $\begin{array}{l}1038(13) \\
1041(12) \\
1039(14) \\
1041(13)\end{array}$ & $\begin{array}{l}11.342(79) \\
11.235(78) \\
11.322(81) \\
11.206(80)\end{array}$ & $\begin{array}{l}0.36 \\
0.25 \\
0.76 \\
0.1\end{array}$ \\
\hline $\begin{array}{l}\text { M1a } \\
\text { M2a } \\
\text { M1b } \\
\text { M2b }\end{array}$ & $\begin{array}{l}1041(14) \\
1043(13) \\
1043(14) \\
1042(13)\end{array}$ & $\begin{array}{l}11.448(79) \\
11.368(80) \\
11.439(81) \\
11.338(81)\end{array}$ & $\begin{array}{l}0.16 \\
0.13 \\
0.29 \\
0.18\end{array}$ & $\begin{array}{l}1038(13) \\
1039(12) \\
1039(14) \\
1039(12)\end{array}$ & $\begin{array}{l}11.412(74) \\
11.329(73) \\
11.396(75) \\
11.306(75)\end{array}$ & $\begin{array}{l}0.48 \\
0.35 \\
0.96 \\
0.15\end{array}$ \\
\hline $\begin{array}{l}\text { M1a } \\
\text { M2a } \\
\text { M1b } \\
\text { M2b }\end{array}$ & $\begin{array}{l}1042(16) \\
1041(15) \\
1043(16) \\
1039(15)\end{array}$ & $\begin{array}{l}11.46(14) \\
11.39(14) \\
11.45(14) \\
11.37(14)\end{array}$ & $\begin{array}{c}w_{0}, m_{\pi}< \\
0.099 \\
0.028 \\
0.38 \\
0.25\end{array}$ & $\begin{array}{l}V, P_{2}=0 \\
1037(13) \\
1035(12) \\
1037(14) \\
1034(12)\end{array}$ & $\begin{array}{l}11.396(96) \\
11.333(97) \\
11.380(96) \\
11.316(98)\end{array}$ & $\begin{array}{l}1.3 \\
0.8 \\
2.6 \\
0.26\end{array}$ \\
\hline $\begin{array}{l}\text { M1a } \\
\text { M2a } \\
\text { M1b } \\
\text { M2b }\end{array}$ & $\begin{array}{l}1057(31) \\
1046(26) \\
1063(28) \\
1037(24)\end{array}$ & $\begin{array}{l}11.34(16) \\
11.26(16) \\
11.34(16) \\
11.25(16)\end{array}$ & $\begin{aligned} w_{0}, \beta= & 1.726 \text { ar } \\
& 0.12 \\
& 0.13 \\
& 0.11 \\
& 0.13\end{aligned}$ & $\begin{array}{l}778 \text { only, } P_{2} \\
1062(29) \\
1052(24) \\
1069(26) \\
1042(22)\end{array}$ & $\begin{array}{l}11.41(15) \\
11.32(15) \\
11.40(15) \\
11.31(15)\end{array}$ & $\begin{array}{l}0.043 \\
0.045 \\
0.04 \\
0.046\end{array}$ \\
\hline $\begin{array}{l}\text { M1a } \\
\text { M2a } \\
\text { M1b } \\
\text { M2b }\end{array}$ & $\begin{array}{l}1038(14) \\
1039(13) \\
1037(14) \\
1039(13)\end{array}$ & $\begin{array}{l}11.545(76) \\
11.487(77) \\
11.546(76) \\
11.474(77)\end{array}$ & $\begin{aligned} w_{0}, \beta= & 1.726 \text { ar } \\
& 0.23 \\
& 0.24 \\
& 0.21 \\
& 0.23\end{aligned}$ & $\begin{array}{c}836 \text { only, } P_{2} \\
1036(13) \\
1036(12) \\
1035(14) \\
1036(12)\end{array}$ & $\begin{array}{l}11.522(66) \\
11.456(67) \\
11.523(66) \\
11.441(68)\end{array}$ & $\begin{array}{l}0.097 \\
0.096 \\
0.086 \\
0.095\end{array}$ \\
\hline $\begin{array}{l}\text { M1a } \\
\text { M2a } \\
\text { M1b } \\
\text { M2b }\end{array}$ & $\begin{array}{l}1029(28) \\
1037(24) \\
1022(26) \\
1044(24)\end{array}$ & $\begin{array}{l}11.68(12) \\
11.65(12) \\
11.68(12) \\
11.64(12)\end{array}$ & $\begin{aligned} w_{0}, \beta= & 1.778 \text { ar } \\
& 0.082 \\
& 0.12 \\
& 0.097 \\
& 0.13\end{aligned}$ & $\begin{array}{c}836 \text { only, } P_{2} \\
1019(25) \\
1027(22) \\
1014(23) \\
1033(21)\end{array}$ & $\begin{array}{l}11.576(87) \\
11.534(88) \\
11.585(86) \\
11.520(89)\end{array}$ & $\begin{array}{l}0.0033 \\
0.0045 \\
0.0045 \\
0.005\end{array}$ \\
\hline
\end{tabular}

$m_{c}=1039(15)_{\text {stat }}(6)_{\text {syst }} \mathrm{MeV}=1039(16) \mathrm{MeV}$

$$
\frac{m_{c}}{m_{s}}=11.43(9)_{\text {stat }}(10)_{\text {syst }}=11.43(13)
$$

where the charm quark mass is given in the $\overline{\mathrm{MS}}$ at $3 \mathrm{GeV}$.

\section{BARYON SECTOR ANALYSIS}

In the baryon sector, we use the nucleon and pion masses to set the scale and determine the light quark mass. We use the $\Omega^{-}(s s s)$ and the $\Lambda_{c}(u d c)$ masses to determine, respectively, the strange and charm quark masses. The range of validity of ChPT in the baryon sector is more limited as compared to that in the pion sector, and thus we restrict ourselves to using pion masses up to $260 \mathrm{MeV}$.

\section{A. Methodology}

In order to compute the baryon masses, we construct the following two-point correlation functions at zero momentum, defined as 
$C_{B}^{ \pm}(t)=\sum_{\vec{x}}\left\langle 0\left|\frac{1}{4} \operatorname{Tr}\left[\left(1 \pm \gamma_{0}\right) J_{B\left(q q^{\prime} q^{\prime \prime}\right)}(\vec{x}, t) \bar{J}_{B\left(q q^{\prime} q^{\prime \prime}\right)}(\overrightarrow{0}, 0)\right]\right| 0\right\rangle$,

where $J_{B\left(q q^{\prime} q^{\prime \prime}\right)}$ is the interpolating operator for the baryon $B\left(q, q^{\prime}, q^{\prime \prime}\right)$ with $q, q^{\prime}$, and $q^{\prime \prime} \in\{l, s, c\}$. In this work, we increase statistics by considering both $\frac{1}{2}\left(1 \pm \gamma_{0}\right)$ projectors. For the interpolating fields of the nucleon, the $\Omega$, and the $\Lambda_{c}$ we take, respectively,

$$
\begin{aligned}
J_{N} & =\epsilon^{a b c}\left(u_{a}^{T} C \gamma_{5} d_{b}\right) u_{c}, \\
J_{\Omega} & =\epsilon^{a b c}\left(s_{a} C \gamma_{\mu} s_{b}\right) s_{c}, \\
J_{\Lambda_{c}} & =\frac{1}{\sqrt{6}} \epsilon^{a b c}\left[2\left(u_{a}^{T} C \gamma_{5} d_{b}\right) c_{c}+\left(u_{a}^{T} C \gamma_{5} c_{a}\right) d_{c}\right. \\
& \left.-\left(d_{a}^{T} C \gamma_{5} c_{b}\right) u_{c}\right],
\end{aligned}
$$

where latin indices refer to color, $\epsilon^{a b c}$ is the antisymmetric tensor, and $C$ is the charge conjugation matrix.

In order to suppress contributions from excited states, we apply Gaussian smearing to each quark field $q(\vec{x}, t)$. The smeared quark field is given by $q_{\text {smear }}(\vec{x}, t)=\sum_{\vec{y}} F(\vec{x}, \vec{y}$; $U(t)) q(\vec{y}, t)$, where $F$ is the gauge-invariant smearing function

$$
F(\vec{x}, \vec{y} ; U(t))=(1+\alpha H)^{n}(\vec{x}, \vec{y} ; U(t)),
$$

constructed from the hopping matrix understood as a matrix in coordinate, color, and spin space,

$$
H(\vec{x}, \vec{y}, U(t))=\sum_{i=1}^{3}\left(U_{i}(\vec{x}, t) \delta_{\vec{x}, \vec{y}-a \hat{i}}+U^{\dagger}(\vec{x}-a \hat{i}, t) \delta_{\vec{x}, \vec{y}+a \hat{i}}\right) .
$$

In addition, we apply APE smearing to the spatial links that enter the hopping matrix $H$. Different Gaussian smearing is applied to the light and strange quarks. The parameters of the Gaussian and APE smearing for each ensemble for the light and strange quarks are given in Table XV. The charm quark interpolating fields are not smeared.

Two-point correlators for the $\Omega$ and $\Lambda_{c}$ are computed for each ensemble at three different values of the valence strange and charm quark masses $a \mu_{s}$ and $a \mu_{c}$. For each value, an analysis of the two-point correlator is carried out in order to determine the masses $m_{\Omega}$ and $m_{\Lambda_{c}}$ as a function of $\mu_{s}$ and $\mu_{c}$, respectively. The effective mass

$$
a m_{B}^{\mathrm{eff}}=\log \left(\frac{C_{B}(t)}{C_{B}(t+a)}\right)
$$

can be written using the spectral decomposition of the twopoint correlators as
TABLE XV. Parameters $n$ and $\alpha$ entering the Gaussian smearing in Eq. (59) per ensemble for the light and strange quark interpolating fields. The parameters for the APE smearing are kept the same for all ensembles. They are $n_{\mathrm{APE}}=50$ and $\alpha_{\mathrm{APE}}=0.5$.

\begin{tabular}{lrrrrr}
\hline \hline & \multicolumn{2}{c}{ Light } & & \multicolumn{2}{c}{ Strange } \\
\cline { 2 - 3 } \cline { 5 - 6 } Ensemble & $n$ & & & $n$ & $\alpha$ \\
\hline cA211.30.32 & 40 & 1.0 & & 25 & 4.0 \\
cA211.12.48 & 50 & 1.0 & 25 & 4.0 \\
cB211.25.32 & 40 & 1.0 & 25 & 4.0 \\
cB211.25.48 & 40 & 1.0 & 25 & 4.0 \\
cB211.14.64 & 70 & 1.0 & 25 & 4.0 \\
cB211.072.64 & 125 & 0.4 & 25 & 4.0 \\
cC211.20.48 & 40 & 1.0 & & 25 & 1.0 \\
cC211.06.80 & 140 & 1.0 & 25 & 1.0 \\
\hline \hline
\end{tabular}

$$
a m_{B}^{\mathrm{eff}} \approx a m_{B}+\log \left(\frac{1+\sum_{j=1}^{K} c_{j} e^{-\Delta_{j} t}}{1+\sum_{j=1}^{K} c_{j} e^{-\Delta_{j}(t+a)}}\right),
$$

where $\Delta_{j}$ is the mass difference of the $j$ th excited state with respect to the ground state mass $m_{B}$. We consider one-, two-, and three-state fits by taking $K=0,1,2$ in Eq. (62). This allows us to check the consistency in our determination of the ground state mass $m_{B}$. Since statistical errors are larger for baryons as compared to those of mesons and grow rapidly with increasing time separation $t$, it is important to identify the ground state for as small a time separation as possible, so that we can be confident that excited are sufficiently suppressed. Our procedure for identifying $m_{B}$ is as follows:

(1) We keep the upper time used in the fit constant. The upper time is chosen so that statistical errors are reliably evaluated.

(2) We fit the effective mass keeping two excited states; i.e., we take $K=2$ in Eq. (62) and vary the lower time used in the fit $t_{\text {low }}^{3 s t} / a$ from 1 to 3 . We choose the parameters of the fit that has the smallest $t_{\text {low }}^{3 s t}$ for which $\chi^{2} /$ d.o.f. $\lesssim 1$. This determines $m_{B}^{3 s t}$.

(3) We then fit the effective mass including one excited state; i.e., we set $K=1$ in Eq. (62) and vary $t_{\text {low }}^{2 s t}$ for $t_{\text {low }}^{2 s t}>t_{\text {low }}^{3 s t}$ until the extracted mass $m_{B}^{2 s t}$ satisfies the criterion $\left|m_{B}^{2 s t}-m_{B}^{3 s t}\right|<\delta m_{B}^{2 s t}$, where $\delta m_{B}^{2 s t}$ is the statistical error on $m_{B}^{2 s t}$ (i.e., the difference in the central values of the baryon masses extracted using one and two excited states are compatible within the statistical error of $m_{B}^{2 s t}$ ).

(4) Having determined $m_{B}^{2 s t}$, we make a single state fit; i.e., we set $K=0$ in Eq. (62) and vary the lower value of $t$. We choose $t_{\text {low }}^{1 s t}>t_{\text {low }}^{2 s t}$ and take the smallest value that satisfies $\left|m_{B}^{1 s t}-m_{B}^{2 s t}\right|<\delta m_{B}^{1 s t}$, where $\delta m_{B}^{1 s t}$ is the statistical error on $m_{B}^{1 s t}$, provided $\chi^{2} /$ d.o.f. $\sim 1$. We used $m_{B}^{1 s t}$ as our final value for $m_{B}$. 

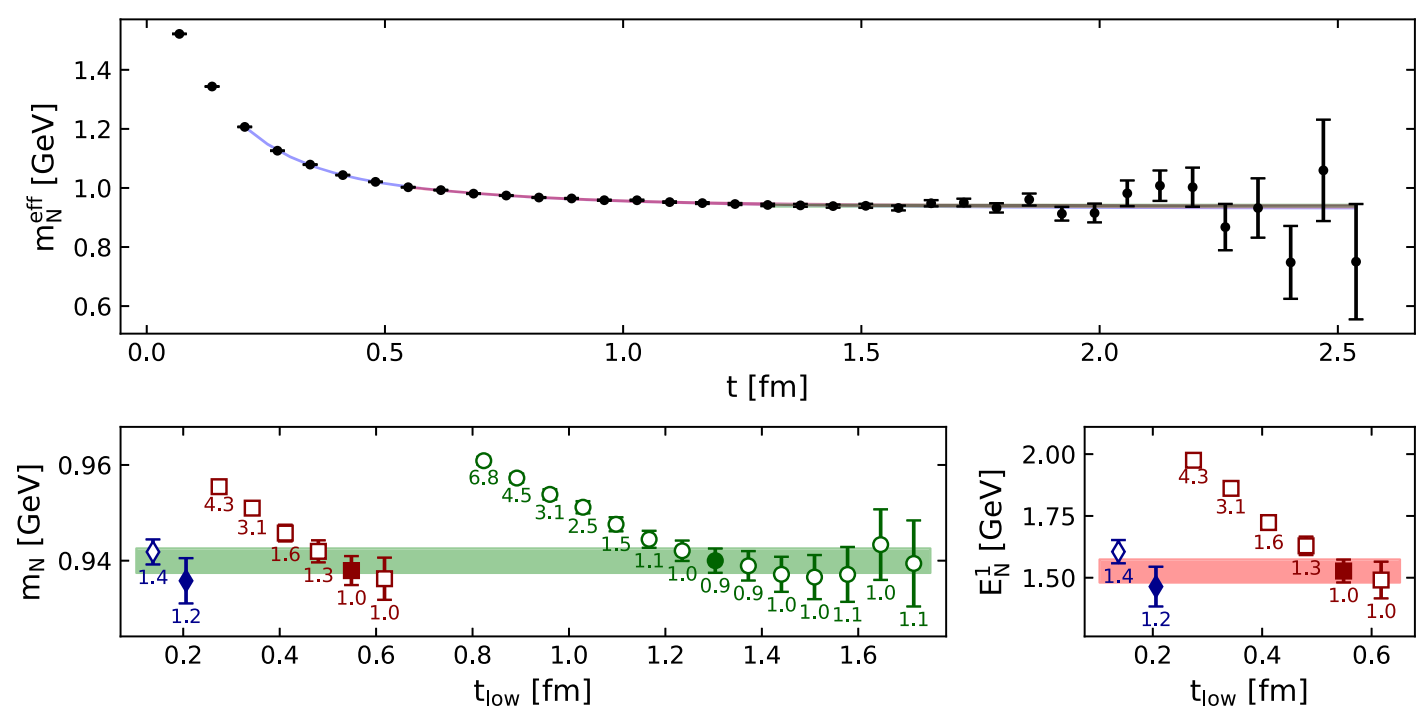

FIG. 12. Upper panel: we show the nucleon effective mass $m_{N}^{\text {eff }}(t)$ as a function of $t$ for the cC211.06.80 ensemble. Lower left panel: we show the convergence of the extracted value of $m_{N}$ as a function of the lowest time $t_{\text {low }}$ used in the fit when we include one state in the fit (green open circles), when we include two states (open red squares) and when we include three states (open blue rhombus). Lower right panel: the same as the lower left panel but for the values extracted for the mass of the first excited state. The filled symbols and green and red bands show the values we pick for $m_{N}$ and for the mass of the excited state $m_{\text {Roper }}$, respectively. For each point, we give the $\chi^{2} /$ d.o.f. of the fit.

We illustrate our analysis for the extraction of the masses by giving representative examples for the nucleon, $\Omega^{-}$and $\Lambda_{c}$. In all cases, we use correlation functions with smeared sources and for the ensembles listed in Table XVI. In Fig. 12, we show an example of the results obtained using the nucleon correlators for the $\mathrm{cC} 211.06 .80$ ensemble, and in Table XVI, we give the number of configurations and source positions used, the fit ranges for the one-, two-, and three-state fits, as well as the extracted nucleon mass and the $\chi^{2} /$ d.o.f. As can be seen, the mass of the first excited state converges to a value compatible with the mass of the Roper for the physical point ensembles.
The nucleon-pion state, although it has lower energy, it is volume suppressed. In our two-state fits to extract the energy of the first excited state, we find that the coefficient of the second exponential compared to that of the ground state is of order 1 . This is to be contrasted with the chiral perturbation theory analysis of Ref. [35], which predicts a few percent for two-particle states. This indicates that the contribution of two particles is suppressed.

We analyze in a similar way the effective mass defined by the $\Omega$ correlator given in Eq. (57). In Fig. 13, we show an example of the effective mass $m_{\Omega}^{\text {eff }}$ for the $\mathrm{cB} 211.072 .64$ ensemble at $\mu_{s}=0.017,0.0195$, and 0.022 . As can be seen,

TABLE XVI. We give for each ensemble the resulting values of $a m_{N}$, when using one-state (fourth main column), two-state (fifth main column), and three-state (sixth main column) fits, $\bar{\chi}^{2} \equiv \chi^{2} /$ d.o.f. is the reduced $\chi^{2}, n_{\text {conf }}$ is the number of configurations analyzed, $n_{\text {srcs }}$ is the number of two-point functions generated per configuration at different source positions, and $\left[t_{\text {low }}, t_{\text {max }}\right]$ is the fitting range. We also show the values for the pion mass computed on the same statistics, $a m_{\pi}$, noticing that they are compatible with those given in Table I.

\begin{tabular}{|c|c|c|c|c|c|c|c|c|c|c|c|c|c|}
\hline \multirow[b]{2}{*}{ Ensemble } & \multirow[b]{2}{*}{$a m_{\pi}$} & \multirow[b]{2}{*}{$n_{\text {conf }}$} & \multirow[b]{2}{*}{$n_{\text {srcs }}$} & \multirow[b]{2}{*}{$t_{\max } / a$} & \multicolumn{3}{|c|}{ One-state fit } & \multicolumn{3}{|c|}{ Two-state fit } & \multicolumn{3}{|c|}{ Three-state fit } \\
\hline & & & & & $t_{\text {low }} / a$ & $\bar{\chi}^{2}$ & $a m_{N}$ & $t_{\text {low }} / a$ & $\bar{\chi}^{2}$ & $a m_{N}$ & $t_{\text {low }} / a$ & $\bar{\chi}^{2}$ & $a m_{N}$ \\
\hline cA211.30.32 & $0.12525(13)$ & 287 & 121 & 25 & 12 & 0.9 & $0.5075(18)$ & 4 & 0.8 & $0.5077(16)$ & 2 & 0.8 & $0.5068(22)$ \\
\hline cA211.12.48 & $0.080281(75)$ & 325 & 160 & 27 & 15 & 1.1 & $0.4566(22)$ & 7 & 1.4 & $0.4561(33)$ & 2 & 1.4 & $0.4589(23)$ \\
\hline cB211.25.32 & $0.10521(21)$ & 395 & 121 & 27 & 16 & 0.5 & $0.4325(38)$ & 7 & 0.5 & $0.4288(39)$ & 2 & 0.8 & $0.4301(47)$ \\
\hline cB211.25.48 & $0.104408(59)$ & 281 & 128 & 33 & 17 & 0.7 & $0.4307(18)$ & 5 & 1.5 & $0.43097(94)$ & 2 & 1.7 & $0.4305(11)$ \\
\hline cB211.14.64 & $0.078429(38)$ & 194 & 128 & 33 & 18 & 2.4 & $0.4015(28)$ & 7 & 1.7 & $0.3977(33)$ & 2 & 1.8 & $0.3990(42)$ \\
\hline cB211.072.64 & $0.056578(20)$ & 751 & 264 & 34 & 18 & 1.4 & $0.3822(17)$ & 7 & 1.1 & $0.3813(15)$ & 2 & 1.3 & $0.3823(16)$ \\
\hline cC211.20.48 & $0.086098(86)$ & 205 & 121 & 33 & 17 & 1.2 & $0.3664(17)$ & 8 & 1.4 & $0.3669(20)$ & 2 & 1.2 & $0.3658(18)$ \\
\hline cC211.06.80 & $0.047248(19)$ & 401 & 650 & 38 & 19 & 0.9 & $0.32679(88)$ & 8 & 1.0 & $0.3261(11)$ & 3 & 1.2 & $0.3253(16)$ \\
\hline
\end{tabular}



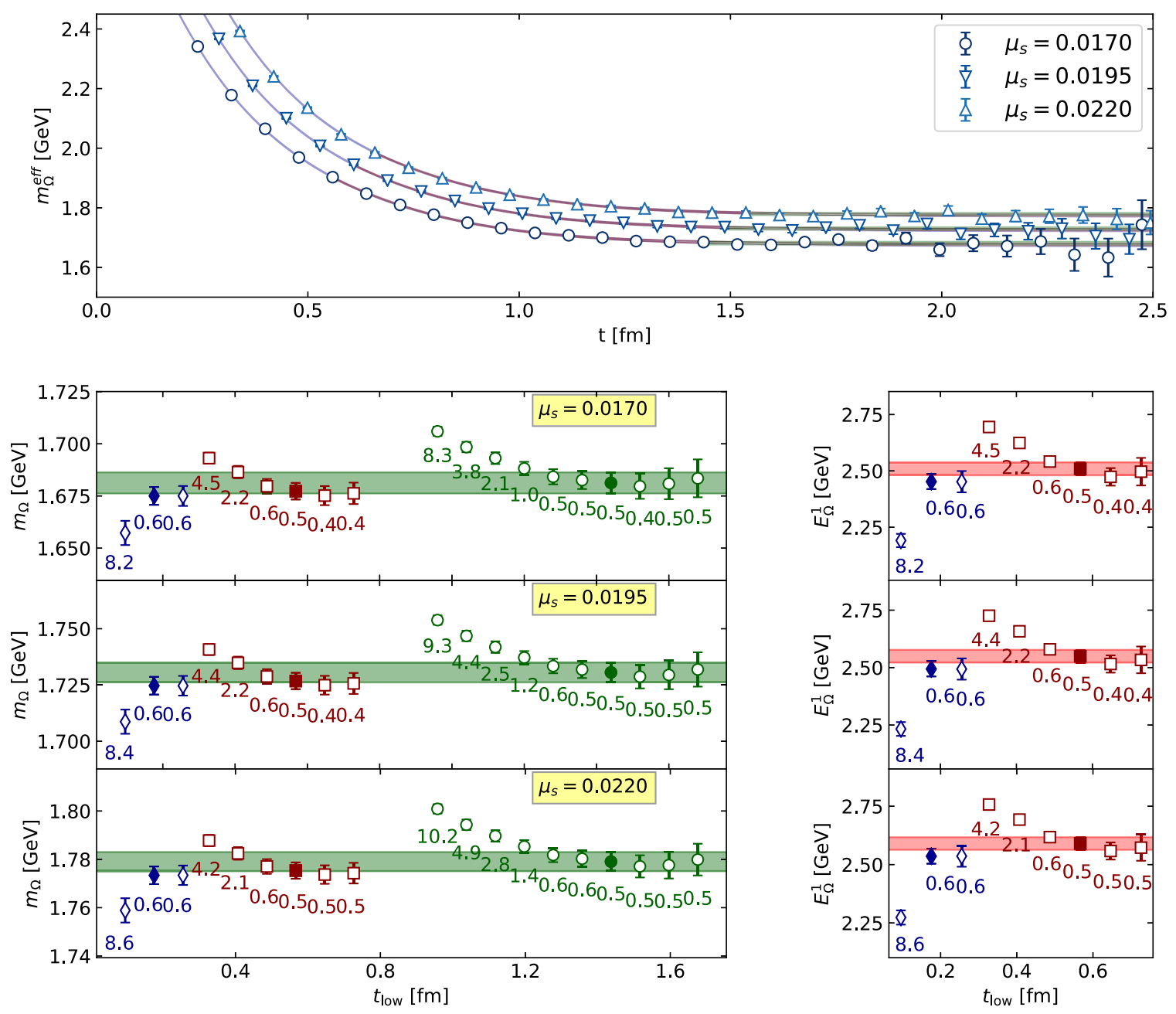

FIG. 13. The same as in Fig. 12 but for the case of $\Omega^{-}$using the cB211.072.64 ensemble for the three values of $\mu_{s}$ given the in the figure legend.

we obtain accurate results that allow us to perform a fit including up to the second excited state. We fix the maximum time for these fits to be $t_{\max } / a=34$. The convergence of the effective mass for $\Omega^{-}$as we vary $t_{\text {low }}$ is demonstrated when using one-, two-, and three-state fits. In a similar manner, the convergence of the first excited energy $E_{\Omega}^{1}$ is demonstrated by varying $t_{\text {low }}$. We employ the criterion described above to choose the value of $m_{\Omega}$ from the one-state fit at each $\mu_{s}$. We note that for all the three values of $\mu_{s}$ we find the same $t_{\text {low }}$. The masses extracted are given in Table XVII, where we also quote the reduced $-\chi^{2}$, $\bar{\chi}^{2} \equiv \chi^{2} /$ d.o.f., of the various fits.

The analysis of the two-point correlator for the $\Lambda_{c}$ proceeds in an analogous manner. We illustrate the results for the cB211.072.64 ensemble in Fig. 14 for two different values of the charm mass parameter $\mu_{c}$. From the study of the $\Omega^{-}$mass, we find that there is strong correlation among the data for the three values of $\mu_{s}$ as demonstrated in Fig. 16, and, thus, for $\Lambda_{c}$, we opt to use two different values of $\mu_{c}$ in the interpolation. Since $\Lambda_{c}$ is heavier and decays faster, a three-state fit is not possible, and we limit ourselves to comparing one- and two-state fits. The masses extracted, the statistics used, and the value of $\bar{\chi}^{2}$ are given in Table XVIII.

In what follows, we will use the values of $m_{N}, m_{\Omega}$, and $m_{\Lambda_{c}}$ extracted from the one-state fit given in Tables XVI-XVIII, respectively, to determine the light, strange, and charm quark masses. In order to estimate the systematic error due to the fit range, we will also use the values for the masses extracted from the one-state fit at $t_{\text {low }} / a+1$.

\section{B. Light quark mass}

We use the ChPT expression of Eq. (5) to extrapolate to the physical point. To one-loop order in ChPT [up to which the nucleon mass is expanded in Eq. (5)], we can substitute the pion mass by $m_{\pi}^{2}=2 B m_{u d}\left(1+c_{2} a^{2}\right)$ to obtain the expansion 
TABLE XVII. We give the mass of $\Omega^{-}$in lattice units using one- (fourth main column that includes $t_{\text {low }} / a$ and the reduced $\bar{\chi}^{2}$ ), two(fifth main column), and three- (sixth main column) state fits to the effective mass. In the second main column, we give the number of configurations $n_{\text {conf }}$, the number of two-point function per configuration $n_{\text {srcs }}$, and $t_{\max } / a$. In the last main column, we give the fit parameters $A_{\Omega}$ in lattice units and $B_{\Omega}$ defined in Eq. (65) using the $\Omega^{-}$mass from the one-state fit.

\begin{tabular}{|c|c|c|c|c|c|c|c|c|c|c|c|c|c|c|c|}
\hline \multirow[b]{2}{*}{ Ensemble } & \multirow[b]{2}{*}{$n_{\text {conf }}$} & \multirow[b]{2}{*}{$n_{\text {srcs }}$} & \multirow[b]{2}{*}{$t_{\max } / a$} & \multirow[b]{2}{*}{$a \mu_{s}$} & \multicolumn{3}{|c|}{ One-state fit } & \multicolumn{3}{|c|}{ Two-state fit } & \multicolumn{3}{|c|}{ Three-state fit } & \multirow[b]{2}{*}{$a A_{\Omega}$} & \multirow[b]{2}{*}{$B_{\Omega} / Z_{P}$} \\
\hline & & & & & $t_{\text {low }} / a$ & $\bar{\chi}^{2}$ & $a m_{\Omega}$ & $t_{\text {low }} / a$ & $\bar{\chi}^{2}$ & $a m_{\Omega}$ & $t_{\text {low }} / a$ & $\bar{\chi}^{2}$ & $a m_{\Omega}$ & & \\
\hline \multirow[t]{3}{*}{ cA211.30.32 } & 1260 & 16 & 28 & 0.0182 & 14 & 1.0 & $0.7890(22)$ & 4 & 1.1 & $0.7874(19)$ & 2 & 1.1 & $0.7859(30)$ & $0.645(4)$ & $7.89(11)$ \\
\hline & & & & 0.0227 & 14 & 1.3 & $0.8252(18)$ & 4 & 1.2 & $0.8237(17)$ & 2 & 0.8 & $0.8230(22)$ & & \\
\hline & & & & 0.0273 & 14 & 1.2 & $0.8610(15)$ & 4 & 1.1 & $0.8595(15)$ & 2 & 0.9 & $0.8590(19)$ & & \\
\hline \multirow[t]{3}{*}{ cA211.12.48 } & 341 & 64 & 29 & 0.0182 & 16 & 0.4 & $0.7797(27)$ & 4 & 0.6 & $0.7796(15)$ & 2 & 0.5 & $0.7783(25)$ & $0.634(5)$ & $8.00(13)$ \\
\hline & & & & 0.0227 & 16 & 0.5 & $0.8164(21)$ & 4 & 0.6 & $0.8164(13)$ & 2 & 0.6 & $0.8155(21)$ & & \\
\hline & & & & 0.0273 & 16 & 0.6 & $0.8527(17)$ & 4 & 0.6 & $0.8527(11)$ & 2 & 0.6 & $0.8521(17)$ & & \\
\hline \multirow[t]{3}{*}{ cB211.25.32 } & 492 & 16 & 28 & 0.0148 & 16 & 0.9 & $0.6684(36)$ & 8 & 1.2 & $0.6652(66)$ & 2 & 1.0 & $0.6630(86)$ & $0.551(7)$ & $7.94(23)$ \\
\hline & & & & 0.0185 & 16 & 0.9 & $0.6986(28)$ & 8 & 1.1 & $0.6959(46)$ & 2 & 0.9 & $0.6942(55)$ & & \\
\hline & & & & 0.0222 & 16 & 0.8 & $0.7274(23)$ & 8 & 1.0 & $0.7250(37)$ & 2 & 0.9 & $0.7236(42)$ & & \\
\hline \multirow[t]{3}{*}{ cB211.25.48 } & 651 & 32 & 34 & 0.0148 & 18 & 1.6 & $0.6666(21)$ & 6 & 1.0 & $0.6656(17)$ & 2 & 1.1 & $0.6649(24)$ & $0.551(4)$ & $7.79(13)$ \\
\hline & & & & 0.0185 & 18 & 1.4 & $0.6959(16)$ & 6 & 0.9 & $0.6947(14)$ & 2 & 0.9 & $0.6939(22)$ & & \\
\hline & & & & 0.0222 & 18 & 1.2 & $0.7244(13)$ & 6 & 0.8 & $0.7232(13)$ & 2 & 0.9 & $0.7224(20)$ & & \\
\hline \multirow[t]{3}{*}{ cB211.14.64 } & 446 & 16 & 32 & 0.0148 & 18 & 0.4 & $0.6603(35)$ & 6 & 0.8 & $0.6583(31)$ & 2 & 0.7 & $0.6528(63)$ & $0.542(6)$ & $8.00(21)$ \\
\hline & & & & 0.0185 & 18 & 0.5 & $0.6905(28)$ & 6 & 0.8 & $0.6883(27)$ & 2 & 0.7 & $0.6838(53)$ & & \\
\hline & & & & 0.0222 & 18 & 0.5 & $0.7197(23)$ & 6 & 0.8 & $0.7174(25)$ & 2 & 0.7 & $0.7136(46)$ & & \\
\hline \multirow[t]{3}{*}{ cB211.072.64 } & 770 & 32 & 34 & 0.0170 & 18 & 0.5 & $0.6799(20)$ & 7 & 0.5 & $0.6783(16)$ & 2 & 0.6 & $0.6774(17)$ & $0.545(4)$ & $7.90(12)$ \\
\hline & & & & 0.0195 & 18 & 0.5 & $0.6998(17)$ & 7 & 0.5 & $0.6983(15)$ & 2 & 0.6 & $0.6974(16)$ & & \\
\hline & & & & 0.0220 & 18 & 0.5 & $0.7194(16)$ & 7 & 0.5 & $0.7179(14)$ & 2 & 0.6 & $0.7171(15)$ & & \\
\hline \multirow[t]{2}{*}{ cC211.20.48 } & 205 & 13 & 34 & 0.0150 & 17 & 0.7 & $0.5906(30)$ & 6 & 0.9 & $0.5888(30)$ & 2 & 0.7 & $0.5863(32)$ & $0.474(5)$ & $7.76(19)$ \\
\hline & & & & 0.0170 & 17 & 0.8 & $0.6062(28)$ & 6 & 0.9 & $0.6043(28)$ & 2 & 0.7 & $0.6021(29)$ & & \\
\hline \multirow[t]{3}{*}{ cC211.06.80 } & 401 & 16 & 39 & 0.0150 & 20 & 0.6 & $0.5766(14)$ & 5 & 0.6 & $0.5759(10)$ & 2 & 0.7 & $0.5756(12)$ & $0.457(3)$ & $7.93(1)$ \\
\hline & & & & 0.0170 & 20 & 0.6 & $0.5926(12)$ & 5 & 0.6 & $0.5919(9)$ & 2 & 0.7 & $0.5917(11)$ & & \\
\hline & & & & 0.0190 & 20 & 0.7 & $0.6083(11)$ & 5 & 0.6 & $0.6077(9)$ & 2 & 0.7 & $0.6075(10)$ & & \\
\hline
\end{tabular}
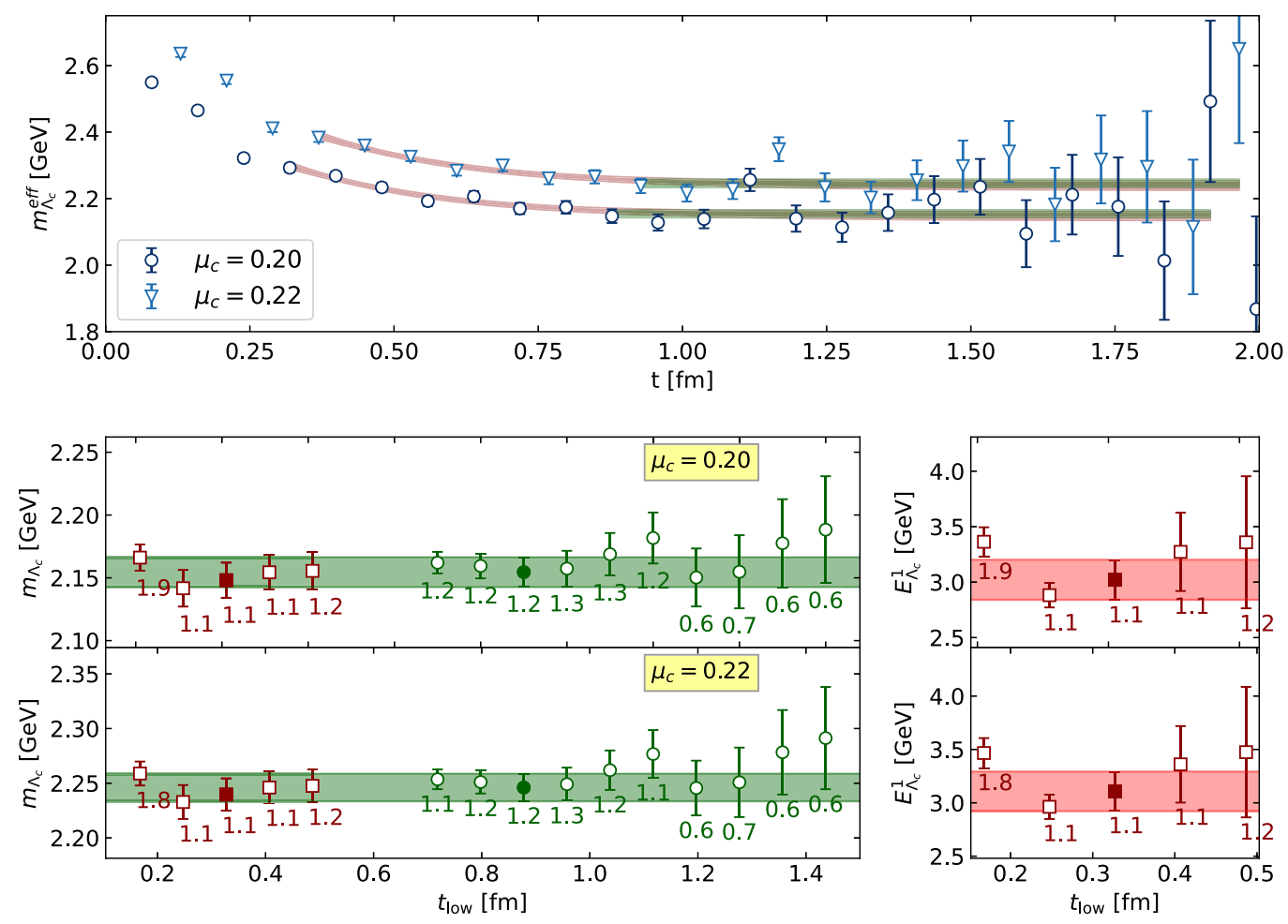

FIG. 14. The same as in Fig. 12 but for the case of $\Lambda_{c}$ using the cB211.072.64 ensemble for the two values of $\mu_{c}$ given in the figure legend. 
TABLE XVIII. The values of $m_{\Lambda_{c}}\left(\mu_{c}\right)$ mass, statistics used and fit parameters defined in Eq. (65) using a similar notation as that in Table XVII.

\begin{tabular}{|c|c|c|c|c|c|c|c|c|c|c|c|c|}
\hline \multirow[b]{2}{*}{ Ensemble } & \multirow[b]{2}{*}{$n_{\text {conf }}$} & \multirow[b]{2}{*}{$n_{\text {srcs }}$} & \multirow[b]{2}{*}{$t_{\max } / a$} & \multirow[b]{2}{*}{$a \mu_{c}$} & \multicolumn{3}{|c|}{ One-state fit } & \multicolumn{3}{|c|}{ Two-state fit } & \multirow[b]{2}{*}{$a A_{\Lambda_{c}}$} & \multirow[b]{2}{*}{$B_{\Lambda_{c}} / Z_{P}$} \\
\hline & & & & & $t_{\text {low }} / a$ & $\bar{\chi}^{2}$ & $a m_{\Lambda_{c}}$ & $t_{\text {low }} / a$ & $\bar{\chi}^{2}$ & $a m_{\Lambda_{c}}$ & & \\
\hline \multirow[t]{3}{*}{ cA211.30.32 } & 287 & 16 & 22 & 0.21476 & 8 & 1.0 & $1.0200(26)$ & 3 & 1.2 & $1.0180(32)$ & $0.632(2)$ & $1.807(11)$ \\
\hline & & & & 0.26786 & 8 & 1.0 & $1.1177(29)$ & 3 & 1.1 & $1.1153(39)$ & & \\
\hline & & & & 0.32214 & 8 & 0.9 & $1.2137(34)$ & 3 & 1.0 & $1.2108(48)$ & & \\
\hline \multirow[t]{3}{*}{ cA211.12.48 } & 119 & 16 & 22 & 0.21476 & 8 & 1.0 & $1.0038(35)$ & 1 & 0.2 & $1.0011(36)$ & $0.619(4)$ & $1.792(23)$ \\
\hline & & & & 0.26786 & 8 & 0.8 & $1.1009(43)$ & 1 & 0.3 & $1.0980(43)$ & & \\
\hline & & & & 0.32214 & 8 & 0.7 & $1.1957(52)$ & 1 & 0.3 & $1.1925(52)$ & & \\
\hline \multirow[t]{3}{*}{ cB211.25.32 } & 360 & 16 & 26 & 0.17464 & 8 & 1.6 & $0.8554(18)$ & 1 & 1.3 & $0.8542(17)$ & $0.533(2)$ & $1.8454(80)$ \\
\hline & & & & 0.21830 & 8 & 1.5 & $0.9375(20)$ & 1 & 1.2 & $0.9362(18)$ & & \\
\hline & & & & 0.26196 & 8 & 1.4 & $1.0163(22)$ & 1 & 1.2 & $1.0148(20)$ & & \\
\hline \multirow[t]{3}{*}{ cB211.25.48 } & 649 & 16 & 31 & 0.17464 & 9 & 1.3 & $0.8506(12)$ & 1 & 1.2 & $0.8504(10)$ & $0.527(1)$ & $1.8520(58)$ \\
\hline & & & & 0.21830 & 9 & 1.2 & $0.9331(13)$ & 1 & 1.1 & $0.9327(12)$ & & \\
\hline & & & & 0.26196 & 9 & 1.2 & $1.0121(15)$ & 1 & 1.1 & $1.0115(13)$ & & \\
\hline \multirow[t]{2}{*}{ cB211.14.64 } & 232 & 8 & 27 & 0.20000 & 9 & 1.0 & $0.8932(25)$ & 2 & 1.2 & $0.8912(24)$ & $0.519(2)$ & $1.870(12)$ \\
\hline & & & & 0.22000 & 9 & 1.1 & $0.9306(26)$ & 2 & 1.4 & $0.9284(25)$ & & \\
\hline \multirow[t]{2}{*}{ cB211.072.64 } & 400 & 4 & 24 & 0.20000 & 11 & 1.2 & $0.8713(47)$ & 4 & 1.1 & $0.8687(57)$ & $0.501(4)$ & $1.850(23)$ \\
\hline & & & & 0.22000 & 11 & 1.2 & $0.9083(50)$ & 4 & 1.1 & $0.9057(60)$ & & \\
\hline \multirow[t]{2}{*}{ cC211.20.48 } & 205 & 4 & 29 & 0.18000 & 13 & 0.8 & $0.7869(45)$ & 4 & 0.6 & $0.7835(52)$ & $0.455(4)$ & $1.840(24)$ \\
\hline & & & & 0.22000 & 13 & 0.8 & $0.8605(50)$ & 4 & 0.6 & $0.8571(59)$ & & \\
\hline \multirow[t]{2}{*}{ cC211.06.80 } & 260 & 4 & 28 & 0.18000 & 12 & 0.9 & $0.7633(48)$ & 3 & 0.7 & $0.7614(49)$ & $0.434(4)$ & $1.825(24)$ \\
\hline & & & & 0.24000 & 12 & 1.0 & $0.8729(57)$ & 3 & 0.8 & $0.8708(57)$ & & \\
\hline
\end{tabular}

$$
\begin{aligned}
m_{N}\left(m_{u d}\right)= & m_{N}^{0}-4 c_{1}\left(2 B m_{u d}\left(1+c_{2} a^{2}\right)\right) \\
& -\frac{3 g_{A}^{2}}{16 \pi f_{\pi}^{2}}\left(2 B m_{u d}\left(1+c_{2} a^{2}\right)\right)^{3 / 2},
\end{aligned}
$$

consistent to the order we are working and including $\mathcal{O}\left(a^{2}\right)$ effects both in the pion expansion in Eq. (63) with the coefficient $c_{2}$ and in the nucleon expansion in Eq. (5). We thus have two fit parameters, $B$ and $c_{2}$, while the lattice spacings, $m_{N}^{0}$ and $c_{1}$, are determined from Eqs. (5)-(7). The fit procedure is performed for the four values of the renormalization constant from Table VII, checking for consistency and estimating systematic effects in the determination of $Z_{P}$. Since the values we obtain using the different methods of extracting $Z_{P}$ are in very good agreement, we average over them. The statistical error in

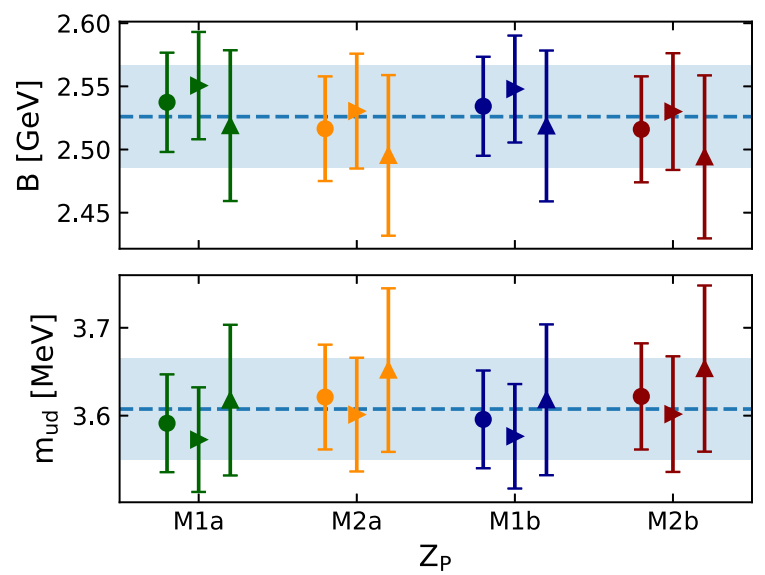

FIG. 15. Left panel: we show the nucleon mass $m_{N}$ for the A (red), B (blue), and C (green) ensembles. The blue band shows the continuum extrapolation according to Eq. (63). Right panel: the values of the parameter $B$ (top) and light quark mass (bottom) for different determinations of $Z_{P}$. Circles show the results using the mass of the nucleon from one-state fit in Table XVI. Right-pointing triangles show results when $t_{\text {low }}$ is increased by one unit, namely, $t_{\text {low }} / a+1$. Up-pointing triangles show results when the chiral extrapolation is done using ensembles with pion mass lower than $190 \mathrm{MeV}$. The dashed blue line is our final value obtained by the results listed in Table XIX. 
TABLE XIX. Fit results for the extraction of the light quark mass with the nucleon mass using the four different estimation of the renormalization constants. The fit parameters are $B$ and $c_{2}$. The light quark mass $m_{u d}$ is obtained as $m_{\pi}^{\text {isoQCD }} /(2 B)$. The values are given in the $\overline{\mathrm{MS}}$ scheme at $2 \mathrm{GeV}$.

\begin{tabular}{llllr}
\hline \hline$Z_{P}$ & $\bar{\chi}^{2}$ & $B(\mathrm{GeV})$ & $c_{2}\left(\mathrm{GeV}^{2}\right)$ & $m_{u d}(\mathrm{MeV})$ \\
\hline M1a & 0.7 & $2.537(39)$ & $0.155(98)$ & $3.591(56)$ \\
M2a & 0.7 & $2.516(41)$ & $0.49(11)$ & $3.621(60)$ \\
M1b & 0.8 & $2.534(39)$ & $0.154(98)$ & $3.596(56)$ \\
M2b & 0.7 & $2.516(42)$ & $0.57(11)$ & $3.622(60)$ \\
\hline \hline
\end{tabular}

the lattice spacing is taken into account in the jackknife analysis. The fit results are reported in Table XIX and depicted in Fig. 15. The final value of the light quark mass using the nucleon and pion mass, given in the $\overline{\mathrm{MS}}$ scheme at $2 \mathrm{GeV}$, is

$$
m_{u d}=3.608(58)\left({ }_{-19}^{+32}\right) \mathrm{MeV},
$$

obtained by averaging the values in Table XIX. The systematic error is computed as in Eq. (42), but in the sum, we only take into account the mean values not included in the computation of the average. Namely, the systematic error reflects the choice of the fitting range estimated by increasing $t_{\text {low }}$ by one unit and the sensitivity due to the chiral extrapolation estimated by using ensembles with pion mass smaller than $190 \mathrm{MeV}$. We will follow this procedure also for the computation of the systematic errors also for the strange and charm quark masses.

\section{Strange and charm quark masses}

We determine the strange and charm quark masses using the experimental value of the $\Omega(s s s)$ and $\Lambda_{c}(u d c)$ masses and the lattice spacings determined from the nucleon mass. Namely, we use $m_{\Omega}^{(\text {phys. })}=1672.5(3)$ and $m_{\Lambda_{c}}^{(\text {phys })}=$ 2286.5(1) from the particle data group (PDG) [36]. We use the renormalization constants $Z_{P}$ given in Table VII.

We parametrize the $\Omega^{-}$and $\Lambda_{c}$ mass dependence on the strange and charm quark mass by expanding around $\tilde{m}_{s}$ and $\tilde{m}_{c}$, that we chose to be in the same ballpark of the physical quark masses. In particular, we use $\tilde{m}_{s}=95 \mathrm{MeV}$ and $\tilde{m}_{c}=1.2 \mathrm{GeV}$, and we interpolate around these reference points using

$$
\begin{gathered}
m_{\Omega}=A_{\Omega}+B_{\Omega}\left(m_{s}-\tilde{m}_{s}\right), \\
m_{\Lambda_{c}}=A_{\Lambda_{c}}+B_{\Lambda_{c}}\left(m_{c}-\tilde{m}_{c}\right) .
\end{gathered}
$$

We first discuss our procedure for the determination of the strange quark mass from the $\Omega$ mass. We then apply the same procedure for determining $m_{c}$ using the $\Lambda_{c}$ mass.

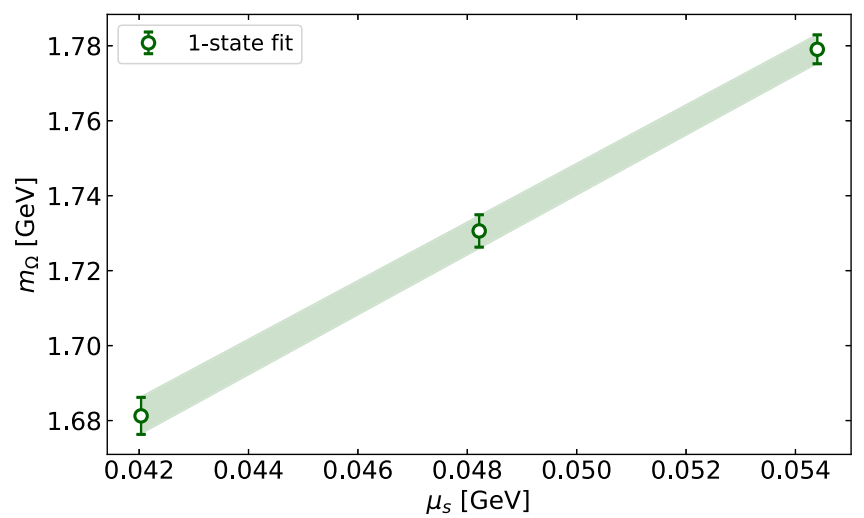

FIG. 16. Dependence of $m_{\Omega}$ on the $\mu_{s}$ bare quark mass for the cB211.072.64 ensemble. Data from the 1-state are reported, together with the linear extrapolation.

\section{Strange quark mass}

The knowledge of the $\Omega^{-}$mass at three values of the valence strange quark mass parameter $\mu_{s}$ allows us to determine the $\Omega$ mass as a function of $\mu_{s}$ using the linear Ansatz of Eq. (65). We show a representative example of the resulting fit in Fig. 16 for the ensemble cB211.072.64. The same analysis is carried out for all the ensembles listed in Table XVII, where we give the values of $A_{\Omega}$ and $B_{\Omega}$, defined in Eq. (65).

We employ two methods to determine $m_{s}$ : In method I, we perform a chiral and continuum extrapolation of the $A_{\Omega}$ and $B_{\Omega}$ parameters separately. Namely, we expand to leading order in ChPT and include $\mathcal{O}\left(a^{2}\right)$ cutoff effects as follows,

$$
\begin{aligned}
& A_{\Omega}\left(a, m_{\pi}^{2}\right)=c_{1}+c_{2} m_{\pi}^{2}+c_{3} a^{2}, \\
& B_{\Omega}\left(a, m_{\pi}^{2}\right)=c_{1}^{\prime}+c_{2}^{\prime} m_{\pi}^{2}+c_{3}^{\prime} a^{2},
\end{aligned}
$$

and we limit ourselves to ensembles with $m_{\pi}<260 \mathrm{MeV}$ so that these leading-order expression are reliable.

In Fig. 17, we illustrate the chiral and continuum extrapolation for the parameters $A_{\Omega}$ and $B_{\Omega}$ using the value of $Z_{P}$ from method M1a (see Table VII). We note that the values of $A_{\Omega}$ and $B_{\Omega}$ using the cB211.025.32 and cB211.025.48 ensembles are compatible, demonstrating that finite size effects are small. Using the values of the parameters $A_{\Omega}$ and $B_{\Omega}$ at the physical pion mass and continuum limit, we can extract the strange quark mass in the continuum limit and at the physical pion mass from

$$
m_{s}=\tilde{m}_{s}+\frac{m_{\Omega}^{(\text {phys. })}-A_{\Omega}\left(0, m_{\pi}^{(\text {phys. })}\right)}{B_{\Omega}\left(0, m_{\pi}^{(\text {phys. })}\right)} .
$$

In method II, we adopt an iterative strategy: namely, we start by fixing a value of the renormalized strange quark 

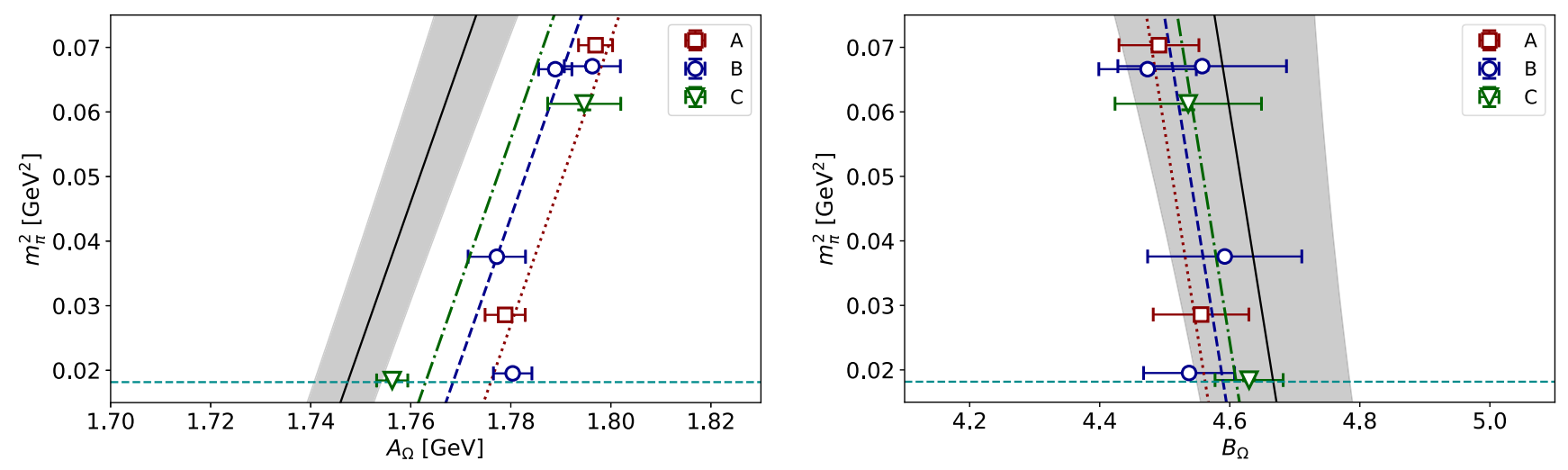

FIG. 17. Continuum and chiral extrapolation for the $\Omega^{-}$in order to determine the coefficients of Eqs. (67) and (68). We show the pion mass squared as a function of $A_{\Omega}$ (left) and $B_{\Omega}$ (right) for the A (red), B (blue), and $\mathrm{C}$ (green) ensembles. The gray bands show the continuum extrapolation.

mass $m_{s}$ in physical units for all the ensembles. We use Eq. (65) to interpolate to the given $m_{s}$. We then extrapolate to the continuum limit and physical point using the ChPT result

$$
m_{\Omega}=m_{\Omega}^{(0)}-4 c_{\Omega}^{(1)} m_{\pi}^{2}+d_{\Omega}^{(2)} a^{2}
$$

We iterate this procedure changing the value of $m_{s}$ until the resulting value of $m_{\Omega}$ given in Eq. (70) at the physical point and continuum limit matches the physical value $m_{\Omega}^{\text {(phys) }}$. In Fig. 18, we illustrate the analysis.

The results for the renormalized strange quark mass in the $\overline{\mathrm{MS}}$ scheme at $2 \mathrm{GeV}$ are provided in Table $\mathrm{XX}$ using the values of $Z_{P}$ given in Table VII. We compare the different values by plotting them in Fig. 19. As can be seen,

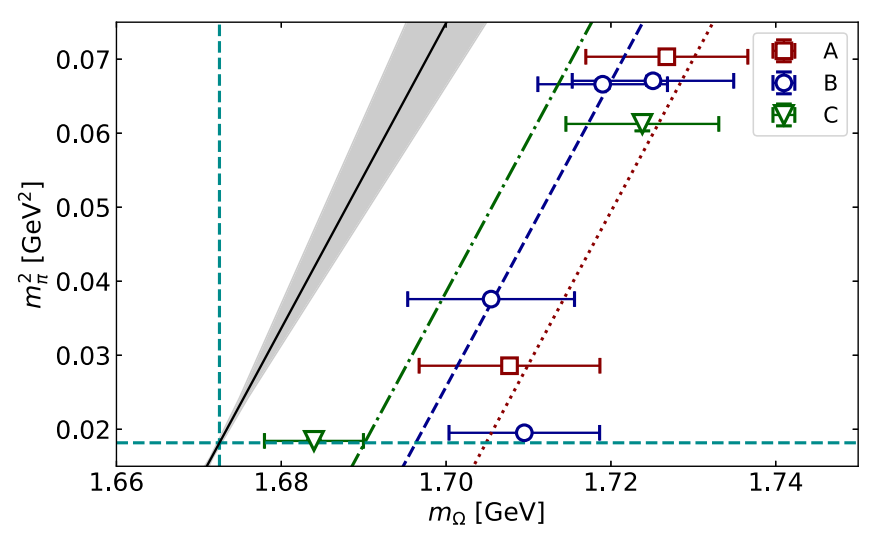

FIG. 18. We show the mass of the $\Omega^{-}, m_{\Omega}$ at different pion mass squared for $m_{s}=94.6(20) \mathrm{MeV}$, set by reproducing the physical mass of the $\Omega$ at the continuum limit as described in method II. The dotted lines show the chiral extrapolation for the A (red), B (blue), and C (green) ensembles. The solid black line shows the continuum extrapolation using Eq. (70) with the associated error (gray band). The horizontal and vertical dashed light blue lines represent, respectively, the physical pion and $\Omega$ masses. despite the different values of $Z_{P}$ at finite lattice spacing, in the continuum limit, we obtain very good agreement among different estimates of $m_{s}$. A similar agreement is also obtained between methods I and II discussed in this section for the determination of $m_{s}$. Since the error on the lattice spacing cannot be taken into account in a jackknife analysis because we used different statistics, we estimate the change in the value of $m_{s}$ by varying the lattice spacing by a standard deviation. As can be seen in Fig. 19, this gives a

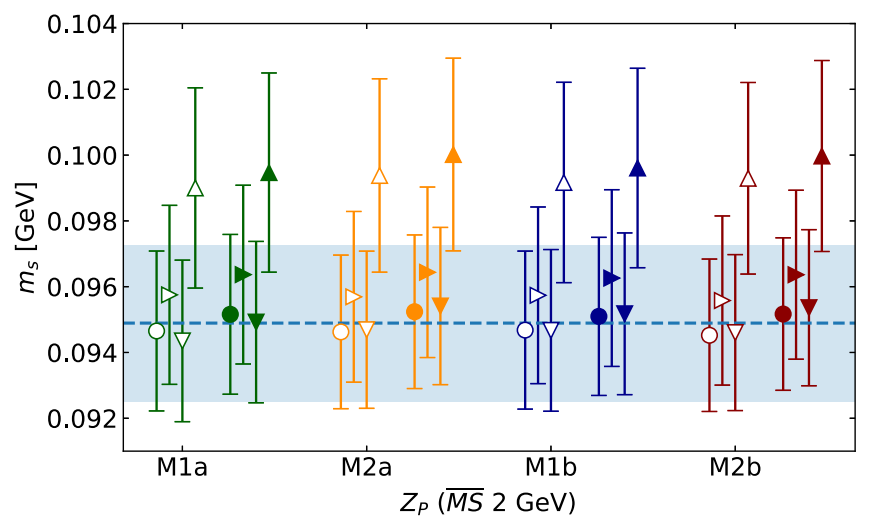

FIG. 19. The renormalized strange quark mass for different values of $Z_{P}$ in the $\overline{\mathrm{MS}}$ at $2 \mathrm{GeV}$. Symbols in green are obtained using method M1a, in orange are obtained using method M2a, in blue are obtained using method M1b, and in red are obtained using method M2b. For each value of $Z_{P}$, we give with open symbols the determination using method I to extract $m_{s}$ and with filled symbols using method II. Circles show the results using the mass of the $\Omega$ from the one-state fit given in Table XVII. Triangles quantify systematic errors due to the selection of $t_{\text {low }}$ (right-pointing triangles when $t_{\text {low }} / a+1$ ), errors on the lattice spacing (down-pointing triangles increasing by a standards deviation the lattice spacings set by the nucleon mass), and errors due to chiral extrapolation (up-pointing triangles obtained using only ensembles with $m_{\pi}<190 \mathrm{MeV}$ ). The dashed line is the average over the values from method I and II and at different $Z_{P}$ given in Tables $\mathrm{XX}$ and $\mathrm{XXI}$. 
TABLE XX. Results using method I and different values of $Z_{P}$ as denoted in the first column. The second, third, fourth, and fifth columns give the reduced $\chi^{2}$ of the fit to Eq. (67) and the values of the fit parameters $c_{1}, c_{2}$, and $c_{3}$, respectively, that determine $A_{\Omega}$ in the continuum and chiral limit. The sixth, seventh, eight, and ninth columns give the corresponding values for $B_{\Omega}$ of Eq. (68). In the last column, we give the extracted values for $m_{s}$ in the $\overline{\mathrm{MS}}$ scheme at $2 \mathrm{GeV}$.

\begin{tabular}{|c|c|c|c|c|c|c|c|c|c|}
\hline \multirow[b]{2}{*}{$Z_{P}$} & \multicolumn{4}{|c|}{$A_{\Omega}$} & \multicolumn{4}{|c|}{$B_{\Omega}$} & \multirow{2}{*}{$\frac{\overline{\mathrm{MS}}(2 \mathrm{GeV})}{m_{s}(\mathrm{MeV})}$} \\
\hline & $\bar{\chi}$ & $c_{1}(\mathrm{GeV})$ & $c_{2}\left(\mathrm{GeV}^{-2}\right)$ & $c_{3}\left(\mathrm{GeV} \mathrm{\textrm {fm } ^ { - 2 } )}\right.$ & $\bar{\chi}^{\prime}$ & $c_{1}^{\prime}$ & $c_{2}^{\prime}\left(\mathrm{GeV}^{-2}\right)$ & $c_{3}^{\prime}\left(\mathrm{fm}^{-2}\right)$ & \\
\hline M1a & 3.7 & $1.739(7)$ & $0.45(7)$ & $3.3(1.1)$ & 0.2 & $4.69(11)$ & $-1.5(1.3)$ & $-12(19)$ & $94.6(2.5)$ \\
\hline $\mathrm{M} 2 \mathrm{a}$ & 4.4 & $1.739(6)$ & $0.44(6)$ & $5.45(97)$ & 0.2 & $4.67(12)$ & $-1.6(1.4)$ & $23(20)$ & $94.6(2.3)$ \\
\hline M1b & 3.2 & $1.739(6)$ & $0.44(7)$ & $2.3(1.0)$ & 0.3 & $4.68(11)$ & $-1.6(1.3)$ & $-10(19)$ & $94.6(2.4)$ \\
\hline $\mathrm{M} 2 \mathrm{~b}$ & 4.6 & $1.739(6)$ & $0.44(6)$ & $6.19(96)$ & 0.2 & $4.67(12)$ & $-1.6(1.4)$ & $31(20)$ & $94.5(2.3)$ \\
\hline
\end{tabular}

TABLE XXI. Results using method II and different values of $Z_{P}$ as denoted in the first column. In the second column, we give the reduced $\chi^{2}$ of the fit, and in columns three, four, and five, we give the fit parameters of Eq. (70). In the last column, we give the extracted values for $m_{s}$ in the $\overline{\mathrm{MS}}$ scheme at $2 \mathrm{GeV}$.

\begin{tabular}{llllll}
\hline \hline$Z_{P}$ & $\bar{\chi}$ & $m_{\Omega}^{(0)}(\mathrm{GeV})$ & $c_{\Omega}^{(1)}\left(\mathrm{GeV}^{-1}\right)$ & $d_{\Omega}^{(2)}\left(\mathrm{GeV} \mathrm{fm}^{-2}\right)$ & $\overline{\mathrm{MS}(2 \mathrm{GeV})}$ \\
\hline M1a & 2.4 & $1.6636(16)$ & $-0.121(21)$ & $3.7(1.3)$ & $m_{s}(\mathrm{MeV})$ \\
M2a & 2.7 & $1.6637(15)$ & $-0.120(20)$ & $5.3(1.2)$ & $95.1(2.5)$ \\
M1b & 2.2 & $1.6638(15)$ & $-0.118(21)$ & $2.6(1.2)$ & $95.2(2.3)$ \\
M2b & 2.7 & $1.6637(15)$ & $-0.120(20)$ & $6.0(1.2)$ & $95.0(2.4)$ \\
\hline \hline
\end{tabular}

very small change compared to the statistical error. By increasing $t_{\text {low }}$ of the one-state fit by one lattice unit gives an estimate of the systematic error in the extraction of the mass of the $\Omega$. The change in the value of $m_{s}$ is well within the statistical error, as can be seen in Fig. 19. We, thus, average over all the values obtained using the four different determinations of $Z_{P}$ and analysis methods I and II (values are given in Tables XX an Table XXI). The systematic error is computed according to Eq. (42) but excluding the values over which we average. As for the case of $\mu_{u d}$, the systematic error reflects systematics due to the choice of the fitting range by letting $t_{\text {low }} / a \rightarrow t_{\text {low }} / a+1$ and systematics due to the chiral extrapolation by using ensembles with $m_{\pi}<190 \mathrm{MeV}$. Since the error in the lattice spacing cannot be included in the jackknife analysis due to using different statistics for the $\Omega$, we include an additional term in Eq. (42) computed as the difference in the mean when we change the lattice spacing within its error. Using as input the $\Omega^{-}$mass, we obtain for $m_{s}$ in the $\overline{\mathrm{MS}}$ scheme at $2 \mathrm{GeV}$ and the ratio $m_{s} / m_{u d}$ the values

$m_{s}=94.9(2.4)\left(\begin{array}{c}+4.1 \\ -1.0\end{array}\right) \mathrm{MeV}, \quad m_{s} / m_{u d}=26.30(61)\left(\begin{array}{l}+1.17 \\ -33\end{array}\right)$

where for the ratio we use $m_{u d}$ from Eq. (64). The error on the ratio is computed by combining in quadrature the errors on $m_{u d}$ and $m_{s}$.

\section{Charm quark mass}

We employ the same procedure for the determination of $m_{c}$ as described in the previous section for $m_{s}$ using methods I and II. The mass of $\Lambda_{c}$ is interpolated linearly in $\mu_{c}$ using Eq. (66) within the range spanned by the two $\mu_{c}$

TABLE XXII. The same as in Table XX but for the $A_{\Lambda_{c}}$ and $B_{\Lambda_{c}}$ parameters. In the last two columns, the values for $m_{c}$ in the $\overline{\mathrm{MS}}$ scheme at $3 \mathrm{GeV}$ are reported.

\begin{tabular}{|c|c|c|c|c|c|c|c|c|c|}
\hline \multirow[b]{2}{*}{$Z_{P}$} & \multicolumn{4}{|c|}{$A_{\Lambda_{c}}$} & \multicolumn{4}{|c|}{$B_{\Lambda_{c}}$} & \multirow{2}{*}{$\frac{\overline{\mathrm{MS}}(3 \mathrm{GeV})}{m_{c}(\mathrm{MeV})}$} \\
\hline & $\bar{\chi}$ & $c_{1}(\mathrm{GeV})$ & $c_{2}\left(\mathrm{GeV}^{-1}\right)$ & $c_{3}\left(\mathrm{GeV} \mathrm{fm}{ }^{-2}\right)$ & $\bar{\chi}^{\prime}$ & $c_{1}^{\prime}$ & $c_{2}^{\prime}\left(\mathrm{GeV}^{-2}\right)$ & $c_{3}^{\prime}\left(\mathrm{fm}^{-2}\right)$ & \\
\hline M1a & 2.1 & $2.55(2)$ & $0.9(2)$ & $-4.9(2.6)$ & 1.3 & $1.14(2)$ & $0.00(15)$ & $-13.7(2.5)$ & $1030(21)$ \\
\hline M2a & 2.1 & $2.55(2)$ & $0.9(2)$ & $3.6(2.7)$ & 1.6 & $1.15(2)$ & $0.01(16)$ & $-6.4(2.6)$ & $1027(20)$ \\
\hline M1b & 2.2 & $2.54(2)$ & $0.9(2)$ & $-3.8(2.6)$ & 1.0 & $1.14(2)$ & $-0.03(15)$ & $-12.8(2.5)$ & $1037(21)$ \\
\hline $\mathrm{M} 2 \mathrm{~b}$ & 2.1 & $2.55(2)$ & $1.0(2)$ & $5.5(2.8)$ & 1.7 & $1.15(2)$ & $0.01(16)$ & $-4.8(2.6)$ & $1025(20)$ \\
\hline
\end{tabular}



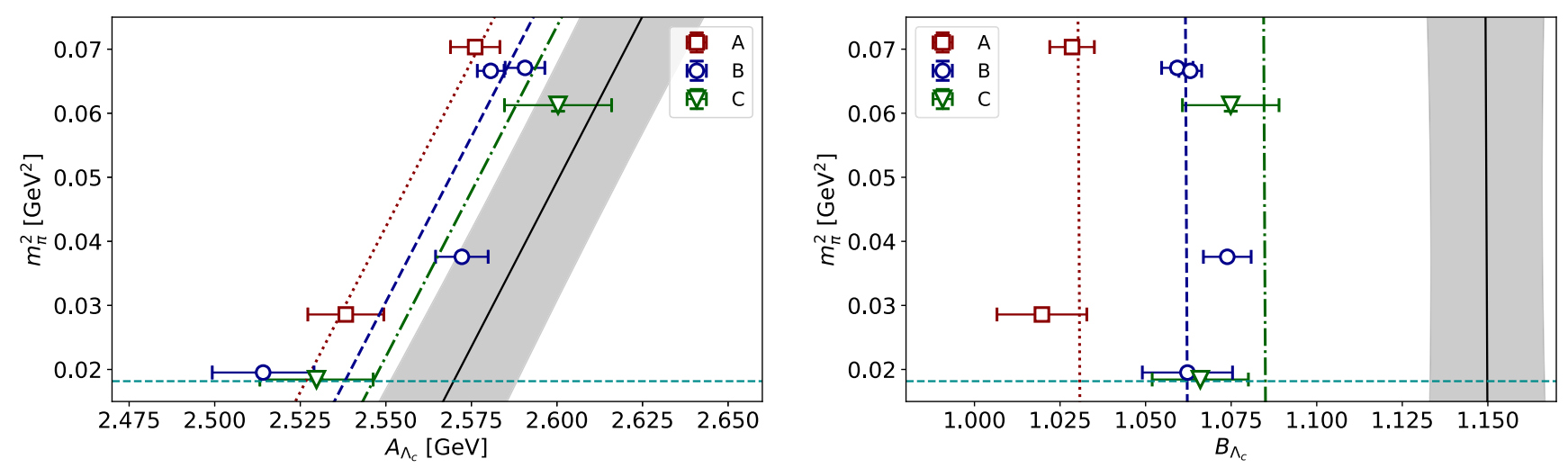

FIG. 20. The same as for Fig. 17 but for the case of $\Lambda_{c}$.

values. For the chiral extrapolation, we consider a similar expression to that used for $\Omega^{-}$as given in Eq. (70), namely,

$$
m_{\Lambda_{c}}=m_{\Lambda_{c}}^{(0)}+c_{\Lambda_{c}}^{(1)} m_{\pi}^{2}+d_{\Lambda_{c}}^{(2)} a^{2}
$$

We note that to leading one loop in ChPT a $m_{\pi}^{3}$-term with an unknown coefficient is present. Including such a term in the fit results in a coefficient consistent with zero and a $\chi^{2} /$ d.o.f. $=7.2$. That such a term is not supported by lattice QCD data was also found in our previous analysis using a larger set of pion masses [19] where this coefficient was found to be consistent with zero. We check that including it does not change the extracted value for $m_{c}$. Thus, given the larger $\chi^{2}$, we drop it from our analysis.

Results from method I are reported in Table XXII for all the values of $Z_{P}$ listed in Table VII. We also illustrate in Fig. 20 the chiral and continuum extrapolations of $A_{\Lambda_{c}}$ and $B_{\Lambda_{c}}$ according to Eqs. (67) and (68), respectively, with $Z_{P}$ determined using method M1a. The determination of the values of the parameters of Eq. (72) for method II is carried out as for the case of $m_{s}$, and the results are reported in Table XXIII and, for the M1a case, also in Figs. 21 and 22.

The values for $m_{c}$ from methods I and II as well as how they change by varying the lattice spacings by a standard deviation and by the change in $m_{\Lambda_{c}}$ by increasing $t_{\text {low }}$ by one lattice spacing in the one-state fit are presented in Fig. 21.

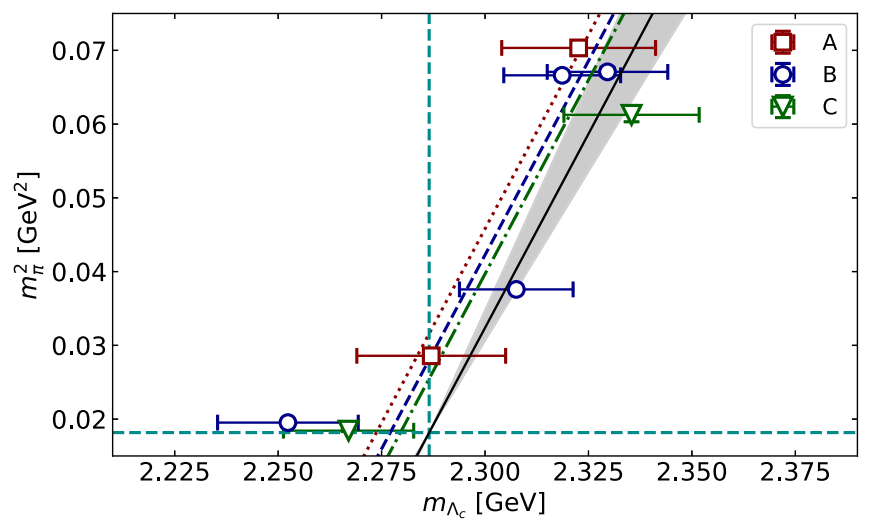

FIG. 21. The same as for Fig. 18 but for the case of $\Lambda_{c}$.

Again, we observe a very good agreement between the results obtained via method I and method II and among different determinations of the $Z_{P}$ renormalization constants. We thus average over these values and compute the systematic error in the same way as for $\mu_{s}$. We obtain for the charm quark mass $m_{c}$ in the $\overline{\mathrm{MS}}$ scheme at $3 \mathrm{GeV}$ and the ratio $m_{c} / m_{s}$ the following values,

$m_{c}=1030(21)\left({ }_{-5}^{+22}\right) \mathrm{MeV}, \quad m_{c} / m_{s}=12.05(31)\left({ }_{-15}^{+58}\right)$,

where the errors on the ratio are combined in quadrature.

TABLE XXIII. The same as in Table XXI but for the $m_{\Lambda_{c}}$ extrapolation, according to Eq. (72). In the last column, we give the values for $m_{c}$ in the $\overline{\mathrm{MS}}$ scheme at $3 \mathrm{GeV}$.

\begin{tabular}{lcccrc}
\hline \hline & & & & & \\
$Z_{P}$ & $\bar{\chi}$ & $m_{\Lambda_{c}}^{(0)}(\mathrm{GeV})$ & $c_{\Lambda_{c}}^{(1)}\left(\mathrm{GeV}^{-1}\right)$ & $d_{\Lambda_{c}}^{(2)}\left(\mathrm{GeV} \mathrm{fm}^{-2}\right)$ & $\overline{\mathrm{MS}(3 \mathrm{GeV})}$ \\
\hline M1a & 2.7 & $2.2692(25)$ & $0.95(14)$ & $-1.4(2.0)$ & $1030(21)$ \\
M2a & 2.7 & $2.2689(26)$ & $0.96(14)$ & $5.3(2.2)$ & $1026(20)$ \\
M1b & 2.8 & $2.2695(25)$ & $0.93(14)$ & $-0.6(2.0)$ & $1038(21)$ \\
M2b & 2.7 & $2.2688(26)$ & $0.97(15)$ & $6.7(2.2)$ & $1024(20)$ \\
\hline \hline
\end{tabular}


TABLE XXIV. The renormalized quark masses determined in the meson sector (first row) and baryon sector (second row) in the $\overline{\mathrm{MS}}$ scheme. In the third row, we give the average over the values obtained in the meson and baryon sectors, while in the last row, we give the latest FLAG averages [13] for $N_{f}=2+1+1$. The light quark mass, $m_{u d}$ (second column), and the strange quark mass, $m_{s}$ (third column), are given at $2 \mathrm{GeV}$, while the charm quark mass, $m_{c}$ (fourth column), is given at $3 \mathrm{GeV}$. The second error of the quark masses includes a $0.5 \%$ uncertainty (added in quadrature) due to the uncertainty of the conversion of the renormalization constants (RCs) $Z_{P}$ to the $\overline{\mathrm{MS}}$ scheme. In the fifth and sixth columns, we give the ratios $m_{s} / m_{u d}$ and $m_{c} / m_{s}$, respectively. In the meson sector, the error on the ratio is determined in a jackknife analysis. In the baryon sector, since different ensembles are involved in the determination of the quark masses, the error on the ratio is propagated quadratically using the errors on each of the quark masses.

\begin{tabular}{lccccc}
\hline \hline & $m_{u d}(\mathrm{MeV})$ & $m_{s}(\mathrm{MeV})$ & $m_{c}(\mathrm{MeV})$ & $m_{s} / m_{u d}$ & $m_{c} / m_{s}$ \\
\hline Meson sector & $3.689(80)(66)$ & $101.0(1.9)(1.4)$ & $1039(15)(8)$ & $27.30(24)(14)$ & $11.43(9)(10)$ \\
Baryon sector & $3.608(58)\left(\begin{array}{c}+32 \\
-19\end{array}\right)$ & $94.9(2.4)\left(\begin{array}{c}+4.1 \\
-1.0\end{array}\right)$ & $1030(21)\left(\begin{array}{c}+22 \\
-5\end{array}\right)$ & $26.30(61)\left(\begin{array}{c}+1.17 \\
-0.33\end{array}\right)$ & $12.04(31)\left(\begin{array}{c}+58 \\
-15\end{array}\right)$ \\
Average & $3.636(66)\left(\begin{array}{l}+60 \\
-57\end{array}\right)$ & $98.7(2.4)\left({ }_{-3.2}^{+4.0}\right)$ & $1036(17)\left({ }_{-8}^{+15}\right)$ & $27.17(32)\left({ }_{-38}^{+56}\right)$ & $11.48(12)\left(\begin{array}{c}+25 \\
-19\end{array}\right)$ \\
FLAG 2019 & $3.410(43)$ & $93.44(68)$ & $988(7)$ & $27.23(10)$ & $11.82(16)$ \\
\hline \hline
\end{tabular}

\section{CONCLUSIONS}

The focus of this work is the determination of the light, strange, and charm quark masses. We perform an analysis of ten $N_{f}=2+1+1$ ensembles simulated at three lattice spacings smaller than $0.1 \mathrm{fm}$ and pion masses in the range from about 350 to $135 \mathrm{MeV}$. Having two ensembles simulated with the physical value of the pion mass at the two smallest lattice spacings enables us to extrapolate reliably to the physical and continuum limit.

The extraction of the quark masses is done using observables from both the meson sector and the baryon sector. The isosymmetric values of the pion, kaon, and $D$ meson masses as well as of the pion decay constant are used for the determination of the lattice spacings and the quark masses in the meson analysis. In the baryon sector, we use as inputs the nucleon and pion masses to obtain the lattice spacing and the average light-quark mass, while the masses of the $\Omega^{-}$and $\Lambda_{c}$ baryons determine the strange and charm quark masses.

In Table XXIV, we collect the values of the quark masses obtained in Secs. V and VI for the light and strange quark masses in the $\overline{\mathrm{MS}}$ scheme at $2 \mathrm{GeV}$ and for the charm quark mass at $3 \mathrm{GeV}$. Since the isospin and electromagnetic corrections to the nucleon mass are only known for the mass difference between the neutron and proton [37], in our analysis, we average over the mass of the proton and neutron. This defines a QCD prescription different from that used in the meson sector. Using the values of the lattice spacing extracted in the meson sector, we obtain a nucleon mass in the continuum limit a few MeV smaller than the input value $m_{N, p h y s}=0.9389 \mathrm{GeV}$ adopted in the baryon sector (see Sec. III). This results in less than a percent change in the values given in the Table XXIV, which is much smaller than our statistical errors. It is thus justifiable to average over the values obtained in the meson and baryon sectors to produce our final values.
In order to perform the above average, we adopt the weighted approach given in Eqs. (40)-(42). We assume the following weights,

$$
w_{M} \propto 1 /\left(\sigma_{M}^{\text {stat }}\right)^{2}, \quad w_{B} \propto 1 /\left(\sigma_{B}^{\text {stat }}\right)^{2},
$$

for the quantities coming from the mesonic and the baryonic sectors, where $\sigma_{M(B)}^{\text {stat }}$ is the first error given in the corresponding rows of Table XXIV. In this way, we obtain

$$
\bar{x} \pm \sigma^{\mathrm{stat}}\left(\left(_{-\sigma^{\mathrm{yss},-},-}^{\mathrm{syst},+},\right.\right.
$$

where

$$
\begin{gathered}
\bar{x}=w_{M} x_{M}+w_{B} x_{B}, \\
\left(\sigma^{\text {stat }}\right)^{2}=w_{M}\left(\sigma_{M}^{\text {stat }}\right)^{2}+w_{B}\left(\sigma_{B}^{\text {stat }}\right)^{2},
\end{gathered}
$$

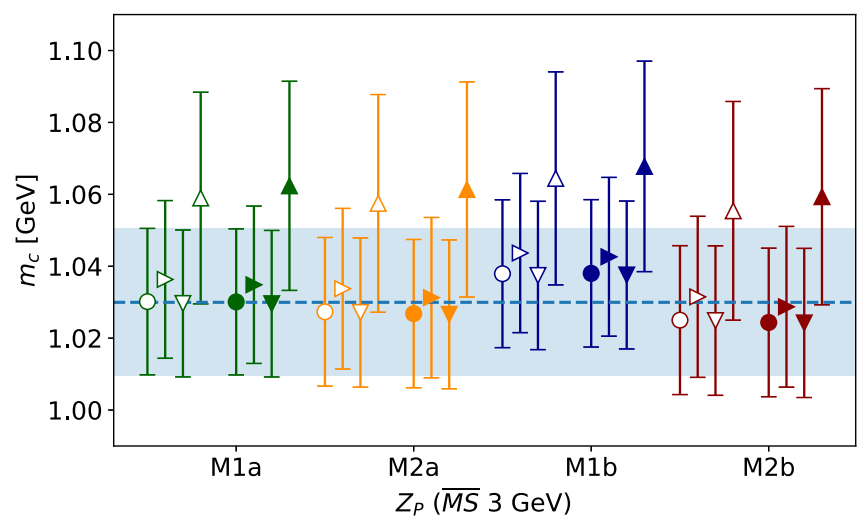

FIG. 22. We show the values of $m_{c}$ for different determinations of $Z_{P}$ and for methods I and II. The notation is the same as that of Fig. 19. 

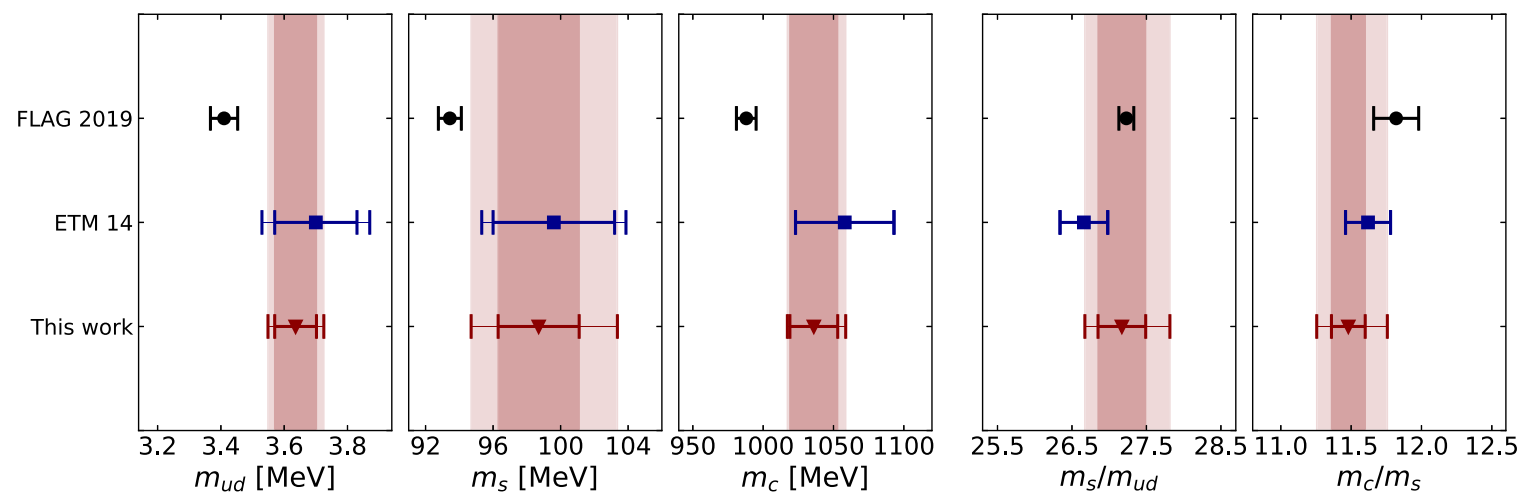

FIG. 23. Comparison of the results average between the values determined in the meson and baryon sectors (red triangles) with the values obtained using twisted-mass fermions in Ref. [12] (blue squares) and the $N_{f}=2+1+1$ averages given in the last FLAG report [13] (black circles). The shorter error bars take into account the statistical error only, while the larger represent the total error, obtained by summing in quadrature the statistical and the systematic errors.

$$
\begin{aligned}
\left(\sigma^{\text {syst } \pm}\right)^{2}= & w_{M}\left[\left(x_{M}-\bar{x}\right)^{2}+\left(\sigma_{M}^{\text {syst }}\right)^{2}\right] \\
& +w_{B}\left[\left(x_{B}-\bar{x}\right)^{2}+\left(\sigma_{B}^{\text {syst } \pm}\right)^{2}\right] .
\end{aligned}
$$

The results are given in the last row of Table XXIV and are compared in Fig. 23 with those of the ETM analysis of Ref. [12] and the ones entering the $N_{f}=2+1+1$ averages in the latest FLAG report [13]. The latter ones are based on the results of Refs. [12,38] for the light-quark mass, Refs. [12,38-40] for the strange mass, Refs. [12,19,3840] for the charm mass, Refs. [12,41,42] for the $m_{s} / m_{u d}$ ratio, and Refs. [12,38,40] for the $m_{c} / m_{s}$ ratio.

It can be seen that our results are larger by approximately 2.5 standard deviations in the case of $m_{u d}$ and by approximately 2 standard deviations in the case of $m_{c}$ with respect to the corresponding FLAG values. Although for the strange quark mass our result coming from the meson sector is larger by approximately 3 standard deviations, our averaged result is consistent with the FLAG one within our final uncertainty. Good agreement is observed for the mass ratios $m_{s} / m_{u d}$ and $m_{c} / m_{s}$. We do not believe that these differences can be ascribed to possible uncontrolled effects on the mass renormalization constant $1 / Z_{P}$. Indeed, the detailed analysis carried out in this work concerning the pion pole subtraction and the residual hadronic contaminations in the RI-MOM determination of the renormalization constant $Z_{P}$ leaves little room for any significant leftover contribution from these terms. Our findings point to the fact that hadronic contaminations are controlled at the level of few per mille. Therefore, we do not consider it plausible that the observed tension with the FLAG values may be related to uncontrolled hadronic contaminations on the mass renormalization constant. In this respect, we are considering the possibility of repeating the determination of the quark masses using the same extended twisted mass (ETM) gauge ensembles adopted in this work, but evaluating the mass renormalization in a different scheme, like
RI-SMOM, while keeping the same level of control of the hadronic contaminations achieved in this work.

Our final results for the light, strange, and charm quark masses as well for the mass ratios $m_{s} / m_{u d}$ and $m_{c} / m_{s}$ are consistent with our previous analysis of Ref. [12] (see also Fig. 23), which was based on Wilson twisted-mass fermions far from the physical pion point. The overall uncertainties for the light and charm quark masses are reduced by a factor of approximately $1.7-2.0$, while in the case of the strange quark mass, the uncertainty is almost unchanged, partly due to the difference between the mean values obtained in the meson and baryon sector, which is added to the systematic error. This is also reflected in the two ratios. With respect to the quark mass analysis of Ref. [12], the main improvements are (i) a better control of the chiral extrapolation thanks to gauge ensembles produced close to the physical pion point; (ii) a better control of hadronic contaminations in the calculations of the mass renormalization constant; and (iii) the use of both mesonic and baryonic quantities, which requires simulations of different correlation functions. For all the three masses, the contribution from lattice systematics is important, in particular in the case of $m_{s}$. Our plan is to add at least one further gauge ensemble at a fourth finer value of the lattice spacing at the physical point. This will allow a tightly controlled chiral and continuum extrapolations in both the meson and baryon sectors.

\section{ACKNOWLEDGMENTS}

We would like to thank all members of ETMC for a very constructive and enjoyable collaboration. We acknowledge Partnership for Advanced Computing in Europe (PRACE) for awarding us access to the highperformance computing system Marconi and Marconi100 at Consorzio Interuniversitario per il Calcolo Automatico dell'Italia Nord-orientale under the Grants No. Pra17-4394, No. Pra20-5171, and No. Pra22-5171 and CINECA for 
providing us CPU time under the specific initiative INFNLQCD123. We also acknowledge PRACE for awarding us access to HAWK, hosted by HLRS, Germany, under the grant with Acid 33037. The authors gratefully acknowledge the Gauss Centre for Supercomputing e.V. (www.gausscentre.eu) for funding the project pr74yo by providing computing time on the GCS Supercomputer SuperMUC at Leibniz Supercomputing Centre (www.lrz.de); the projects ECY00, HCH02, and HBN28 on the GCS supercomputers JUWELS and JUWELS Booster [43] at the Jülich Supercomputing Centre (JSC); and time granted by the John von Neumann Institute for Computing on the supercomputers JURECA and JURECA Booster [44], also at JSC. Part of the results were created within the EA program of JUWELS Booster also with the help of the JUWELS Booster Project Team (JSC, Atos, ParTec, NVIDIA). We further acknowledge computing time granted on Piz Daint at Centro Svizzero di Calcolo Scientifico via Project No. s702. Part of the statistics of the cA211.30.32 ensemble used in this work was generated on the Bonna cluster at the University of Bonn, access to which the authors gratefully acknowledge. This work has been partially supported by the Horizon 2020 research and innovation program of the European Commission under the Marie Skłodowska-Curie Grant No. 765048 (STIMULATE) as well as by the DFG as a project under the Sino-German CRC110. R. F. acknowledges the University of Rome Tor Vergata for the support granted to the project PLNUGAMMA. F. S. and S. S. are supported by the Italian Ministry of Research under Grant No. PRIN 20172LNEEZ. F. S. is supported by INFN under GRANT73/CALAT. P. D. and E. F. acknowledge support form the European Unions Horizon 2020 research and innovation programme under the Marie Skłodowska-Curie Grant No. 813942 (EuroPLEx). P. D. acknowledges support from INFN under the research project INFN-QCDLAT. M.C. acknowledges financial support by the U.S. Department of Energy, Office of Nuclear Physics Early Career Award under Grant No. DE-SC0020405. S. B. and J. F. are supported by the $\mathrm{H} 2020$ project PRACE 6-IP (Grant No. 82376) and the EuroCC project (Grant No. 951740). F. M. and A. T. are supported by the European Joint Doctorate program STIMULATE Grant No. 765048. K. H. and E. P. are supported by the Cyprus Research and Innovation Foundation under Contract No. POST-DOC/0718/0100. F. P. acknowledges support from project NextQCD, cofunded by the European Regional Development Fund and the Republic of Cyprus through the Research and Innovation Foundation (EXCELLENCE/0918/0129). M. D. C. is supported in part by UK STFC Grant No. ST/ P000630/1.
[1] R. Frezzotti, P. A. Grassi, S. Sint, and P. Weisz (Alpha Collaboration), J. High Energy Phys. 08 (2001) 058.

[2] B. Sheikholeslami and R. Wohlert, Nucl. Phys. B259, 572 (1985).

[3] R. Baron et al., J. High Energy Phys. 06 (2010) 111.

[4] R. Frezzotti and G. C. Rossi, J. High Energy Phys. 08 (2004) 007.

[5] R. Frezzotti and G. C. Rossi, J. High Energy Phys. 10 (2004) 070.

[6] M. Constantinou et al. (ETM Collaboration), J. High Energy Phys. 08 (2010) 068.

[7] R. Frezzotti, G. Martinelli, M. Papinutto, and G. C. Rossi, J. High Energy Phys. 04 (2006) 038.

[8] P. Boucaud et al. (ETM Collaboration), Comput. Phys. Commun. 179, 695 (2008).

[9] A. Abdel-Rehim et al. (ETM Collaboration), Phys. Rev. D 95, 094515 (2017).

[10] C. Alexandrou et al., Phys. Rev. D 98, 054518 (2018).

[11] C. Alexandrou et al., arXiv:2104.06747.

[12] N. Carrasco et al. (European Twisted Mass Collaboration), Nucl. Phys. B887, 19 (2014).

[13] S. Aoki et al. (Flavour Lattice Averaging Group), Eur. Phys. J. C 80, 113 (2020).

[14] R. Frezzotti and G. C. Rossi, Nucl. Phys. B, Proc. Suppl. 128, 193 (2004).
[15] R. Baron et al. (European Twisted Mass Collaboration), Comput. Phys. Commun. 182, 299 (2011).

[16] K. Osterwalder and E. Seiler, Ann. Phys. (N.Y.) 110, 440 (1978).

[17] S. Aoki et al., Eur. Phys. J. C 77, 112 (2017).

[18] C. Alexandrou and C. Kallidonis, Phys. Rev. D 96, 034511 (2017).

[19] C. Alexandrou, V. Drach, K. Jansen, C. Kallidonis, and G. Koutsou, Phys. Rev. D 90, 074501 (2014).

[20] J. Gasser, M. E. Sainio, and A. Svarc, Nucl. Phys. B307, 779 (1988).

[21] B. C. Tiburzi and A. Walker-Loud, Phys. Lett. B 669, 246 (2008).

[22] B. Märkisch et al., Phys. Rev. Lett. 122, 242501 (2019).

[23] G. Martinelli, C. Pittori, C. T. Sachrajda, M. Testa, and A. Vladikas, Nucl. Phys. B445, 81 (1995).

[24] M. Constantinou, R. Horsley, H. Panagopoulos, H. Perlt, P. E. L. Rakow, G. Schierholz, A. Schiller, and J. M. Zanotti, Phys. Rev. D 91, 014502 (2015).

[25] C. Alexandrou, M. Constantinou, and H. Panagopoulos (ETM Collaboration), Phys. Rev. D 95, 034505 (2017).

[26] K. G. Chetyrkin and A. Retey, Nucl. Phys. B583, 3 (2000).

[27] S. R. Sharpe, Phys. Rev. D 56, 7052 (1997); 62, 099901(E) (2000).

[28] S. Borsanyi et al., J. High Energy Phys. 09 (2012) 010. 
[29] M. Lüscher, J. High Energy Phys. 08 (2010) 071; 03 (2014) 092(E).

[30] G. Bergner, P. Dimopoulos, J. Finkenrath, E. Fiorenza, R. Frezzotti, M. Garofalo, B. Kostrzewa, F. Sanfilippo, S. Simula, and U. Wenger (Extended Twisted Mass Collaboration), Proc. Sci. LATTICE2019 (2020) 181 [arXiv:2001.09116].

[31] J. Gasser and H. Leutwyler, Ann. Phys. (N.Y.) 158, 142 (1984).

[32] B. Blossier, M. Della Morte, G. von Hippel, T. Mendes, and R. Sommer, J. High Energy Phys. 04 (2009) 094.

[33] C. R. Allton et al. (UKQCD Collaboration), Phys. Rev. D 47, 5128 (1993).

[34] M. Falcioni, M. Paciello, G. Parisi, and B. Taglienti, Nucl. Phys. B251, 624 (1985).

[35] O. Bar, Phys. Rev. D 92, 074504 (2015).

[36] P. D. Group, P. A. Zyla et al., Prog. Theor. Exp. Phys. 2020, 083C01 (2020).
[37] S. Borsanyi et al., Science 347, 1452 (2015).

[38] A. Bazavov et al. (Fermilab Lattice, MILC, TUMQCD Collaborations), Phys. Rev. D 98, 054517 (2018).

[39] A. T. Lytle, C. T. H. Davies, D. Hatton, G. P. Lepage, and C. Sturm (HPQCD Collaboration), Phys. Rev. D 98, 014513 (2018).

[40] B. Chakraborty, C. T. H. Davies, B. Galloway, P. Knecht, J. Koponen, G. C. Donald, R. J. Dowdall, G. P. Lepage, and C. McNeile, Phys. Rev. D 91, 054508 (2015).

[41] A. Bazavov et al. (Fermilab Lattice, MILC Collaborations), Phys. Rev. D 90, 074509 (2014).

[42] A. Bazavov et al., Phys. Rev. D 98, 074512 (2018).

[43] Jülich Supercomputing Centre, J. Large-Scale Res. Facil. 5, A135 (2019).

[44] Jülich Supercomputing Centre, J. Large-Scale Res. Facil. 4 (2018). 